\title{
Differential graded bocses and $A_{\infty}$-modules
}

\author{
R. Bautista, E. Pérez and L. Salmerón
}

June 25, 2019

Dedicated to J.A. De la Peña on the occasion of his 60th birthday

\begin{abstract}
We introduce and study the category of twisted modules over a triangular differential graded bocs. We show that in this category idempotents split, that it admits a natural structure of a Frobenius category, that a twisted module is homotopically trivial iff its underlying complex is acyclic, and that any homotopy equivalence of differential graded bocses determines an equivalence of the corresponding homotopy categories of twisted modules. The category of modules over an $A_{\infty}$-algebra is equivalent to the category of twisted modules over a triangular differential graded bocs, so all the preceding statements lift to the former category.
\end{abstract}

\section{Introduction}

There is some analogy between the theory of modules over bocses and the theory of $A_{\infty}$-modules over an $A_{\infty}$-algebra. This can be noticed, for instance, in the fact that the morphisms $f: M \longrightarrow N$ of modules over a given triangular bocs can be handled as pairs $\left(f^{0}, f^{1}\right)$ of morphisms, where the properties of the first component $f^{0}: M \longrightarrow N$ reflect on the properties of the whole morphism $f$, similarly, the properties of a morphism $g: M \longrightarrow N$ of $A_{\infty}$-modules over a given $A_{\infty}$-algebra depend on the properties of the first component $g_{1}: M \longrightarrow N$.

For instance, the natural exact structure on the category of modules of a given triangular bocs is determined by the first components of morphisms, while in the category of modules over a given $A_{\infty}$-algebra the natural exact structure is determined by the first components of morphisms.

2010 Mathematics Subject Classification: Primary 06B15, 16T15, 18E30; Secondary $16 \mathrm{G} 20$.

Key words and phrases: differential graded bocses, differential graded coalgebras, $A_{\infty^{-}}$ algebras, $A_{\infty}$-modules, Frobenius category, twisted graded modules. 
Motivated by this analogy, we work here with the natural notions of differential graded bocses and their category of twisted graded modules, as defined below in (2.1) and (2.5). The study of these algebraic structures led us to simpler proofs of some well known facts on categories of $A_{\infty}$-modules.

In order to describe more precisely our constribution, let us fix some notation and recall some well known concepts. Throughout this article, we denote by $k$ a fixed ground field, which will act centrally on any $k$-algebra and on any $k$ $k$-bimodule we consider. We also fix a finite-dimensional semisimple $k$-algebra $S$. Moreover, we ask that $S \otimes_{k} S^{o p}$ is also semisimple. This holds, for instance when $k$ is a perfect field or if $S$ is a finite product of copies of the ground field. We will make explicit any further requirement on $S$, when needed. We consider $S$ as a $\mathbb{Z}$-graded $k$-algebra concentrated at degree 0 .

We will consider graded right $S$-modules (or graded left $S$-modules, or graded $S$-S-bimodules) $M$, so $M$ is equipped with a direct sum decomposition

$$
M=\bigoplus_{j \in \mathbb{Z}} M_{j}
$$

of right $S$-submodules $M_{j}$ (resp. left $S$-submodules, or $S$-S $S$-subbimodules). The elements $x \in M_{j}$ are called homogeneous of degree $j$, and we indicate this fact by $|x|=j$. Given two graded right $S$-modules, a homogeneous morphism $f: M \longrightarrow N$ of degree $|f|=d$ satisfies that $f\left(M_{j}\right) \subseteq N_{j+d}$, for any $j \in \mathbb{Z}$. We denote by $\operatorname{Hom}_{\mathrm{GMod}-S}^{d}(M, N)$ the space of homogeneous morphisms of graded right $S$-modules $f: M \longrightarrow N$ of degree $d$. Then, we have the graded category GMod- $S$ of graded right $S$-modules with hom spaces

$$
\operatorname{Hom}_{\mathrm{GMod}-S}(M, N)=\bigoplus_{d \in \mathbb{Z}} \operatorname{Hom}_{\mathrm{GMod}-S}^{d}(M, N) .
$$

Similarly, we have the graded category GMod- $S$ - $S$ of graded $S$-S-bimodules.

The tensor product $M \otimes_{S} N$ of two graded objects $M$ and $N$ is equipped with the standard grading given by the homogeneous components

$$
\left(M \otimes_{S} N\right)_{j}=\bigoplus_{s+t=j} M_{s} \otimes_{S} N_{t} .
$$

Assume that $f: M \longrightarrow N$ is a homogeneous morphism of graded right $S$-modules and $g: M^{\prime} \longrightarrow N^{\prime}$ is a homogeneous morphism of graded left $S$ modules. Then, their tensor product $f \otimes g$ is the homogeneous linear map $M \otimes_{S} M^{\prime} \longrightarrow N \otimes_{S} N^{\prime}$ of degree $|f \otimes g|=|f|+|g|$ defined, for any homogeneous elements $x \in M$ and $y \in M^{\prime}$, by the following formula.

$$
[f \otimes g](x \otimes y):=(-1)^{|g||x|} f(x) \otimes g(y) .
$$

So, if $f: M \longrightarrow N, h: N \longrightarrow L$ are morphisms of graded right $S$-modules, and $g: M^{\prime} \longrightarrow N^{\prime}, t: N^{\prime} \longrightarrow L^{\prime}$ are morphisms of graded left $S$-modules, we have

$$
(h \otimes t)(f \otimes g)=(-1)^{|t||f|} h f \otimes t g .
$$

Now we recall some basic definitions of the theory of $A_{\infty}$-algebras. 
Definition 1.1. An $A_{\infty}$-algebra $A$ is a graded $S$-S-bimodule $A$, equipped with a sequence of homogeneous morphisms of $S$-S-bimodules

$$
\left\{m_{n}: A^{\otimes n} \longrightarrow A\right\}_{n \in \mathbb{N}}
$$

where each $m_{n}$ has degree $\left|m_{n}\right|=2-n$, such that, for each $n \in \mathbb{N}$, the following Stasheff identity holds.

$$
S_{n}: \sum_{\substack{r+s+t=n \\ s \geq 1 ; r, t \geq 0}}(-1)^{r+s t} m_{r+1+t}\left(i d^{\otimes r} \otimes m_{s} \otimes i d^{\otimes t}\right)=0 .
$$

Given two $A_{\infty}$-algebras $\left(A,\left\{m_{n}\right\}\right)$ and $\left(B,\left\{m_{n}^{\prime}\right\}\right)$, a morphism of $A_{\infty}$-algebras $f:\left(A,\left\{m_{n}\right\}\right) \longrightarrow\left(B,\left\{m_{n}^{\prime}\right\}\right)$ is a family of homogeneous $S$-S-bimodule morphisms $\left\{f_{n}: A^{\otimes n} \longrightarrow B\right\}_{n \in \mathbb{N}}$ such that each $f_{n}$ is homogeneous of degree $\left|f_{n}\right|=1-n$ and the equality $\Sigma_{n}=\Sigma_{n}^{\prime}$ holds for all $n \in \mathbb{N}$, where

$$
\begin{aligned}
\Sigma_{n}= & \sum_{\substack{r+s+t=n \\
r, t \geq 0 ; s \geq 1}}(-1)^{r+s t} f_{r+1+t}\left(i d^{\otimes r} \otimes m_{s} \otimes i d^{\otimes t}\right), \\
\Sigma_{n}^{\prime}= & \sum_{\substack{1 \leq r \leq n \\
i_{1}+\cdots+i_{r}=n}}(-1)^{\operatorname{sgn}\left(i_{1}, \ldots, i_{r}\right)} m_{r}^{\prime}\left(f_{i_{1}} \otimes \cdots \otimes f_{i_{r}}\right),
\end{aligned}
$$

where $i_{1}, \ldots, i_{r} \geq 1$ and

$\operatorname{sgn}\left(i_{1}, \ldots, i_{r}\right)=(r-1)\left(i_{1}-1\right)+(r-2)\left(i_{2}-1\right)+\cdots+2\left(i_{r-2}-1\right)+\left(i_{r-1}-1\right)$.

Given two morphisms of $A_{\infty}$-algebras $f: A \longrightarrow B$ and $g: B \longrightarrow C$, their composition $g \circ f: A \longrightarrow C$ is the family $(g \circ f)=\left\{(g \circ f)_{n}\right\}_{n \in \mathbb{N}}$ defined, for each $n \in \mathbb{N}$, by

$$
(g \circ f)_{n}=\sum_{\substack{1 \leq s \leq n \\ i_{1}+\cdots+i_{s}=n}}(-1)^{\operatorname{sgn}\left(i_{1}, \ldots, i_{s}\right)} g_{s}\left(f_{i_{1}} \otimes \cdots \otimes f_{i_{s}}\right) .
$$

The preceding notions give rise to a $k$-category $\mathrm{Alg}_{\infty}$ of $A_{\infty}$-algebras with morphisms of $A_{\infty}$-algebras. There is a well known full and faithful functor $\Psi: \operatorname{Alg}_{\infty} \longrightarrow$ DGCoalg, where DGCoalg denotes the category of differential graded $S$-coalgebras, which has been used successfully to study the category $\mathrm{Alg}_{\infty}$, see [6], 7] and their references. The functor $\Psi$ is given by the bar construction: it maps each $A_{\infty}$-algebra $\left(A,\left\{m_{n}\right\}\right)$ onto its reduced tensor $S$ coalgebra $\bar{T}_{S}(A[1])$, equipped with a differential $\delta$ induced by the family $\left\{m_{n}\right\}$, see $[\underline{6}(3.6)$.

There is some notational difference with [6] and [7, which is a minor one as explained by the following. 
Lemma 1.2. Let $A$ be a graded $S$-S-bimodule equipped with a sequence of homogeneous morphisms of $S$-S-bimodules $\left\{m_{n}: A^{\otimes n} \longrightarrow A\right\}_{n \in \mathbb{N}}$. For $n \in \mathbb{N}$, define

$$
m_{n}^{\prime}:=(-1)^{\frac{n(n-1)}{2}} m_{n}
$$

Then, the equation $S_{n}$ appearing in the last definition holds iff the following equation holds

$$
S_{n}^{\prime}: \sum_{\substack{r+s+t=n \\ s \geq 1 ; r, t \geq 0}}(-1)^{r s+t} m_{r+t+1}^{\prime}\left(i d^{\otimes r} \otimes m_{s} \otimes i d^{\otimes t}\right)=0 .
$$

Moreover, if we make

$$
Z_{n}=\sum_{\substack{r+s+t=n \\ s \geq 1 ; r, t \geq 0}}(-1)^{r+s t} m_{r+t+1}\left(i d^{\otimes r} \otimes m_{s} \otimes i d^{\otimes t}\right)
$$

and

$$
Z_{n}^{\prime}=\sum_{\substack{r+s+t=n \\ s \geq 1 ; r, t \geq 0}}(-1)^{r s+t} m_{r+t+1}^{\prime}\left(i d^{\otimes r} \otimes m_{s}^{\prime} \otimes i d^{\otimes t}\right),
$$

we have that

$$
Z_{n}^{\prime}=(-1)^{\frac{n(n-1)}{2}} Z_{n}
$$

Proof. By definition,

$$
Z_{n}^{\prime}=\sum_{\substack{r+s+t=n \\ s \geq 1 ; r, t \geq 0}}(-1)^{r s+t}(-1)^{\frac{(r+t+1)(r+t)}{2}}(-1)^{\frac{s(s-1)}{2}} m_{r+t+1}\left(i d^{\otimes r} \otimes m_{s} \otimes i d^{\otimes t}\right) .
$$

The non-negative integers $r, s, t$ satisfy $r+s+t=n$ and $s \geq 1$. Then, our statement follows from the next congruence modulo 2

$$
r s+t+\frac{(r+t+1)(r+t)}{2}+\frac{s(s-1)}{2} \equiv r+s t+\frac{n(n-1)}{2} .
$$

Thus, the choice for the signs in the formulas we made in the last definition, which is also adopted by other authors, is not essential. It will reflect on some signs in the formulas involving $A_{\infty}$-objects.

Definition 1.3. Let $A=\left(A,\left\{m_{n}\right\}\right)$ be an $A_{\infty}$-algebra. Then, a graded right $S$-module $M$ is called a right $A_{\infty}$-module over $A$ iff $M$ is equipped with a family $\left\{m_{n}^{M}\right\}_{n \in \mathbb{N}}$ of morphism of right $S$-modules such that, each map

$$
m_{n}^{M}: M \otimes A^{\otimes(n-1)} \longrightarrow M
$$


is homogeneous of degree $\left|m_{n}^{M}\right|=2-n$ and, for every $n \in \mathbb{N}$, the condition $\Sigma_{n}^{+}+\Sigma_{n}^{0}=0$ is satisfied, where

$$
\Sigma_{n}^{+}=\sum_{\substack{r+s+t=n \\ t \geq 0 ; r, s \geq 1}}(-1)^{r+s t} m_{r+1+t}^{M}\left(i d^{\otimes r} \otimes m_{s} \otimes i d^{\otimes t}\right)
$$

and

$$
\Sigma_{n}^{0}=\sum_{\substack{s+t=n \\ t \geq 0 ; s \geq 1}}(-1)^{s t} m_{1+t}^{M}\left(m_{s}^{M} \otimes i d^{\otimes t}\right) .
$$

In the sum $\Sigma_{n}^{+}$, for simplicity, we abuse of the language writing $i d^{\otimes r}$ instead of $i d_{M} \otimes i d_{A}^{\otimes(r-1)}$. In case $n=1$, the condition reduces to $\Sigma_{1}^{0}=0$ which is equivalent to say that $m_{1}^{M}$ satisfies $\left(m_{1}^{M}\right)^{2}=0$.

Given $M=\left(M,\left\{m_{n}^{M}\right\}\right)$ and $N=\left(N,\left\{m_{n}^{N}\right\}\right)$ right $A_{\infty}$-modules over $A$. Then, a morphism of right $A_{\infty}$-modules $f: M \longrightarrow N$ over $A$ is a family $f=$ $\left\{f_{n}\right\}_{n \in \mathbb{N}}$, where each

$$
f_{n}: M \otimes_{S} A^{\otimes(n-1)} \longrightarrow N
$$

is a homogeneous morphism of graded right $S$-modules of degree $\left|f_{n}\right|=1-n$ such that, for each $n \in \mathbb{N}$, the equality $\Sigma_{n}^{f+}+\Sigma_{n}^{f 0}+\Sigma_{n}^{f-}=0$, where

$$
\begin{gathered}
\Sigma_{n}^{f+}=\sum_{\substack{r+s+t=n \\
t \geq 0 ; r, s \geq 1}}(-1)^{r+s t} f_{r+1+t}\left(i d^{\otimes r} \otimes m_{s} \otimes i d^{\otimes t}\right), \\
\Sigma_{n}^{f 0}=\sum_{\substack{s+t=n \\
t \geq 0 ; s \geq 1}}(-1)^{s t} f_{1+t}\left(m_{s}^{M} \otimes i d^{\otimes t}\right)
\end{gathered}
$$

and

$$
\Sigma_{n}^{f-}=-\sum_{\substack{r+s=n \\ r \geq 1 ; s \geq 0}}(-1)^{(r+1) s} m_{1+s}^{N}\left(f_{r} \otimes i d^{\otimes s}\right) .
$$

The condition in case $n=1$ is equivalent to $m_{1}^{N} f_{1}=f_{1} m_{1}^{M}$, that is, to the requirement that the map $f_{1}: M \longrightarrow N$ is a morphism of complexes of right $S$-modules. The morphism $f$ is called a quasi-isomorphism iff $f_{1}$ is so.

The class of right $A_{\infty}$-modules together with the morphisms between them is a category with the following composition. If $f: M \longrightarrow N$ and $g: N \longrightarrow L$ are morphisms of right $A_{\infty}$-modules over $A$, their composition

$$
g \circ f=\left\{(g \circ f)_{n}\right\}_{n \in \mathbb{N}}: M \longrightarrow L
$$

is defined, for each $n \in \mathbb{N}$, by

$$
(g \circ f)_{n}=\sum_{\substack{r+s=n \\ r \geq 1 ; s \geq 0}}(-1)^{(r+1) s} g_{1+s}\left(f_{r} \otimes i d^{\otimes s}\right) .
$$

Given a left $A_{\infty}$-module $M$, the identity $\mathbb{I}_{M}=\left\{h_{n}\right\}: M \longrightarrow M$ is given by $h_{1}=i d_{M}$ and $h_{n}=0$, for all $n \geq 2$. We shall denote this category by $\operatorname{Mod}_{\infty}-A$. 
For the study of the category $\operatorname{Mod}_{\infty}-A$ one of the main tools is the graded category DGComod- $\mathcal{B}_{A}$, the category of differential graded comodules over the differential tensor $S$-coalgebra $\mathcal{B}_{A}=\left(T_{S}(A[1]), \mu, \epsilon, \delta\right)$, with $\mu$ the comultiplication given by the bar construction, $\epsilon: T_{S}(A[1]) \longrightarrow S$ is the canonical projection, and $\delta$ is the differential induced by the family of operations $\left\{m_{n}\right\}_{n \in \mathbb{N}}$ of the $A_{\infty}$-algebra $A$. This is so, because of there is a full and faithful functor $\Phi: \operatorname{Mod}_{\infty}-A \longrightarrow \mathrm{DGComod}^{0}-\mathcal{B}_{A}$. The superindex 0 indicates the subcategory of DGComod- $\mathcal{B}_{A}$ with the same objects but with only degree zero homogeneous morphisms.

Finally, we recall the appropriate notions of homotopy for the preceding categories $\operatorname{Alg}_{\infty}$ and $\operatorname{Mod}_{\infty}-A$.

Definition 1.4. Let $A$ be an $A_{\infty}$-algebra and $f, g: M \longrightarrow N$ morphisms of right $A_{\infty}$-modules over $A$. Then, a homotopy from $f$ to $g$ is a family of maps $\left\{h_{n}\right\}_{n \in \mathbb{N}}$ where, for each $n \in \mathbb{N}$,

$$
h_{n}: M \otimes_{S} A^{\otimes(n-1)} \longrightarrow N
$$

is a homogeneous morphism of right $S$-modules of degree $\left|h_{n}\right|=-n$, such that, for all $n \in \mathbb{N}$, we have $f_{n}-g_{n}=H_{n}^{(1)}+H_{n}^{(2)}+H_{n}^{(3)}$, where

$$
\begin{aligned}
& H_{n}^{(1)}=\sum_{\substack{r+s=n \\
r \geq 1 ; s \geq 0}}(-1)^{r s} m_{1+s}^{N}\left(h_{r} \otimes i d^{\otimes s}\right), \\
& H_{n}^{(2)}=\sum_{\substack{s+t=n \\
s \geq 1 ; t \geq 0}}(-1)^{s t} h_{1+t}\left(m_{s}^{M} \otimes i d^{\otimes t}\right),
\end{aligned}
$$

and

$$
H_{n}^{(3)}=\sum_{\substack{r+s+t=n \\ r, s \geq 1 ; t \geq 0}}(-1)^{r+s t} h_{r+1+t}\left(i d^{\otimes r} \otimes m_{s} \otimes i d^{\otimes t}\right),
$$

A morphism of $A_{\infty}$-modules $f: M \longrightarrow N$ is called null-homotopic iff there is a homotopy from $f$ to 0 .

Definition 1.5. Let $A$ and $B$ be $A_{\infty}$-algebras and let $f, g: A \longrightarrow B$ be morphisms of $A_{\infty}$-algebras. A homotopy $h$ from $f$ to $g$ is a family $h=\left\{h_{n}\right\}_{n \in \mathbb{N}}$, where each

$$
h_{n}: A^{\otimes n} \longrightarrow B
$$

is a homogeneous morphism of $S$-S-bimodules with degree $\left|h_{n}\right|=-n$, such that, for each $n \in \mathbb{N}$, we have

$$
f_{n}-g_{n}=H(h)_{n}+H_{f, g}(h)_{n},
$$

where

$$
H(h)_{n}=\sum_{\substack{r+s+t=n \\ r, t \geq 0 ; s \geq 1}}(-1)^{r+s t} h_{r+1+t}\left(i d^{\otimes r} \otimes m_{s}^{A} \otimes i d^{\otimes t}\right)
$$


and

$$
H_{f, g}(h)_{n}=\sum_{\begin{array}{c}
r, t \geq 0 ; s \geq 1 \\
i_{1}+\cdots+i_{r}+s \\
+j_{1}+\cdots+j_{t}=n
\end{array}}(-1)^{\mathrm{sgn}} m_{r+1+t}^{B}\left(f_{i_{1}} \otimes \cdots \otimes f_{i_{r}} \otimes h_{s} \otimes g_{j_{1}} \otimes \cdots \otimes g_{j_{t}}\right)
$$

where $i_{1}, \ldots, i_{r}, j_{1}, \ldots, j_{t} \geq 1$ and $\operatorname{sgn}=\operatorname{sgn}\left(i_{1}, \ldots, i_{r}, s, j_{1}, \ldots, j_{t}\right)$ is given by the sum

$$
r(t+1)+s t+\sum_{\substack{2 \leq \alpha \leq r \\ 1 \leq u<\alpha}}\left(1-i_{u}\right)+t \sum_{u=1}^{r} i_{u}+\sum_{\substack{2 \leq \beta \leq t \\ 1 \leq v<\beta}}\left(1-j_{v}\right) .
$$

The preceding notions of homotopy are known to be equivalence relations on the corresponding categories and give rise to the homotopy categories $\underline{\operatorname{Mod}}_{\infty}-A$ and $\underline{\mathrm{Alg}}_{\infty}$, respectively.

The categories DGCoalg and DGComod ${ }^{0}-\mathcal{B}_{A}$ have their own classical homotopy relations. The functors

$$
\Psi: \operatorname{Alg}_{\infty} \longrightarrow \text { DGCoalg and } \Phi: \operatorname{Mod}_{\infty}-A \longrightarrow \mathrm{DGComod}^{0}-\mathcal{B}_{A}
$$

preserve and reflect the preceding homotopy relations and, therefore, induce full and faithful functors $\underline{\Psi}: \underline{\mathrm{Alg}}_{\infty} \longrightarrow \underline{\mathrm{DGCoalg}}$ and $\underline{\Phi}: \underline{\operatorname{Mod}}_{\infty}-A \longrightarrow \underline{\mathrm{DGComod}}^{0}-\mathcal{B}_{A}$.

Many important properties of $\overline{\mathrm{Alg}}_{\infty}$ and $\underline{\operatorname{Mod}}_{\infty}-A$ are derived, using the full and faithful functors $\Psi$ and $\Phi$, from the corresponding properties for DGCoalg and $\underline{\mathrm{DGComod}}^{0}-\mathcal{B}_{A}$ which are better understood categories.

In this paper, we propose that the use of the categories of twisted modules over differential graded $S$-bocses can be a fresh and simpler tool for some studies of $\operatorname{Mod}_{\infty}-A$. We illustrate this with three applications.

Namely, we can describe with quite good precision the structure of $\operatorname{Mod}_{\infty}-A$ as a Frobenius category. In particular, we prove that idempotents split in $\operatorname{Mod}_{\infty}-A$, a fact which seems to have remained unnoticed. We obtain this as an application of the study of the category TGMod- $\mathcal{B}$, for a general triangular differential graded $S$-bocs, see (7.16). By "general" we mean that it is not necessarily a differential graded tensor $S$-coalgebra. We compare our description with the one given in 6] (Proposition 5.2 and 8.4).

The important fact that each quasi-isomorphism of $A_{\infty}$-modules is a homotopy equivalence, see [6] (Theorem 4.2), is obtained here, for the case where $S$ is a finite product of copies of the ground field $k$, as a consequence of our theorem (8.3). The latter is proved by an induction argument using the triangularity of the given triangular differential graded $S$-bocs. The proof does not resort to any model theoretical considerations.

Finally, we show the fact that any homotopy equivalence $f: A \longrightarrow B$ of $A_{\infty}$-algebras determines a restriction functor $R_{f}: \underline{\operatorname{Mod}}_{\infty}-A \longrightarrow \underline{\operatorname{Mod}}_{\infty}-A$ which is an equivalence of categories, see 6] (Proposition 6.2). We obtain this as a consequence of the corresponding result for triangular differential graded $S$ bocses, see (5.5), which essentially relies on the remarkable property that a 
morphism $f: M \longrightarrow N$ in the category of modules over a triangular differential graded $S$-bocs is an isomorphism if and only if its first component is so, see (4.8).

The twisted modules we consider in (2.5) are constructed from the differential graded category GMod- $\mathcal{B}$, of graded modules over a differential graded $S$-bocs $\mathcal{B}$. These twisted modules are a special kind of the twisted complexes over a general differential graded category considered in [3]. Here we focus on a naive and very concrete approach to the study of the category TGMod- $\mathcal{B}$ for a triangular differential graded $S$-bocs $\mathcal{B}$ and extract from this the preceding applications to the study of $A_{\infty}$-modules.

\section{Differential graded bocses, twisted modules}

In this paper, we use the word bocs in the following specific sense.

Definition 2.1. A graded $S$-bocs $\mathcal{B}$ is a triple $\mathcal{B}=(C, \mu, \epsilon)$, where $C$ is a graded $S$-S-bimodule, so we have a decomposition $C=\bigoplus_{j \in \mathbb{Z}} C_{j}$ as a direct sum of $S$-S-bimodules, and $\mu: C \longrightarrow C \otimes_{S} C$ and $\epsilon: C \longrightarrow S$ are homogeneous $S$-S-bimodule maps of degree 0 such that the following diagrams commute

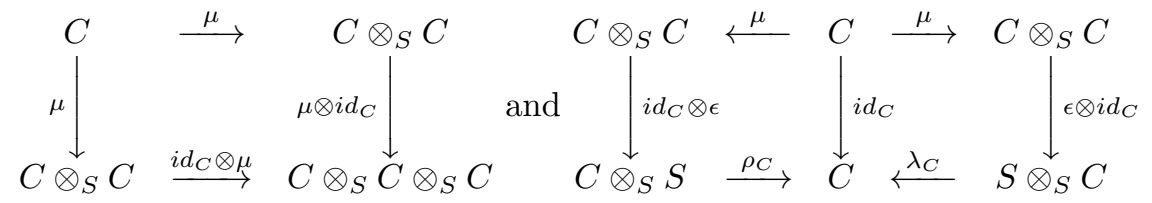

where $\lambda_{C}$ and $\rho_{C}$ are the left and right $S$-multiplications on $C$, respectively. The $S$-S-bimodule $S$ is considered as a graded $S$-bimodule concentrated at 0 .

A coderivation $\delta$ on $\mathcal{B}$ is a homogeneous morphism of $S$ - $S$-bimodules $\delta$ : $C \longrightarrow C$, of degree 1 , such that the following square commutes.

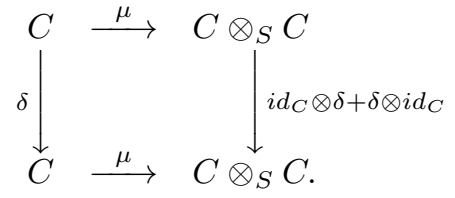

A coderivation $\delta$ on $\mathcal{B}$ is called a differential iff furthermore $\delta^{2}=0$. In this case, $\mathcal{B}=(C, \mu, \epsilon, \delta)$ is called a differential graded $S$-bocs. A morphism $f:(C, \mu, \epsilon, \delta) \longrightarrow\left(C^{\prime}, \mu^{\prime}, \epsilon^{\prime}, \delta^{\prime}\right)$ of differential graded $S$-bocses is a homogeneous morphism $f: C \longrightarrow C^{\prime}$ of graded $S$-S-bimodules of degree 0 such that $\mu^{\prime} f=(f \otimes f) \mu, \epsilon^{\prime} f=\epsilon$ and $\delta^{\prime} f=f \delta$.

So a (differential) graded $S$-bocs is exactly the same concept as a (differential) graded $S$-coalgebra. A morphism of (differential) graded $S$-bocses is the same as a morphism of (differential) graded $S$-coalgebras.

The following useful property is well known, but we include a proof for the sake of the reader. 
Lemma 2.2. For any differential graded $S$-bocs $\mathcal{B}=(C, \mu, \epsilon, \delta)$ we have $\epsilon \delta=0$.

Proof. We know that $\rho\left(i d_{C} \otimes \epsilon\right) \mu=i d_{C}$ and $\lambda\left(\epsilon \otimes i d_{C}\right) \mu=i d_{C}$. Thus, for $c \in C$, if $\mu(c)=\sum_{i} c_{i} \otimes c_{i}^{\prime}$, we get

$$
\sum_{i} \epsilon\left(c_{i}\right) c_{i}^{\prime}=c=\sum_{i} c_{i} \epsilon\left(c_{i}^{\prime}\right)
$$

Since $\delta$ is a coderivation, we have $\left[\left(i d_{C} \otimes \delta\right)+\left(\delta \otimes i d_{C}\right)\right] \mu=\mu \delta$. As a consequence, $\rho\left(i d_{C} \otimes \epsilon\right)\left(i d_{C} \otimes \delta\right) \mu+\rho\left(i d_{C} \otimes \epsilon\right)\left(\delta \otimes i d_{C}\right) \mu=\rho\left(i d_{C} \otimes \epsilon\right) \mu \delta=\delta$. Hence,

$$
\rho\left(i d_{C} \otimes \epsilon \delta\right) \mu+\rho(\delta \otimes \epsilon) \mu=\delta .
$$

Evaluate at the element $c$ to obtain $\sum_{i}(-1)^{\left|c_{i}\right|} c_{i} \epsilon \delta\left(c_{i}^{\prime}\right)+\sum_{i} \delta\left(c_{i}\right) \epsilon\left(c_{i}^{\prime}\right)=\delta(c)$. Since we also have

$$
\delta(c)=\delta\left(\sum_{i} c_{i} \epsilon\left(c_{i}^{\prime}\right)\right)=\sum_{i} \delta\left(c_{i}\right) \epsilon\left(c_{i}^{\prime}\right),
$$

we know that $\sum_{i}(-1)^{\left|c_{i}\right|} c_{i} \epsilon \delta\left(c_{i}^{\prime}\right)=0$. Applying the morphism $\epsilon$, we have $\sum_{i}(-1)^{\left|c_{i}\right|} \epsilon\left(c_{i}\right) \epsilon \delta\left(c_{i}^{\prime}\right)=0$. Since $\epsilon: C \longrightarrow S$ is homogeneous of degree 0 , we have $\epsilon\left(c_{i}\right)=0$ whenever $\left|c_{i}\right| \neq 0$, so we finally get

$$
\epsilon \delta(c)=\epsilon \delta\left(\sum_{i} \epsilon\left(c_{i}\right) c_{i}^{\prime}\right)=\sum_{i} \epsilon\left(c_{i}\right) \epsilon \delta\left(c_{i}^{\prime}\right)=0 .
$$

Definition 2.3. Given a graded $S$-bocs $\mathcal{B}=(C, \mu, \epsilon)$, we can consider the $k$-category of graded right $\mathcal{B}$-modules denoted by GMod- $\mathcal{B}$. A graded right $\mathcal{B}$ module is by definition a graded right $S$-module. Given graded $\mathcal{B}$-modules $M$ and $N$, and $d \in \mathbb{Z}$, a morphism $f: M \longrightarrow N$ of right $\mathcal{B}$-modules of degree $d$ is a homogeneous morphism of right $S$-modules $f: M \otimes_{S} C \longrightarrow N$ of degree $d$. So, their set of morphisms in the category GMod- $\mathcal{B}$ is by definition

$$
\operatorname{Hom}_{\mathrm{GMod}-\mathcal{B}}(M, N):=\bigoplus_{d \in \mathbb{Z}} \operatorname{Hom}_{\mathrm{GMod}-\mathcal{B}}^{d}(M, N)=\bigoplus_{d \in \mathbb{Z}} \operatorname{Hom}_{\mathrm{GMod}-S}^{d}\left(M \otimes_{S} C, N\right) .
$$

If we have a pair $f: M \longrightarrow N, g: N \longrightarrow L$ of composable morphisms in GMod- $\mathcal{B}$, their composition in GMod- $\mathcal{B}$ is defined as the following composition of morphisms of right $S$-modules

$$
g * f=\left(M \otimes_{S} C \stackrel{i d_{M} \otimes \mu}{\longrightarrow} M \otimes_{S} C \otimes_{S} C \stackrel{f \otimes i d_{C}}{\longrightarrow} N \otimes_{S} C \stackrel{g}{\longrightarrow} L\right) .
$$

It is not hard to show that GMod- $\mathcal{B}$ is indeed a graded $k$-category (the unit morphism at a graded $\mathcal{B}$-module $M$, denoted by $\mathbb{I}_{M}$, is the morphism of right $S$-modules $\rho_{M}\left(i d_{M} \otimes \epsilon\right)$, where $\rho_{M}: M \otimes_{S} S \longrightarrow M$ is the right multiplication on $M$. We will denote by GMod $^{0}-\mathcal{B}$ the subcategory of GMod- $\mathcal{B}$ with the same objects but only the degree zero morphisms of GMod- $\mathcal{B}$. 
Proposition 2.4. Let $\mathcal{B}=(C, \mu, \epsilon, \delta)$ be a differential graded $S$-bocs. Given $M, N \in$ GMod- $\mathcal{B}$, define on the graded hom space

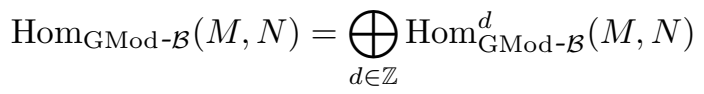

the homogeneous linear map $\hat{\delta}: \operatorname{Hom}_{\mathrm{GMod}-\mathcal{B}}(M, N) \longrightarrow \operatorname{Hom}_{\mathrm{GMod}-\mathcal{B}}(M, N)$ of degree 1 by the following recipe, for any homogeneous morphism $f: M \longrightarrow N$,

$$
\hat{\delta}(f)=(-1)^{|f|+1} f\left(i d_{M} \otimes \delta\right) .
$$

Then, the category GMod- $B$ is a differential graded category. Namely, $\hat{\delta}^{2}=0$, $\hat{\delta}\left(\mathbb{I}_{M}\right)=0$, for all $M \in$ GMod- $\mathcal{B}$, and the following Leibniz rule holds

$$
\hat{\delta}(g * f)=\hat{\delta}(g) * f+(-1)^{|g|} g * \hat{\delta}(f),
$$

for any homogeneous morphisms $f: M \longrightarrow N$ and $g: N \longrightarrow L$ in GMod-B .

Proof. For this proof make $\underline{\hat{\delta}}(f)=f\left(i d_{M} \otimes \delta\right)$. Then, we have

$$
\begin{aligned}
\hat{\hat{\delta}}(g * f)= & (g * f)\left(i d_{M} \otimes \delta\right) \\
= & g\left(f \otimes i d_{C}\right)\left(i d_{M} \otimes \mu\right)\left(i d_{M} \otimes \delta\right) \\
= & g\left(f \otimes i d_{C}\right)\left(i d_{M} \otimes \mu \delta\right) \\
= & g\left(f \otimes i d_{C}\right)\left(i d_{M} \otimes\left[\delta \otimes i d_{C}+i d_{C} \otimes \delta\right] \mu\right) \\
= & g\left(f \otimes i d_{C}\right)\left(i d_{M} \otimes \delta \otimes i d_{C}\right)\left(i d_{M} \otimes \mu\right) \\
& +g\left(f \otimes i d_{C}\right)\left(i d_{M} \otimes i d_{C} \otimes \delta\right)\left(i d_{M} \otimes \mu\right) \\
= & g\left(f\left(i d_{M} \otimes \delta\right) \otimes i d_{C}\right)\left(i d_{M} \otimes \mu\right) \\
& +g\left(f\left(i d_{M} \otimes i d_{C}\right) \otimes \delta\right)\left(i d_{M} \otimes \mu\right) \\
= & g\left(f\left(i d_{M} \otimes \delta\right) \otimes i d_{C}\right)\left(i d_{M} \otimes \mu\right) \\
& +(-1)^{|f|} g\left(i d_{N} \otimes \delta\right)\left(f \otimes i d_{C}\right)\left(i d_{M} \otimes \mu\right) \\
= & g * f\left(i d_{M} \otimes \delta\right)+(-1)^{|f|} g\left(i d_{N} \otimes \delta\right) * f \\
= & g * \underline{\hat{\delta}}(f)+(-1)^{|f|} \underline{\hat{\delta}}(g) * f .
\end{aligned}
$$

Then,

$$
\begin{aligned}
\hat{\delta}(g * f) & =(-1)^{|f|+|g|+1} \hat{\hat{\delta}}(g * f) \\
& =(-1)^{|f|+|g|+1}\left[g * \underline{\hat{\delta}}(f)+(-1)^{|f|} \underline{\hat{\delta}}(g) * f\right] \\
& =\hat{\delta}(g) * f+(-1)^{|g|} g * \hat{\delta}(f) .
\end{aligned}
$$

Definition 2.5. Let $\mathcal{B}=(C, \mu, \epsilon, \delta)$ be a differential graded $S$-bocs. Then, a twisted $\mathcal{B}$-module is a pair $(M, u)$, where $M \in$ GMod- $\mathcal{B}$ and $u$ is a homogeneous morphism $u \in \operatorname{Hom}_{\text {GMod- } \mathcal{B}}^{1}(M, M)$ such that the following Maurer-Cartan equation for $u$ holds

$$
\hat{\delta}(u)+u * u=0 .
$$

If $(M, u)$ and $(N, v)$ are twisted $\mathcal{B}$-modules, then a homogeneous morphism of twisted $\mathcal{B}$-modules $f:(M, u) \longrightarrow(N, v)$ of degree $d$ is a homogeneous morphism $f: M \longrightarrow N$ in GMod- $\mathcal{B}$ of degree $d$ such that

$$
\hat{\delta}(f)+v * f-(-1)^{d} f * u=0 .
$$


We will denote by $\operatorname{Hom}_{\text {GMod- } \mathcal{B}}^{d}((M, u),(N, v))$ the space of homogeneous morphisms of twisted $\mathcal{B}$-modules of degree $d$. Moreover, we make

$$
\operatorname{Hom}_{\text {TGMod- } \mathcal{B}}((M, u),(N, v)):=\bigoplus_{d \in \mathbb{Z}} \operatorname{Hom}_{\text {TGMod- } \mathcal{B}}^{d}((M, u),(N, v)) .
$$

Lemma 2.6. With the preceding notations, we can form the graded category of twisted $\mathcal{B}$-modules TGMod- $\mathcal{B}$ with objects the twisted $\mathcal{B}$-modules, morphisms of twisted $\mathcal{B}$-modules, and the same composition $*$ of the category GMod- $\mathcal{B}$.

Proof. Let $f:(M, u) \longrightarrow(N, v)$ and $g:(N, v) \longrightarrow(L, w)$ be homogeneous morphisms of twisted $\mathcal{B}$-modules. We have $\hat{\delta}(f)+v * f-(-1)^{|f|} f * u=0$ and $\hat{\delta}(g)+w * g-(-1)^{|g|} g * v=0$. Then, we have

$$
\begin{gathered}
\hat{\delta}(g * f)+w * g * f-(-1)^{|g|+|f|} g * f * u= \\
\hat{\delta}(g) * f+(-1)^{|g|} g * \hat{\delta}(f)+w * g * f-(-1)^{|g|+|f|} g * f * u= \\
\hat{\delta}(g) * f+(-1)^{|g|} g *\left[(-1)^{|f|} f * u-v * f\right]+w * g * f-(-1)^{|g|+|f|} g * f * u= \\
\hat{\delta}(g) * f+w * g * f-(-1)^{|g|} g * v * f= \\
{\left[\hat{\delta}(g)+w * g-(-1)^{|g|} g * v\right] * f=0 .}
\end{gathered}
$$

Proposition 2.7. Let $\mathcal{B}_{1}=\left(C_{1}, \mu_{1}, \epsilon_{1}, \delta_{1}\right)$ and $\mathcal{B}_{2}=\left(C_{2}, \mu_{2}, \epsilon_{2}, \delta_{2}\right)$ be differential graded $S$-bocses and $\psi: \mathcal{B}_{1} \longrightarrow \mathcal{B}_{2}$ a morphism of differential graded $S$-bocses. Then, there is a functor of differential graded categories (called the restriction functor associated to the morphism $\psi$ )

$$
R_{\psi}: \text { GMod- } \mathcal{B}_{2} \longrightarrow \text { GMod- } \mathcal{B}_{1}
$$

such that $R_{\psi}(M)=M$, for $M \in \mathrm{GMod}-\mathcal{B}_{2}$, and $R_{\psi}(f)=f\left(i d_{M} \otimes \psi\right)$, for any morphism $f: M \longrightarrow N$ of GMod- $\mathcal{B}_{2}$.

Proof. The identity morphism of $M \in$ GMod- $\mathcal{B}_{2}$ is $\rho\left(i d_{M} \otimes \epsilon_{2}\right): M \otimes_{S} C_{2} \longrightarrow M$, where $\rho: M \otimes_{S} S \longrightarrow M$ is the multiplication map. Then,

$$
R_{\psi}\left(\rho\left(i d_{M} \otimes \epsilon_{2}\right)\right)=\rho\left(i d_{M} \otimes \epsilon_{2}\right)\left(i d_{M} \otimes \psi\right)=\rho\left(i d_{M} \otimes \epsilon_{2} \psi\right)=\rho\left(i d_{M} \otimes \epsilon_{1}\right) .
$$

So, $R_{\psi}$ preserves identities.

Given composable morphisms $f: M \longrightarrow N$ and $g: N \longrightarrow L$ in GMod- $\mathcal{B}_{2}$, we have

$$
\begin{aligned}
R_{\psi}(g * f) & =(g * f)\left(i d_{M} \otimes \psi\right) \\
& =g\left(f \otimes i d_{C_{2}}\right)\left(i d_{M} \otimes \mu_{2}\right)\left(i d_{M} \otimes \psi\right) \\
& =g\left(f \otimes i d_{C_{2}}\right)\left(i d_{M} \otimes \mu_{2} \psi\right) \\
& =g\left(f \otimes i d_{C_{2}}\right)\left(i d_{M} \otimes(\psi \otimes \psi) \mu_{1}\right) \\
& =g\left(f \otimes i d_{C_{2}}\right)\left(i d_{M} \otimes(\psi \otimes \psi)\left(i d_{M} \otimes \mu_{1}\right)\right. \\
& =g\left(i d_{N} \otimes \psi\right)\left(f\left(i d_{M} \otimes \psi\right) \otimes i d_{C_{1}}\right)\left(i d_{M} \otimes \mu_{1}\right) \\
& =g\left(i d_{N} \otimes \psi\right) * f\left(i d_{M} \otimes \psi\right) \\
& =R_{\psi}(g) * R_{\psi}(f) .
\end{aligned}
$$


Finally, given a homogeneous morphism $f: M \longrightarrow N$ in GMod- $\mathcal{B}_{2}$, we have

$$
\begin{aligned}
R_{\psi}\left(\hat{\delta}_{2}(f)\right) & =(-1)^{|f|+1} f\left(i d_{M} \otimes \delta_{2}\right)\left(i d_{M} \otimes \psi\right) \\
& =(-1)^{|f|+1} f\left(i d_{M} \otimes \delta_{2} \psi\right) \\
& =(-1)^{|f|+1} f\left(i d_{M} \otimes \psi \delta_{1}\right) \\
& =(-1)^{\left|R_{\psi}(f)\right|+1} f\left(i d_{M} \otimes \psi\right)\left(i d_{M} \otimes \delta_{1}\right)=\hat{\delta}_{1}\left(R_{\psi}(f)\right) .
\end{aligned}
$$

Proposition 2.8. Let $\mathcal{B}_{1}=\left(C_{1}, \mu_{1}, \epsilon_{1}, \delta_{1}\right)$ and $\mathcal{B}_{2}=\left(C_{2}, \mu_{2}, \epsilon_{2}, \delta_{2}\right)$ be differential graded $S$-bocses and $\psi: \mathcal{B}_{1} \longrightarrow \mathcal{B}_{2}$ a morphism of differential graded $S$-bocses. Then, the restriction functor $R_{\psi}:$ GMod- $\mathcal{B}_{2} \longrightarrow$ GMod- $\mathcal{B}_{1}$ induces a $k$-functor (called again the restriction functor associated to the morphism $\psi$ )

$$
R_{\psi}: \text { TGMod- } \mathcal{B}_{2} \longrightarrow \text { TGMod- } \mathcal{B}_{1}
$$

such that $R_{\psi}(M, u)=\left(M, R_{\psi}(u)\right)$, for $(M, u) \in$ TGMod- $\mathcal{B}_{2}$, and $R_{\psi}(f)=$ $f\left(i d_{M} \otimes \psi\right)$, for any morphism $f:(M, u) \longrightarrow(N, v)$ of TGMod- $\mathcal{B}_{2}$.

Proof. Given $(M, u) \in$ TGMod- $\mathcal{B}_{2}$, we have

$$
\hat{\delta}_{1}\left(R_{\psi}(u)\right)+R_{\psi}(u) * R_{\psi}(u)=R_{\psi}\left(\hat{\delta}_{2}(u)+u * u\right)=0,
$$

so, $\left(M, R_{\psi}(u)\right) \in$ TGMod- $\mathcal{B}_{1}$.

Given a homogeneous morphism $f:(M, u) \longrightarrow(N, v)$ in TGMod- $\mathcal{B}_{2}$, we have that

$$
\hat{\delta}_{1}\left(R_{\psi}(f)\right)+R_{\psi}(v) * R_{\psi}(f)-(-1)^{\left|R_{\psi}(f)\right|} R_{\psi}(f) * R_{\psi}(u)
$$

coincides with

$$
R_{\psi}\left(\hat{\delta}_{2}(f)+v * f-(-1)^{|f|} f * u\right)=0 .
$$

So $R_{\psi}(f):\left(M, R_{\psi}(u)\right) \longrightarrow\left(N, R_{\psi}(v)\right)$ is a homogeneous morphism of twisted $\mathcal{B}_{1}$-modules.

\section{Bocses, coalgebras and homotopy}

Let us see how that the preceding notions relate to the category of comodules and differential comodules over a given differential graded $S$-bocs $\mathcal{B}$. We first recall some basic notions.

Definition 3.1. Given a graded $S$-coalgebra $\mathcal{B}=(C, \mu, \epsilon)$, that is a graded $S$ bocs, we will denote by GComod- $\mathcal{B}$ the category of the graded right $\mathcal{B}$-comodules. Recall that a graded right $\mathcal{B}$-comodule is a pair $\left(M, \mu_{M}\right)$, where $M$ is a graded right $S$-module and $\mu_{M}: M \longrightarrow M \otimes_{S} C$ is a homogeneous morphism of right $S$-modules of degree 0 such that the following diagrams commute

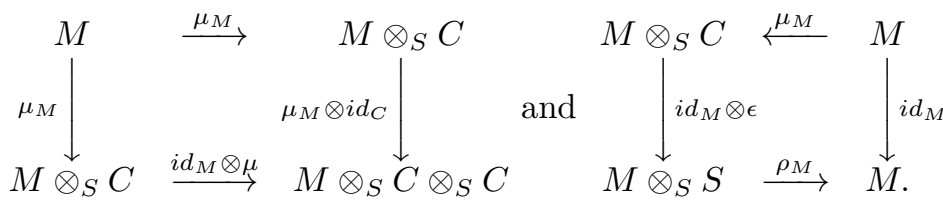


If $\left(M, \mu_{M}\right)$ and $\left(N, \mu_{N}\right)$ are graded right $\mathcal{B}$-comodules and $d \in \mathbb{Z}$, a morphism of graded right $\mathcal{B}$-comodules $h:\left(M, \mu_{M}\right) \longrightarrow\left(N, \mu_{N}\right)$ of degree $d$ is a homogeneous morphism of right $S$-modules $h: M \longrightarrow N$ with degree $d$, such that the following square commutes

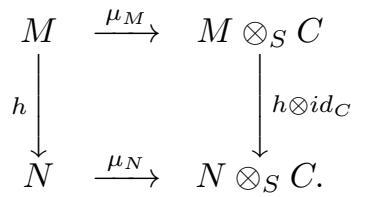

We denote by $\operatorname{Hom}_{\text {GComod- }}^{d}(M, N)$ the space of morphisms of graded right $\mathcal{B}$-comodules of degree $d$ from $M$ to $N$ and we make

$$
\operatorname{Hom}_{\mathrm{GComod}-\mathcal{B}}(M, N):=\bigoplus_{d \in \mathbb{Z}} \operatorname{Hom}_{\mathrm{GComod}-\mathcal{B}}^{d}(M, N) .
$$

We denote by GComod ${ }^{0}-\mathcal{B}$ the subcategory of GComod- $\mathcal{B}$ with the same objects and only degree zero morphisms.

Definition 3.2. Given a differential graded $S$-coalgebra $\mathcal{B}=(C, \mu, \epsilon, \delta)$, that is a differential graded $S$-bocs, we will denote by DGComod- $\mathcal{B}$ the graded category of the differential graded right $\mathcal{B}$-comodules. Recall that a differential graded right $\mathcal{B}$-comodule is a triple $\left(M, \mu_{M}, \delta_{M}\right)$, where $\left(M, \mu_{M}\right)$ is a graded right $\mathcal{B}$ comodule and $\delta_{M}: M \longrightarrow M$ is a differential on $\left(M, \mu_{M}\right)$. Recall that a map $\delta_{M}: M \longrightarrow M$ is a coderivation on $\left(M, \mu_{M}\right)$ iff it is a homogeneous morphism of graded right $S$-modules of degree 1 such that the following diagram commutes

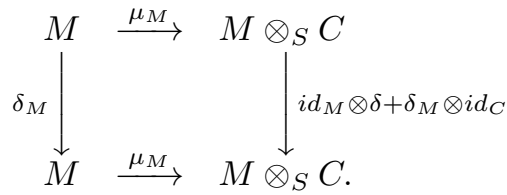

Such a map $\delta_{M}$ is called a differential if, furthermore, $\delta_{M}^{2}=0$.

Whenever $\left(M, \mu_{M}, \delta_{M}\right)$ and $\left(N, \mu_{N}, \delta_{N}\right)$ are differential graded right $\mathcal{B}$-comodules and $d \in \mathbb{Z}$, we agree that a morphism of differential graded right $\mathcal{B}$ comodules $h:\left(M, \mu_{M}, \delta_{M}\right) \longrightarrow\left(N, \mu_{N}, \delta_{N}\right)$ of degree $d$ is a homogeneous morphism of graded right $\mathcal{B}$-comodules $h: M \longrightarrow N$ with degree $d$, such that $\delta_{N} h=(-1)^{d} h \delta_{M}$. We denote by $\operatorname{Hom}_{\text {DGComod- } \mathcal{B}}^{d}(M, N)$ the space of homogeneous morphisms of differential graded right $\mathcal{B}$-comodules of degree $d$ from $M$ to $N$ and we make

$$
\operatorname{Hom}_{\text {DGComod- } \mathcal{B}}(M, N):=\bigoplus_{d \in \mathbb{Z}} \operatorname{Hom}_{\text {DGComod- } \mathcal{B}}^{d}(M, N) .
$$

As before, with DGComod ${ }^{0}-\mathcal{B}$ we denote the subcategory of DGComod- $\mathcal{B}$ with the same objects but only degree zero morphisms. 
Remark 3.3. Let $\mathcal{B}=(C, \mu, \epsilon, \delta)$ be a differential graded $S$-coalgebra, $M, N \in$ DGComod- $\mathcal{B}$, and $h: M \longrightarrow N$ a homogeneous morphism of graded right $\mathcal{B}$ comodules. Then,

$$
\delta_{N} h-(-1)^{|h|} h \delta_{M} \in \operatorname{Hom}_{\text {DGComod-B }}^{|h|+1}(M, N) .
$$

Proposition 3.4. Let $\mathcal{B}=(C, \mu, \epsilon, \delta)$ be a differential graded $S$-bocs.

1. There is a full and faithful functor of graded categories

$$
\Phi: \text { GMod- } \mathcal{B} \longrightarrow \text { GComod- } \mathcal{B}
$$

such that $\Phi(M)=\operatorname{Ind}_{\mathcal{B}}(M):=\left(M \otimes_{S} C, i d_{M} \otimes \mu\right)$, for $M \in$ GMod- $\mathcal{B}$, and, for any $f \in \operatorname{Hom}_{\mathrm{GMod}-\mathcal{B}}(M, N)$, we have $\Phi(f)=\left(f \otimes i d_{C}\right)\left(i d_{M} \otimes \mu\right)$.

2. There is a full and faithful functor of graded categories

$$
\Phi: \text { TGMod- } \mathcal{B} \longrightarrow \text { DGComod- } \mathcal{B}
$$

such that $\Phi(M, u)=\left(\operatorname{Ind}_{\mathcal{B}}(M), \delta_{\Phi(M, u)}\right)$, where $\delta_{\Phi(M, u)}=i d_{M} \otimes \delta+\Phi(u)$, for each $(M, u) \in$ TGMod- $\mathcal{B}$, and $\Phi(f)=\left(f \otimes i d_{C}\right)\left(i d_{M} \otimes \mu\right)$, for any $f \in \operatorname{Hom}_{\text {TGMod-B }}((M, u),(N, v))$.

Proof. (1): It is easy to see that the association $\Phi$ is well defined. In order to show that $\Phi$ preserves composition, take $f \in \operatorname{Hom}_{\mathrm{GMod}-\mathcal{B}}(M, N)$ and $g \in$ $\operatorname{Hom}_{\mathrm{GMod}-\mathcal{B}}(N, L)$. Then,

$$
\begin{aligned}
\Phi(g) \Phi(f) & =\left(g \otimes i d_{C}\right)\left(i d_{N} \otimes \mu\right)\left(f \otimes i d_{C}\right)\left(i d_{M} \otimes \mu\right) \\
& =\left(g \otimes i d_{C}\right)(f \otimes \mu)\left(i d_{M} \otimes \mu\right) \\
& =\left(g \otimes i d_{C}\right)\left(f \otimes i d_{C} \otimes i d_{C}\right)\left(i d_{M} \otimes i d_{C} \otimes \mu\right)\left(i d_{M} \otimes \mu\right) \\
& =\left(g \otimes i d_{C}\right)\left(f \otimes i d_{C} \otimes i d_{C}\right)\left(i d_{M} \otimes\left(i d_{C} \otimes \mu\right) \mu\right) \\
& =\left(g \otimes i d_{C}\right)\left(f \otimes i d_{C} \otimes i d_{C}\right)\left(i d_{M} \otimes\left(\mu \otimes i d_{C}\right) \mu\right) \\
& =\left(g \otimes i d_{C}\right)\left(f \otimes i d_{C} \otimes i d_{C}\right)\left(i d_{M} \otimes \mu \otimes i d_{C}\right)\left(i d_{M} \otimes \mu\right) \\
& =\left(g\left(f \otimes i d_{C}\right)\left(i d_{M} \otimes \mu\right) \otimes i d_{C}\right)\left(i d_{M} \otimes \mu\right) \\
& =\left(g * f \otimes i d_{C}\right)\left(i d_{M} \otimes \mu\right)=\Phi(g * f) .
\end{aligned}
$$

Given $M \in$ GMod- $\mathcal{B}$, we consider the identity morphism $\mathbb{I}_{M}=\rho_{M}\left(i d_{M} \otimes \epsilon\right)$ in the category GMod- $\mathcal{B}$. Then,

$$
\begin{aligned}
\Phi\left(\mathbb{I}_{M}\right) & =\left(\rho_{M}\left(i d_{M} \otimes \epsilon\right) \otimes i d_{C}\right)\left(i d_{M} \otimes \mu\right) \\
& =\left(i d_{M} \otimes \lambda\left(\epsilon \otimes i d_{C}\right)\right)\left(i d_{M} \otimes \mu\right) \\
& =i d_{M} \otimes \lambda\left(\epsilon \otimes i d_{C}\right) \mu=i d_{M} \otimes i d_{C} \\
& =i d_{\operatorname{Ind}_{\mathcal{B}}(M)}=i d_{\Phi(M)} .
\end{aligned}
$$

Thus $\Phi$ is a degree preserving functor. It is full and faithful because, for $d \in \mathbb{Z}$,

$$
\Phi_{d}: \operatorname{Hom}_{\text {GMod-S }}^{d}\left(M \otimes_{S} C, N\right) \longrightarrow \operatorname{Hom}_{\text {GComod- } \mathcal{B}}^{d}\left(\operatorname{Ind}_{\mathcal{B}}(M), \operatorname{Ind}_{\mathcal{B}}(N)\right)
$$

is an isomorphism, with inverse $\Phi_{d}^{\prime}$ given by $\Phi_{d}^{\prime}(\phi)=q_{N} \phi$, where $q_{N}:=$ $\rho_{N}\left(i d_{N} \otimes \epsilon\right)$. 
(2): Take $(M, u) \in$ TGMod- $\mathcal{B}$. If we denote by $\operatorname{Coder}\left(\operatorname{Ind}_{\mathcal{B}}(M)\right)$ the set of coderivations of $\operatorname{Ind}_{\mathcal{B}}(M)$, we have a bijection

$$
\Theta: \operatorname{Hom}_{\text {GComod- } \mathcal{B}}^{1}\left(\operatorname{Ind}_{\mathcal{B}}(M), \operatorname{Ind}_{\mathcal{B}}(M)\right) \longrightarrow \operatorname{Coder}\left(\operatorname{Ind}_{\mathcal{B}}(M)\right)
$$

given by $\Theta(h)=i d_{M} \otimes \delta+h$. Then, we know that $\delta_{\Phi(M, u)}=\Theta \Phi_{1}(u)$ is a coderivation on $\operatorname{Ind}_{\mathcal{B}}(M)$. Since $\delta_{\Phi(M, u)}^{2}$ is a homogeneous morphism of graded right $\mathcal{B}$-comodules of degree 2 , to show that $\delta_{\Phi(M, u)}^{2}=0$ is equivalent to show that $q_{M} \delta_{\Phi(M, u)}^{2}=0$.

From (2.2), we have

$$
\begin{aligned}
q_{M} \delta_{\Phi(M, u)}^{2} & =q_{M}\left[\left(i d_{M} \otimes \delta\right) \Phi(u)+\Phi(u)\left(i d_{M} \otimes \delta\right)+\Phi(u) \Phi(u)\right] \\
& =u\left(i d_{M} \otimes \delta\right)+u \Phi(u)=\hat{\delta}(u)+u * u=0
\end{aligned}
$$

Now, take a homogeneous morphism $f:(M, u) \longrightarrow(N, v)$ in TGMod- $\mathcal{B}$. We already know that $\Phi(f): \operatorname{Ind}_{\mathcal{B}}(M) \longrightarrow \operatorname{Ind}_{\mathcal{B}}(N)$ is a homogeneous morphism of graded $\mathcal{B}$-comodules. By definition, the map $\Phi(f): \Phi(M, u) \longrightarrow \Phi(N, v)$ is a morphism of differential graded $\mathcal{B}$-comodules if and only if the following difference $D$ is zero.

$$
D:=\left(i d_{N} \otimes \delta+\Phi(v)\right) \Phi(f)-(-1)^{|f|} \Phi(f)\left(i d_{M} \otimes \delta+\Phi(u)\right) .
$$

By (3.3), we have that $D=0$ is equivalent to $q_{N} D=0$. Since $f$ is a morphism of twisted $\mathcal{B}$-modules, we have

$$
\begin{aligned}
q_{N} D & =v \Phi(f)-(-1)^{|f|} f\left(i d_{M} \otimes \delta\right)-(-1)^{|f|} f \Phi(u) \\
& =\hat{\delta}(f)+v \Phi(f)-(-1)^{|f|} f \Phi(u) \\
& =\hat{\delta}(f)+v * f-(-1)^{|f|} f * u=0 .
\end{aligned}
$$

Hence $\Phi$ is a well defined faithful functor. In order to show that it is a full functor, we use again the fact that $\Phi_{d}$ is a bijection and reverse the preceding argument.

Definition 3.5. Let $\mathcal{B}=(C, \mu, \epsilon, \delta)$ be a differential graded $S$-bocs. Given morphisms $f, g \in \operatorname{Hom}_{\text {TGMod- } \mathcal{B}}^{0}((M, u),(N, v))$, a homotopy $h$ from $f$ to $g$ is a morphism $h \in \operatorname{Hom}_{\text {GMod- }}^{-1}(M, N)$ such that

$$
f-g=\hat{\delta}(h)+v * h+h * u .
$$

A morphism $f \in \operatorname{Hom}_{\text {TGMod-B }}^{0}((M, u),(N, v))$ is null-homotopic iff there is a homotopy from $f$ to 0 .

We denote by TGMod $^{0}-\mathcal{B}$ the subcategory TGMod- $\mathcal{B}$ with the same objects and only zero degree homogeneous morphisms. Thus, the notion of homotopy is an equivalence relation in the category $\mathrm{TGMod}^{0}-\mathcal{B}$.

Proposition 3.6. Let $\mathcal{B}=(C, \mu, \epsilon, \delta)$ be a differential graded $S$-bocs. Consider the full and faithful functor $\Phi:$ TGMod- $\mathcal{B} \longrightarrow$ DGComod- $\mathcal{B}$ of (3.4). Then, a morphism $f \in \operatorname{Hom}_{\text {TGMod-B }}^{0}((M, u),(N, v))$ in $\operatorname{TGMod}^{0}-\mathcal{B}$ is is null-homotopic 
iff $\Phi(f): \Phi(M, u) \longrightarrow \Phi(N, v)$ is null-homotopic in DGComod ${ }^{0}-\mathcal{B}$. As a consequence, there is an induced full and faithful functor on the homotopy categories

$$
\underline{\Phi}:{\underline{\mathrm{TGMod}^{0}}}^{0}-\mathcal{B} \longrightarrow \underline{\mathrm{DGComod}}^{0}-\mathcal{B} .
$$

Proof. Recall that, by definition, $\Phi(M, u)=\left(\operatorname{Ind}_{\mathcal{B}}(M), i d_{M} \otimes \delta+\Phi(u)\right)$ and $\Phi(N, v)=\left(\operatorname{Ind}_{\mathcal{B}}(N), i d_{N} \otimes \delta+\Phi(v)\right)$. The morphism $\Phi(f)$ is null-homotopic in $\operatorname{DGComod}^{0}-\mathcal{B}$ iff there is a morphism $\Phi(h) \in \operatorname{Hom}_{\mathrm{GComod}-\mathcal{B}}^{-1}\left(\operatorname{Ind}_{\mathcal{B}}(M), \operatorname{Ind}_{\mathcal{B}}(N)\right)$ with

$$
\Phi(f)=\left(i d_{N} \otimes \delta+\Phi(v)\right) \Phi(h)+\Phi(h)\left(i d_{M} \otimes \delta+\Phi(u)\right) .
$$

This is equivalent to

$$
q_{N} \Phi(f)=q_{N}\left[\left(i d_{N} \otimes \delta+\Phi(v)\right) \Phi(h)+\Phi(h)\left(i d_{M} \otimes \delta+\Phi(u)\right)\right],
$$

and this is equivalent to

$$
f=v \Phi(h)+h\left(i d_{M} \otimes \delta\right)+h \Phi(u)=\hat{\delta}(h)+v * h+h * u,
$$

that is, $f$ is null-homotopic in $\operatorname{TGMod}^{0}-\mathcal{B}$.

Lemma 3.7. Let $\mathcal{B}_{1}=\left(C_{1}, \mu_{1}, \epsilon_{1}, \delta_{1}\right)$ and $\mathcal{B}_{2}=\left(C_{2}, \mu_{2}, \epsilon_{2}, \delta_{2}\right)$ be differential graded $S$-bocses and $\psi: \mathcal{B}_{1} \longrightarrow \mathcal{B}_{2}$ a morphism of differential graded $S$ bocses. Then, the restriction functor $R_{\psi}: \mathrm{TGMod}^{0}-\mathcal{B}_{2} \longrightarrow \mathrm{TGMod}^{0}-\mathcal{B}_{1}$ maps null-homotopic morphisms onto null-homotopic morphisms. Hence it induces a $k$-functor on the corresponding homotopy categories

$$
\underline{R}_{\psi}:{\underline{\mathrm{TGMod}^{0}}}^{0} \mathcal{B}_{2} \longrightarrow \underline{\mathrm{TGMod}}^{0}-\mathcal{B}_{1}
$$

Proof. Given a null-homotopic morphism $f \in \operatorname{Hom}_{\text {TGMod- } \mathcal{B}_{2}}^{0}((M, u),(N, v))$, there is $h \in \operatorname{Hom}_{\mathrm{GMod}-\mathcal{B}_{2}}^{-1}(M, N)$ with $f=\hat{\delta}_{2}(h)+v * h+h * u$. Then, applying $R_{\psi}$, we obtain

$$
\begin{aligned}
R_{\psi}(f) & =R_{\psi}\left(\hat{\delta}_{2}(h)\right)+R_{\psi}(v * h)+R_{\psi}(h * u) \\
& =\hat{\delta}_{1}\left(R_{\psi}(h)\right)+R_{\psi}(v) * R_{\psi}(h)+R_{\psi}(h) * R_{\psi}(u) .
\end{aligned}
$$

Hence, $R_{\psi}(f)$ is null-homotopic.

\section{Triangular bocses}

In this section we show some properties of the category TGMod- $\mathcal{B}$, where $\mathcal{B}$ is a differential graded $S$-bocs of a special type, which we call triangular. We stress the fact that some proofs are inspired in the study of differential graded tensor algebras (or ditalgebras) and their module categories initiated by the Kiev school of representation theory, see [2].

Definition 4.1. A normal graded $S$-bocs $\mathcal{B}$ is a differential unitary graded $S$ coalgebra $(C, \mu, \epsilon, \delta)$, with an $S$-S-bimodule decomposition $C=S \bigoplus \bar{C}$ such that $\epsilon: C \longrightarrow S$ is the projection map and $\mu(1)=1 \otimes 1$. 
Lemma 4.2. If $\mathcal{B}=(C, \mu, \epsilon, \delta)$ is a normal graded $S$-bocs, then we have a well defined map

$$
\bar{\mu}: \bar{C} \longrightarrow \bar{C} \otimes_{S} \bar{C} \text { with } \bar{\mu}(x)=\mu(x)-1 \otimes x-x \otimes 1,
$$

and the differential $\delta$ restricts to a map $\bar{\delta}: \bar{C} \longrightarrow \bar{C}$ of degree 1 . Moreover, the triple $\overline{\mathcal{B}}=(\bar{C}, \bar{\mu}, \bar{\delta})$ is a differential graded $S$-coalgebra (without counit). We call $\overline{\mathcal{B}}$ the reduced differential graded $S$-bocs of $\mathcal{B}$.

Proof. See, for instance, [2] (3.2).

The following reinterpretation of the $k$-category GMod- $\mathcal{B}$ is useful.

Definition 4.3. Given a normal graded $S$-bocs $\mathcal{B}=(C, \mu, \epsilon, \delta)$, we can form the following graded category GMod- $\mathcal{B}$. Its objects are the graded right $S$ modules and, given $d \in \mathbb{Z}$, a morphism $f: M \longrightarrow N$ of degree $d$ in GMod- $\mathcal{B}$ is a pair of maps $f=\left(f^{0}, f^{1}\right)$, where $f^{0}: M \longrightarrow N$ and $f^{1}: M \otimes_{S} \bar{C} \longrightarrow N$ are homogeneous morphisms of right $S$-modules of degree $d$. Thus, its hom spaces are given by

$$
\operatorname{Hom}_{\text {GMod- } \mathcal{B}}(M, N)=\operatorname{Hom}_{\text {GMod- } S}(M, N) \times \operatorname{Hom}_{\text {GMod-S }}\left(M \otimes_{S} \bar{C}, N\right) .
$$

If $f=\left(f^{0}, f^{1}\right) \in \operatorname{Hom}_{\text {GMod-B }}(M, N)$ and $g=\left(g^{0}, g^{1}\right) \in \operatorname{Hom}_{\text {GMod-B }}(N, L)$, their composition $g * f=\left((g * f)^{0},(g * f)^{1}\right) \in \operatorname{Hom}_{\mathrm{GM} M-\mathcal{B}}(M, L)$ is defined by $(g * f)^{0}=g^{0} f^{0}$ and $(g * f)^{1}$ is the following composition in GMod- $S$

$$
(g * f)^{1}=g^{1}\left(f^{0} \otimes i d_{\bar{C}}\right)+g^{0} f^{1}+g^{1}\left[\left(f^{1} \otimes i d_{\bar{C}}\right)\left(i d_{M} \otimes \bar{\mu}\right)\right] .
$$

It is not hard to show that GMod- $\mathcal{B}$ is indeed a graded $k$-category, where the identity morphism $\mathbb{I}_{M}$ on each $M \in \operatorname{GMod}-\mathcal{B}$ is just $\left(i d_{M}, 0\right)$. Again, with $\operatorname{GMod}^{0}-\mathcal{B}$, we denote the subcategory of GMod- $\mathcal{B}$ with the same objects but with only zero degree morphisms.

Lemma 4.4. For any normal graded $S$-bocs $\mathcal{B}$, there is an equivalence of graded categories

$$
F: \text { GMod- } \mathcal{B} \longrightarrow \text { GMod- } \mathcal{B}
$$

such that $F(M)=M$, for each $M \in$ GMod- $\mathcal{B}$ and, given any morphism $f \in$ $\operatorname{Hom}_{\text {GMod-B }}(M, N)=\operatorname{Hom}_{\text {GMod-S }}\left(M \otimes_{S} C, N\right)$, we have $F(f)=\left(f^{0}, f^{1}\right)$, where $f^{0}: M \longrightarrow N$ is given by $f^{0}(m)=f(m \otimes 1)$, for $m \in M$, and the morphism $f^{1}: M \otimes_{S} \bar{C} \longrightarrow N$ is just the restriction of the map $f$.

Proof. It is clear that $F$ defined as above determines a linear isomorphism

$$
\operatorname{Hom}_{\mathrm{GMod}-\mathcal{B}}(M, N) \longrightarrow \operatorname{Hom}_{\mathrm{GMod}-\mathcal{B}}(M, N) \text {. }
$$

So we only have to show that $F$ preserves compositions and identities. Assume that $f: M \longrightarrow N$ and $g: N \longrightarrow L$ are morphisms in GMod- $\mathcal{B}$. Then, for $m \in M$, we have

$$
\begin{aligned}
F(g * f)^{0}(m) & =g\left(f \otimes i d_{C}\right)\left(i d_{M} \otimes \mu\right)(m \otimes 1) \\
& =g\left(f \otimes i d_{C}\right)(m \otimes 1 \otimes 1) \\
& =g(f(m \otimes 1) \otimes 1)=g^{0} f^{0}(m) .
\end{aligned}
$$


For $x \in \bar{C}$, we have $\mu(x)=\bar{\mu}(x)+1 \otimes x+x \otimes 1$ and $\bar{\mu}(x)=\sum_{j} y_{j} \otimes z_{j}$, so

$$
\begin{aligned}
F(g * f)^{1}(m \otimes x)= & g\left(f \otimes i d_{C}\right)\left(i d_{M} \otimes \mu\right)(m \otimes x) \\
= & g\left(f \otimes i d_{C}\right)(m \otimes \mu(x)) \\
= & g\left(f \otimes i d_{C}\right)(m \otimes \bar{\mu}(x)) \\
& +g\left(f \otimes i d_{C}\right)(m \otimes x \otimes 1)+g\left(f \otimes i d_{C}\right)(m \otimes 1 \otimes x) \\
= & \sum_{j} g\left(f\left(m \otimes y_{j}\right) \otimes z_{j}\right) \\
= & +g(f(m \otimes x) \otimes 1)+g(f(m \otimes 1) \otimes x) \\
= & \sum_{j} g^{1}\left(f^{1}\left(m \otimes y_{j}\right) \otimes z_{j}\right)+g^{0}\left(f^{1}(m \otimes x)\right)+g^{1}\left(f^{0}(m) \otimes x\right) \\
= & {\left[g^{1}\left(f^{1} \otimes i d_{\bar{C}}\right)\left(i d_{M} \otimes \bar{\mu}\right)+g^{0} f^{1}+g^{1}\left(f^{0} \otimes i d_{\bar{C}}\right)\right](m \otimes x) } \\
= & {[F(g) * F(f)]^{1}(m \otimes x) . }
\end{aligned}
$$

Thus, we obtain that $F(g * f)=F(g) * F(f)$. Given $M \in$ GMod- $\mathcal{B}$, recall that $\mathbb{I}_{M}=\rho_{M}\left(i d_{M} \otimes \epsilon\right)$, thus $F\left(\mathbb{I}_{M}\right)^{0}(m)=\rho\left(i d_{M} \otimes \epsilon\right)(m \otimes 1)=m$. For $x \in \bar{C}$, we have $F\left(\mathbb{I}_{M}\right)^{1}(m \otimes x)=\rho\left(i d_{M} \otimes \epsilon\right)(m \otimes x)=0$. Hence, $F\left(\mathbb{I}_{M}\right)=\left(i d_{M}, 0\right)=$ $\mathbb{I}_{F(M)}$.

Remark 4.5. Given $f: M \longrightarrow N$ in GMod- $\mathcal{B}$, we often refer to the maps $f^{0}$ and $f^{1}$ in $F(f)=\left(f^{0}, f^{1}\right)$ as the components of $f$.

Notice that any morphism $\left(f^{0}, f^{1}\right) \in \operatorname{Hom}_{\mathrm{GMod}-\mathcal{B}}(M, N)$ decomposes as a sum of morphisms $\left(f^{0}, f^{1}\right)=\left(f^{0}, 0\right)+\left(0, f^{1}\right)$ in $\operatorname{Hom}_{\mathrm{GMod}-\mathcal{B}}(M, N)$.

This notation as pairs of maps was relevant in the study of categories of modules over differential tensor algebras in [2. We exploit it here in the context of graded $S$-bocses.

Definition 4.6. A normal graded $S$-bocs $\mathcal{B}=(C, \mu, \epsilon, \delta)$ is called triangular iff there is a sequence

$$
0=\bar{C}_{0} \subseteq \bar{C}_{1} \subseteq \cdots \subseteq \bar{C}_{i} \subseteq \bar{C}_{i+1} \subseteq \cdots
$$

of graded $S$-S-subbimodules of $\bar{C}$ such that $\bar{C}=\bigcup_{i \in \mathbb{N}} \bar{C}_{i}$,

$$
\bar{\mu}\left(\bar{C}_{i}\right) \subseteq \bar{C}_{i-1} \otimes_{S} \bar{C}_{i-1} \text { and } \bar{\delta}\left(\bar{C}_{i}\right) \subseteq \bar{C}_{i} \text {, for all } i \in \mathbb{N} .
$$

Proposition 4.7. Let $\mathcal{B}=(C, \mu, \epsilon, \delta)$ be a triangular graded $S$-bocs. Consider any endomorphism in $\mathrm{GMod}^{0}-\mathcal{B}$ of the form $f=\left(0, f^{1}\right): M \longrightarrow M$. Then, for each $x \in \bar{C}$, there is a natural number $n_{x}$ such that $\left(f^{n}\right)^{1}[m \otimes x]=0$, for all $n \geq n_{x}$ and $m \in M$. In this case, we can consider the morphism in $\mathrm{GMod}^{0}-\mathcal{B}$

$$
\sum_{n=1}^{\infty} f^{n}: M \longrightarrow M .
$$

Proof. Adopt the notations of (4.6). We proceed to show, by induction on $i \in \mathbb{N}$, that there is a natural number $n_{i}$ such that for any $x \in \bar{C}_{i}$ and $m \in M$, we have, $\left(f^{n_{i}}\right)^{1}[m \otimes x]=0$.

From the definition of the composition of morphisms is GMod- $\mathcal{B}$, we have that $\left(f^{n+1}\right)^{1}(m \otimes x)=f^{n}\left(f^{1} \otimes i d_{\bar{C}}\right)\left(i d_{M} \otimes \bar{\mu}\right)[m \otimes x]=0$, for $x \in \bar{C}_{1}$ and $n \geq 1$, and we can take $n_{1}=2$. 
Assume that we have proved that $n_{i}$ exists for a fixed $i \in \mathbb{N}$, such that for any $x \in \bar{C}_{i}$ and $m \in M$, we have $\left(f^{n_{i}}\right)^{1}[m \otimes x]=0$. Then, if $x \in \bar{C}_{i+1}$, we have that $\bar{\mu}(x)=\sum_{j=1}^{t} x_{j} \otimes y_{j}$, with $x_{j}, y_{j} \in \bar{C}_{i}$. Thus, for $m \in M$, we have

$$
\begin{aligned}
\left(f^{n_{i}+1}\right)^{1}[m \otimes x] & =f^{n_{i}}\left(f^{1} \otimes i d_{\bar{C}}\right)\left(i d_{M} \otimes \bar{\mu}\right)[m \otimes x] \\
& =f^{n_{i}}\left(f^{1} \otimes i d_{\bar{C}}\right)(m \otimes \bar{\mu}(x)) \\
& =\sum_{j} f^{n_{i}}\left(f^{1} \otimes i d_{\bar{C}}\right)\left(m \otimes x_{j} \otimes y_{j}\right) \\
& =\sum_{j} f^{n_{i}}\left(f^{1}\left(m \otimes x_{j}\right) \otimes y_{j}\right)=0 .
\end{aligned}
$$

The statement of the Proposition follows from this.

Corollary 4.8. Let $\mathcal{B}$ be a triangular graded $S$-bocs. Take any morphism $f=$ $\left(f^{0}, f^{1}\right): M \longrightarrow N$ in $\mathrm{GMod}^{0}-\mathcal{B}$. Then, $f: M \longrightarrow N$ is an isomorphism in $\mathrm{GMod}^{0}-\mathcal{B}$ iff $f^{0}: M \longrightarrow N$ is an isomorphism of GMod-S.

Proof. We first show that an endomorphism of the form $f=\left(i d_{M}, f^{1}\right): M \longrightarrow M$ is an automorphism. Indeed, we can write $f=\mathbb{I}_{M}-h$ where $h=\left(0,-f^{1}\right)$. Then

$$
\left(\mathbb{I}_{M}-h\right) *\left(\sum_{i=0}^{\infty} h^{i}\right)=\sum_{i=0}^{\infty} h^{i}-h *\left(\sum_{i=0}^{\infty} h^{i}\right)=h^{0}=\mathbb{I}_{M} .
$$

Similarly, we have $\left(\sum_{i=0}^{\infty} h^{i}\right) *\left(\mathbb{I}_{M}-h\right)=\mathbb{I}_{M}$. Thus $f$ is an automorphism.

Now, consider any morphism $f=\left(f^{0}, f^{1}\right): M \longrightarrow N$ with $f^{0}: M \longrightarrow N$ isomorphism and consider the inverse $g^{0}: N \longrightarrow M$ of $f^{0}$. Then, $f *\left(g^{0}, 0\right)=$ $\left(i d_{N}, u\right)$ and $\left(g^{0}, 0\right) * f=\left(i d_{M}, v\right)$ are isomorphisms. So, $f$ is a section and a retraction in GMod- $\mathcal{B}$. It follows that $f$ is an isomorphism in GMod- $\mathcal{B}$.

Corollary 4.9. Let $\mathcal{B}$ be a triangular graded $S$-bocs. Take any morphism $f$ : $(M, u) \longrightarrow(N, v)$ in TGMod $^{0}-\mathcal{B}$. Then, $f: M \longrightarrow N$ is an isomorphism in $\operatorname{GMod}^{0}-\mathcal{B}$ iff its first component $f^{0}: M \longrightarrow N$ is an isomorphism of GMod-S.

Proof. From (4.8), it will be enough to show the following.

Claim: Fix any homogeneous morphism $f:(M, u) \longrightarrow(N, v)$ in $\operatorname{TGMod}^{0}-\mathcal{B}$. Then, $f:(M, u) \longrightarrow(N, v)$ is an isomorphism in $\mathrm{TGMod}^{0}-\mathcal{B}$ iff $f: M \longrightarrow N$ is an isomorphism in GMod- $\mathcal{B}$.

We now prove the claim. Since $f:(M, u) \longrightarrow(N, v)$ is a morphism in $\operatorname{TGMod}^{0}-\mathcal{B}$, we have that $\hat{\delta}(f)+v * f-f * u=0$. If $f$ is an isomorphism in $\operatorname{GMod}^{0}-\mathcal{B}$, it has a homogeneous two-sided inverse $g: N \longrightarrow M$ with $|g|=0$. Since $g * f=\mathbb{I}_{M}$, we have $0=\hat{\delta}\left(\mathbb{I}_{M}\right)=\hat{\delta}(g * f)=\hat{\delta}(g) * f+g * \hat{\delta}(f)$. So,

$$
\hat{\delta}(g)=-g * \hat{\delta}(f) * g=g *(v * f) * g-g *(f * u) * g=g * v-u * g .
$$

So, $g:(N, v) \longrightarrow(M, u)$ is a morphism in $\mathrm{TGMod}^{0}-\mathcal{B}$, an inverse for $f$.

Proposition 4.10. Suppose that $\mathcal{B}$ is a triangular differential graded $S$-bocs. Consider a composable pair of morphisms in $\operatorname{GMod}^{0}-\mathcal{B}$

$$
M \stackrel{f}{\longrightarrow} E \stackrel{g}{\longrightarrow} N
$$


such that $g * f=0$ and the sequence $0 \longrightarrow M \stackrel{f^{0}}{\longrightarrow} E \stackrel{g^{0}}{\longrightarrow} N \longrightarrow 0$ is an exact sequence in GMod- $S$. Then, there is a commutative diagram in $\mathrm{GMod}^{0}-\mathcal{B}$

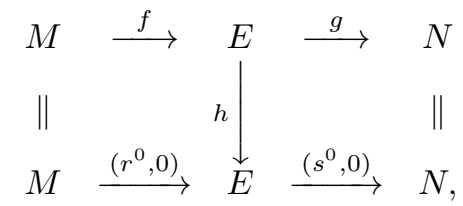

where $h$ is an automorphism.

Proof. Step 1: There is an automorphism $h$ of $E$ such that $(h * f)^{1}=0$.

We have $f=\underline{f}^{0}+\underline{f}^{1}$, where $\underline{f}^{0}=\left(f^{0}, 0\right)$ and $\underline{f}^{1}=\left(0, f^{1}\right)$ are morphisms in $\mathrm{GMod}^{0}-\mathcal{B}$. Since $S$ is semisimple, the exact sequence in the statement of our proposition splits and we have a morphism $p: E \longrightarrow M$ in GMod- $S$ such that $p f^{0}=i d_{M}$. Consider the morphism $\underline{p}=(p, 0): E \longrightarrow M$ in $\operatorname{GMod}^{0}-\mathcal{B}$. Then, we have $\underline{p} * \underline{f}^{0}=\mathbb{I}_{M}$ in GMod- $\mathcal{B}$. Make $u:=f^{1} * \underline{p}$ and notice that $u^{0}=0$. Then, we have the automorphism $h$ of $E$ defined by

$$
h=\mathbb{I}_{E}+v: E \longrightarrow E, \text { where } v=\sum_{n=1}^{\infty}(-1)^{n} u^{n} .
$$

We have that $h * f=\underline{f}^{0}+\underline{f}^{1}+v * \underline{f}^{0}+v * \underline{f}^{1}$, where

$$
\begin{aligned}
\underline{f}^{1}+v * \underline{f}^{0}+v * \underline{f}^{1}= & \underline{f}^{1}-\underline{f}^{1} * \underline{p} * \underline{f}^{0}+\sum_{n=1}^{\infty}(-1)^{n+1}\left(\underline{f}^{1} * \underline{p}\right)^{n} *\left(\underline{f}^{1} * \underline{p}\right) * \underline{f}^{0} \\
& +\sum_{n=1}^{\infty}(-1)^{n}\left(\underline{f}^{1} * \underline{p}\right)^{n} * \underline{f}^{1} \\
= & \underline{f}^{1}-\underline{f}^{1}+\sum_{n=1}^{\infty}(-1)^{n+1}\left(\underline{f}^{1} * \underline{p}\right)^{n} * \underline{f}^{1} \\
& +\sum_{n=1}^{\infty}(-1)^{n}\left(\underline{f}^{1} * \underline{p}^{n} * \underline{f}^{1}=0 .\right.
\end{aligned}
$$

Therefore, we obtain $(h * f)^{1}=0$.

Step 2: Now, we may assume that $f=\left(f^{0}, 0\right)$ and finish the proof.

As before, we have $g=\underline{g}^{0}+\underline{g}^{1}$, where $\underline{g}^{0}=\left(g^{0}, 0\right)$ and $\underline{g}^{1}=\left(0, g^{1}\right)$ are morphisms in $\mathrm{GMod}^{0}-\mathcal{B}$. Since $S$ is semisimple, there is a morphism $s: N \longrightarrow E$ in GMod- $S$ such that $g^{0} s=i d_{N}$. If we take $\underline{s}=(s, 0)$ we have $g^{0} * \underline{s}=\mathbb{I}_{N}$ in GMod- $\mathcal{B}$. Now, make $u=\underline{s} * \underline{g}^{1}$ and notice that $u^{0}=0$. Now, consider the automorphism $h$ of $E$ defined by

$$
h=\mathbb{I}_{E}+w: E \longrightarrow E, \text { where } w=\sum_{i=1}^{\infty}(-1)^{n} u^{n} .
$$


We have that $g * h=\underline{g}^{0}+\underline{g}^{1}+\underline{g}^{0} * w+\underline{g}^{1} * w$, where

$$
\begin{aligned}
\underline{g}^{1}+\underline{g}^{0} * w+\underline{g}^{1} * w= & \underline{g}^{1}-\underline{g}^{0} * \underline{s} * \underline{g}^{1}+\sum_{n=1}^{\infty}(-1)^{n+1} \underline{g}^{0} * \underline{s} * \underline{g}^{1} *\left(\underline{s} * \underline{g}^{1}\right)^{n} \\
& +\sum_{n=1}^{\infty}(-1)^{n} \underline{g}^{1} *\left(\underline{s} * \underline{g}^{1}\right)^{n} \\
= & \underline{g}^{1}-\underline{g}^{1}+\sum_{n=1}^{\infty}(-1)^{n+1} \underline{g}^{1} *\left(\underline{s} * \underline{g}^{1}\right)^{n} \\
& +\sum_{n=1}^{\infty}(-1)^{n} \underline{g}^{1} *\left(\underline{s} * \underline{g}^{1}\right)^{n}=0 .
\end{aligned}
$$

Therefore, we obtain $(g * h)^{1}=0$. Notice that

$$
u+w+w * u=u+\sum_{n=1}^{\infty}(-1)^{n} u^{n}+\sum_{n=1}^{\infty}(-1)^{n} u^{n+1}=0 .
$$

Hence, $h^{-1}=\mathbb{I}_{E}+u$. Since $g * f=0$, we have that $\underline{g}^{1} * f=0$ and, therefore, $h^{-1} * f=f+u * f=f$. So, $\left(h^{-1} * f\right)^{1}=0$ and we are done.

Lemma 4.11. Let $\mathcal{B}$ be a differential graded $S$-bocs, assume that $(M, u) \in$ TGMod- $\mathcal{B}$, and $N \in$ GMod- $\mathcal{B}$. Then, for any homogeneous isomorphism $h$ : $M \longrightarrow N$ in GMod-B $\mathcal{B}$, there is a unique morphism $v \in \operatorname{Hom}_{\text {GMod- } \mathcal{B}}^{1}(N, N)$ such that $h:(M, u) \longrightarrow(N, v)$ is an isomorphism in TGMod- $\mathcal{B}$.

Proof. By assumption, $\hat{\delta}(u)+u * u=0$ and we are looking for a morphism $v \in \operatorname{Hom}_{\text {GMod- } \mathcal{B}}^{1}(N, N)$ such that $\hat{\delta}(h)+v * h-(-1)^{|h|} h * u=0$. Then, the only possible choice is $v=(-1)^{|h|} h * u * h^{-1}-\hat{\delta}(h) * h^{-1}$. It remains to show that $\hat{\delta}(v)+v * v=0$. We shall compute each term separately.

From Leibniz rule, we have that $0=\hat{\delta}\left(\mathbb{I}_{N}\right)=\hat{\delta}\left(h * h^{-1}\right)=\hat{\delta}(h) * h^{-1}+$ $(-1)^{|h|} h * \hat{\delta}\left(h^{-1}\right)$. Then, we have $\hat{\delta}\left(h^{-1}\right)=-(-1)^{|h|} h^{-1} * \hat{\delta}(h) * h^{-1}$, and

$$
\begin{aligned}
\hat{\delta}(v)= & (-1)^{|h|} \hat{\delta}\left(h * u * h^{-1}\right)-\hat{\delta}\left(\hat{\delta}(h) * h^{-1}\right) \\
= & (-1)^{|h|} \hat{\delta}(h) * u * h^{-1}+h * \hat{\delta}(u) * h^{-1} \\
& -h * u * \hat{\delta}\left(h^{-1}\right)+(-1)^{|h|} \hat{\delta}(h) * \hat{\delta}\left(h^{-1}\right) \\
= & (-1)^{|h|} \hat{\delta}(h) * u * h^{-1}+h * \hat{\delta}(u) * h^{-1} \\
& +(-1)^{|h|} h * u * h^{-1} * \hat{\delta}(h) * h^{-1} \\
& -\hat{\delta}(h) * h^{-1} * \hat{\delta}(h) * h^{-1} .
\end{aligned}
$$

Moreover, we have

$$
\begin{aligned}
v * v= & {\left[(-1)^{|h|} h * u * h^{-1}-\hat{\delta}(h) * h^{-1}\right] *\left[(-1)^{|h|} h * u * h^{-1}-\hat{\delta}(h) * h^{-1}\right] } \\
= & h * u * u * h^{-1}-(-1)^{|h|} h * u * h^{-1} * \hat{\delta}(h) * h^{-1} \\
& -(-1)^{|h|} \hat{\delta}(h) * u * h^{-1}+\hat{\delta}(h) * h^{-1} * \hat{\delta}(h) * h^{-1} .
\end{aligned}
$$

Since $\hat{\delta}(u)+u * u=0$, we obtain $\hat{\delta}(v)=-v * v$, as we wanted. 
Proposition 4.12. Suppose that $\mathcal{B}$ is a triangular graded $S$-bocs. Consider a composable pair of homogeneous morphisms in $\mathrm{TGMod}^{0}-\mathcal{B}$

$$
\left(M, u_{M}\right) \stackrel{f}{\longrightarrow}\left(E, u_{E}\right) \stackrel{g}{\longrightarrow}\left(N, u_{N}\right)
$$

such that $g * f=0$ and the sequence of first components

$$
0 \longrightarrow M \stackrel{f^{0}}{\longrightarrow} E \stackrel{g^{0}}{\longrightarrow} N \longrightarrow 0
$$

is exact in GMod-S. Then, there is a commutative diagram in $\mathrm{TGMod}^{0}-\mathcal{B}$

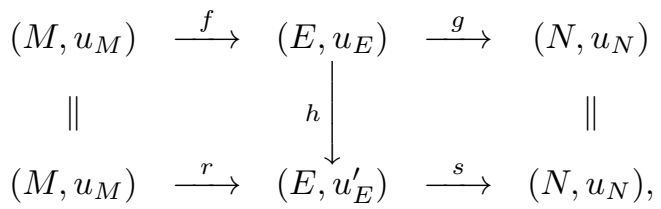

where $h$ is an isomorphism and the second components of $r$ and $s$ are zero.

Proof. Consider the underlying diagrams in $\operatorname{GMod}^{0}-\mathcal{B}$ of our hypothesis. Then, there is an isomorphism $h: E \longrightarrow E$ in $\operatorname{GMod}^{0}-\mathcal{B}$ as in (4.10). We have $\left(E, u_{E}\right) \in$ TGMod- $\mathcal{B}$ and, so, we can apply (4.11) to $h$ and obtain a morphism $u_{E}^{\prime} \in \operatorname{Hom}_{\text {GMod-B }}^{1}(E, E)$ such that $h:\left(E, u_{E}\right) \longrightarrow\left(E, u_{E}^{\prime}\right)$ is an isomorphism in TGMod $^{0}-\mathcal{B}$.

\section{Scalar restriction functors and homotopy}

Let us recall the classical definition of homotopy of differential graded $S$-coalgebras (or $S$-bocses).

Definition 5.1. Let $\overline{\mathcal{B}}_{A}=\left(\bar{C}_{A}, \bar{\mu}_{A}, \bar{\delta}_{A}\right)$ and $\overline{\mathcal{B}}_{B}=\left(\bar{C}_{B}, \bar{\mu}_{B}, \bar{\delta}_{B}\right)$ be differential graded $S$-bocses (without counit). Let $\bar{\phi}, \bar{\psi}: \bar{C}_{A} \longrightarrow \bar{C}_{B}$ be morphisms of differential graded $S$-bocses. A homotopy $\bar{h}$ from $\bar{\phi}$ to $\bar{\psi}$ is a morphism $\bar{h} \in \operatorname{Hom}_{\mathrm{GMod}-S-S}^{-1}\left(\bar{C}_{A}, \bar{C}_{B}\right)$ such that

$$
\bar{\mu}_{B} \bar{h}=(\bar{\phi} \otimes \bar{h}+\bar{h} \otimes \bar{\psi}) \bar{\mu}_{A} \quad \text { and } \quad \bar{\phi}-\bar{\psi}=\bar{\delta}_{B} \bar{h}+\bar{h} \bar{\delta}_{A} .
$$

The morphisms $\bar{\phi}$ and $\bar{\psi}$ are called homotopic iff there is such a homotopy $\bar{h}$. A morphism $\bar{h} \in \operatorname{Hom}_{\text {GMod-S-S }}^{-1}\left(\bar{C}_{A}, \bar{C}_{B}\right)$ is called a $\bar{\phi}-\bar{\psi}$-coderivation when the preceding left equality is satisfied. We denote by $\operatorname{Coder}_{\bar{\phi}-\bar{\psi}}^{-1}\left(\bar{C}_{A}, \bar{C}_{B}\right)$ the space of $\bar{\phi}-\bar{\psi}$-coderivations.

The definition of homotopy of morphisms of differential counitary graded $S$ bocses is similar, we just remove the overlines and add $\epsilon_{A}$ and $\epsilon_{B}$ to the given triples, respectively.

The proof of the following statement is straightforward. 
Lemma 5.2. Let $\mathcal{B}_{A}=\left(C_{A}, \mu_{A}, \epsilon_{A}, \delta_{A}\right)$ and $\mathcal{B}_{B}=\left(C_{B}, \mu_{B}, \epsilon_{B}, \delta_{B}\right)$ be differential normal graded $S$-bocses. Let $\bar{\phi}, \bar{\psi}: \overline{\mathcal{B}}_{A} \longrightarrow \overline{\mathcal{B}}_{B}$ be morphisms of the corresponding reduced differential graded $S$-bocses and assume that $\bar{h}$ is a homotopy from $\bar{\phi}$ to $\bar{\psi}$. Extend $\bar{\phi}$ and $\bar{\psi}$ to morphisms of differential unitary graded $S$ bocses $\phi, \psi: \mathcal{B}_{A} \longrightarrow \mathcal{B}_{B}$ defining $\phi(s+x)=s+\bar{\phi}(x)$ and $\psi(s+x)=s+\bar{\psi}(x)$, for $s \in S$ and $x \in \bar{C}_{A}$; extend $\bar{h}$ to $h: C_{A} \longrightarrow C_{B}$ defining $h(s+x)=\bar{h}(x)$. Then, we have that $h$ is a homotopy from $\phi$ to $\psi$. That is $h \in \operatorname{Hom}_{\mathrm{GMod}-S-S}^{-1}\left(C_{A}, C_{B}\right)$ satisfies

$$
\mu_{B} h=(\phi \otimes h+h \otimes \psi) \mu_{A} \quad \text { and } \quad \phi-\psi=\delta_{B} h+h \delta_{A} .
$$

Lemma 5.3. Under the assumptions of the last lemma, for $d \in \mathbb{Z}$, define

$$
R_{h}: \operatorname{Hom}_{\mathrm{GMod}-\mathcal{B}_{B}}^{d}(M, N) \longrightarrow \operatorname{Hom}_{\mathrm{GMod}-\mathcal{B}_{A}}^{d-1}(M, N)
$$

such that $R_{h}(u)=(-1)^{|u|} u\left(i d_{M} \otimes h\right)$, for $u \in \operatorname{Hom}_{\mathrm{GMod}-\mathcal{B}_{B}}^{d}(M, N)$. Then, the following holds.

1. Whenever $u: M \longrightarrow N$ and $v: N \longrightarrow L$ are homogeneous morphisms in GMod- $\mathcal{B}_{B}$, we have

$$
R_{h}(v * u)=R_{h}(v) * R_{\phi}(u)+(-1)^{|v|} R_{\psi}(v) * R_{h}(u) .
$$

2. For any homogeneous morphism $u: M \longrightarrow N$ in GMod- $\mathcal{B}_{B}$, we have

$$
R_{\phi}(u)-R_{\psi}(u)=\hat{\delta}_{A} R_{h}(u)+R_{h} \hat{\delta}_{B}(u)
$$

Proof. (1): Having in mind (2.7), we get

$$
\begin{aligned}
R_{h}(v * u)= & (-1)^{|u|+|v|} v\left(u \otimes i d_{C_{B}}\right)\left(i d_{M} \otimes \mu_{B}\right)\left(i d_{M} \otimes h\right) \\
= & (-1)^{|u|+|v|} v\left(u \otimes i d_{C_{B}}\right)\left(i d_{M} \otimes \mu_{B} h\right) \\
= & (-1)^{|u|+|v|} v\left(u \otimes i d_{C_{B}}\right)\left(i d_{M} \otimes(\phi \otimes h+h \otimes \psi) \mu_{A}\right) \\
= & (-1)^{|u|+|v|} v\left(u \otimes i d_{C_{B}}\right)\left(i d_{M} \otimes(\phi \otimes h) \mu_{A}\right) \\
= & (-1)^{|u|+|v|} v\left(u \otimes i d_{C_{B}}\right)\left(i d_{M} \otimes(h \otimes \psi) \mu_{A}\right) \\
= & (-1)^{|u|+|v|} v\left(u\left(i d_{M} \otimes \phi\right) \otimes h\right)\left(i d_{M} \otimes \mu_{A}\right) \\
= & (-1)^{|v|+|v|} v\left(u\left(i d_{M} \otimes h\right) \otimes \psi\right)\left(i d_{M} \otimes \mu_{A}\right) \\
& (-1)^{|u|+|v|} v\left(i d_{N} \otimes \psi\right)\left(u\left(i d_{M} \otimes \phi\right) \otimes i d_{C_{A}}\right)\left(i d_{M} \otimes \mu_{A}\right) \\
= & R_{h}(v) * R_{\phi}(u)+(-1)^{|v|} R_{\psi}(v) * R_{h}(u) .
\end{aligned}
$$

(2): Since $\hat{\delta}_{B}(u)=(-1)^{|u|+1} u\left(i d_{M} \otimes \delta_{B}\right)$, we have

$$
\begin{aligned}
R_{h} \hat{\delta}_{B}(u) & =(-1)^{|u|+1}(-1)^{|u|+1} u\left(i d_{M} \otimes \delta_{B}\right)\left(i d_{M} \otimes h\right) \\
& =u\left(i d_{M} \otimes \delta_{B} h\right)=u\left(i d_{M} \otimes\left(\phi-\psi-h \delta_{A}\right)\right) \\
& =u\left(i d_{M} \otimes \phi\right)-u\left(i d_{M} \otimes \psi\right)-u\left(i d_{M} \otimes h\right)\left(i d_{M} \otimes \delta_{A}\right) \\
& =R_{\phi}(u)-R_{\psi}(u)-\hat{\delta}_{A} R_{h}(u) .
\end{aligned}
$$


Proposition 5.4. Adopt the assumptions of (5.2) and assume, furthermore, that $\mathcal{B}_{A}$ is a triangular $S$-bocs. Then, there is an isomorphism of functors

$$
\underline{\eta}: \underline{R}_{\phi} \longrightarrow \underline{R}_{\psi},
$$

where $\underline{R}_{\phi}, \underline{R}_{\psi}: \underline{\text { TGMod }}^{0}-\mathcal{B}_{B} \longrightarrow \underline{\text { TGMod }}^{0}-\mathcal{B}_{A}$ are the functors induced on the homotopy categories by $R_{\phi}, R_{\psi}: \mathrm{TGMod}^{0}-\mathcal{B}_{B} \longrightarrow \mathrm{TGMod}^{0}-\mathcal{B}_{A}$, respectively, see 3.7). For each $(M, u) \in$ TGMod- $\mathcal{B}_{B}$, the map $\underline{\eta}_{(M, u)}: R_{\phi}(M, u) \longrightarrow R_{\psi}(M, u)$ is the class modulo homotopy of the morphism

$$
\eta_{(M, u)}=\mathbb{I}_{M}+R_{h}(u): R_{\phi}(M, u) \longrightarrow R_{\psi}(M, u) .
$$

Proof. We first show that $\mathbb{I}_{M}+R_{h}(u) \in \operatorname{Hom}_{\mathrm{GMod}-\mathcal{B}_{A}}^{0}(M, M)$ is an isomorphism in the category GMod- $\mathcal{B}_{A}$. In order to see this, consider the equivalence $F$ : GMod- $\mathcal{B}_{A} \longrightarrow$ GMod- $\mathcal{B}_{A}$ of (4.4). Consider the morphism $F\left(R_{h}(u)\right)=\left(h^{0}, h^{1}\right)$ : $M \longrightarrow M$ in GMod- $\mathcal{B}$ and notice that, for $m \in M$, we have

$$
h^{0}(m)=R_{h}(u)(m \otimes 1)=-u\left(i d_{M} \otimes h\right)(m \otimes 1)=0,
$$

because $h(1)=0$. Therefore, $h^{0}=0$ and, by (4.8), we know that $\mathbb{I}_{M}+R_{h}(u)$ is an isomorphism in $\mathrm{GMod}^{0}-\mathcal{B}_{A}$.

Now we show that $\mathbb{I}_{M}+R_{h}(u) \in \operatorname{Hom}_{\mathrm{TGMod}-\mathcal{B}_{A}}^{0}\left(R_{\phi}(M, u), R_{\psi}(M, u)\right)$. By definition, this is equivalent to show the identity

$$
(*): \quad \hat{\delta}_{A}\left(\mathbb{I}_{M}+R_{h}(u)\right)+R_{\psi}(u) *\left(\mathbb{I}_{M}+R_{h}(u)\right)-\left(\mathbb{I}_{M}+R_{h}(u)\right) * R_{\phi}(u)=0 .
$$

We know that $u: M \otimes_{S} C_{B} \longrightarrow M$ is a homogeneous morphism of degree $1 \mathrm{such}$ that $\hat{\delta}_{B}(u)+u * u=0$. Then, applying $R_{h}$ and using (5.3), we get

$$
\begin{aligned}
0 & =R_{h}\left(\hat{\delta}_{B}(u)\right)+R_{h}(u * u) \\
& =R_{\phi}(u)-R_{\psi}(u)-\hat{\delta}_{A} R_{h}(u)+R_{h}(u) * R_{\phi}(u)-R_{\psi}(u) * R_{h}(u) .
\end{aligned}
$$

That is $\hat{\delta}_{A} R_{h}(u)+R_{\psi}(u) * R_{h}(u)-R_{h}(u) * R_{\phi}(u)+R_{\psi}(u)-R_{\phi}(u)=0$, which is equivalent to the identity $(*)$.

Finally, we show that the family of classes $\underline{\eta}_{(M, u)}: R_{\phi}(M, u) \longrightarrow R_{\psi}(M, u)$ modulo homotopy of the morphisms $\eta_{(M, u)}=\mathbb{I}_{M}+R_{h}(u): R_{\phi}(M, u) \longrightarrow R_{\psi}(M, u)$ is a natural transformation. Take any homogeneous morphism $f:(M, u) \longrightarrow(N, v)$ of degree 0 in TGMod- $\mathcal{B}_{B}$. Hence, we have $\hat{\delta}_{B}(f)+v * f-f * u=0$. Applying $R_{h}$ to the preceding equality, we obtain

$$
\begin{aligned}
0= & R_{h} \hat{\delta}_{B}(f)+R_{h}(v * f)-R_{h}(f * u) \\
= & R_{\phi}(f)-R_{\psi}(f)-\hat{\delta}_{A} R_{h}(f)+R_{h}(v) * R_{\phi}(f)-R_{\psi}(v) * R_{h}(f) \\
& -R_{h}(f) * R_{\phi}(u)-R_{\psi}(f) * R_{h}(u) .
\end{aligned}
$$

Hence,

$$
\begin{aligned}
\hat{\delta}_{A} R_{h}(f)= & R_{\phi}(f)-R_{\psi}(f)+R_{h}(v) * R_{\phi}(f)-R_{\psi}(v) * R_{h}(f) \\
& -R_{h}(f) * R_{\phi}(u)-R_{\psi}(f) * R_{h}(u) .
\end{aligned}
$$


Let us compute the difference $D=\eta_{(N, v)} * R_{\phi}(f)-R_{\psi}(f) * \eta_{(M, u)}$.

$$
\begin{aligned}
D & =\left(\mathbb{I}_{N}+R_{h}(v)\right) * R_{\phi}(f)-R_{\psi}(f) *\left(\mathbb{I}_{M}+R_{h}(u)\right) \\
& =R_{\phi}(f)-R_{\psi}(f)+R_{h}(v) * R_{\phi}(f)-R_{\psi}(f) * R_{h}(u) \\
& =\hat{\delta}_{A} R_{h}(f)+R_{h}(f) * R_{\phi}(u)+R_{\psi}(v) * R_{h}(f) .
\end{aligned}
$$

Hence, $D$ is null-homotopic in $\operatorname{TGMod}^{0}-\mathcal{B}_{A}$. This finishes the proof.

Corollary 5.5. Let $\phi: \mathcal{B}_{A} \longrightarrow \mathcal{B}_{B}$ be a homotopy equivalence of triangular $S$ bocses. Then, the corresponding restriction functor determines an equivalence of categories

$$
\underline{R}_{\phi}:{\underline{\operatorname{TGMod}^{0}}}^{-} \mathcal{B}_{B} \longrightarrow \underline{\mathrm{TGMod}}^{0}-\mathcal{B}_{A} .
$$

Proof. If $\phi$ is a homotopy equivalence, its class modulo homotopy $\phi$ has an inverse $\psi$, for some morphism of triangular $S$-bocses $\psi: \mathcal{B}_{B} \longrightarrow \mathcal{B}_{A}$. Then we have $\phi \bar{\psi}=\phi \psi \sim \underline{i d}_{\mathcal{B}_{B}}$ and $\psi \phi=\psi \phi \sim \underline{i d}_{\mathcal{B}_{A}}$. Therefore, we get isomorphisms of functors $\underline{\underline{R}}_{\phi} \underline{R}_{\psi}=\underline{R}_{\psi \phi} \cong \overline{i d_{\mathrm{TGMod}^{0}-\mathcal{B}_{A}}}$ and $\underline{R}_{\psi} \underline{R}_{\phi}=\underline{R}_{\phi \psi} \cong i d_{\underline{\mathrm{TGMod}}^{0}-\mathcal{B}_{B}}$.

\section{Splitting idempotents for twisted modules}

In this section we show that idempotents split in the category $\operatorname{TGMod}^{0}-\mathcal{B}$, where $\mathcal{B}$ is any triangular differential graded $S$-bocs. We start with some simple considerations on the additivity of the category TGMod- $\mathcal{B}$.

Lemma 6.1. Given a graded $S$-bocs $\mathcal{B}=(C, \mu, \epsilon)$, there is a functor of graded $k$-categories

$$
L=L_{\mathcal{B}}: \text { GMod }-S \longrightarrow \text { GMod- } \mathcal{B}
$$

which acts as the identity on objects and for any homogeneous morphism $f$ : $M \longrightarrow N$ of right graded $S$-modules, by definition

$$
L(f)=\left[M \otimes_{S} C \stackrel{i d_{M} \otimes \epsilon}{\longrightarrow} M \otimes_{S} S \cong M \stackrel{f}{\longrightarrow} N\right] .
$$

As a consequence, GMod- $\mathcal{B}$ is an additive category. When $\epsilon$ is surjective, the functor $L$ is faithful and we call it the canonical embedding.

Remark 6.2. If $\sigma_{j}: M_{j} \longrightarrow \bigoplus_{i=1}^{n} M_{i}$ and $\pi_{j}: \bigoplus_{i=1}^{n} M_{i} \longrightarrow M_{j}$ denote, respectively, the injection and projection maps corresponding to the direct sum $M=\bigoplus_{i=1}^{n} M_{i}$ in GMod- $S$, we have that $\underline{\sigma}_{j}:=L\left(\sigma_{j}\right): M_{j} \longrightarrow \bigoplus_{i=1}^{n} M_{i}$ and $\underline{\pi}_{j}:=L\left(\pi_{j}\right): \bigoplus_{i=1}^{n} M_{i} \longrightarrow M_{j}$ are, respectively, the injection and projection morphisms corresponding to the direct sum $\bigoplus_{i=1}^{n} M_{i}$ in GMod- $\mathcal{B}$. We will work with the usual matrix notation $\left(g_{j, i}\right)$ for a morphism $g: \bigoplus_{i=1}^{m} M_{i} \longrightarrow \bigoplus_{j=1}^{n} N_{j}$ in an additive category, the context will always permit the reader to avoid confusion with this notation: in GMod- $\mathcal{B}$, this means that $g_{j, i}=\underline{\pi}_{j} * g * \underline{\sigma_{i}}$ and, therefore, $g=\sum_{i, j} \underline{\sigma}_{j} * g_{j, i} * \underline{\pi}_{i}$.

Notice also that, for a differential graded $S$-bocs $\mathcal{B}$ with differential $\delta$, from the definition of the differential $\widehat{\delta}$ on the category GMod- $\mathcal{B}$ and (2.2), we immediately obtain that $\widehat{\delta}(L(h))=0$ for any morphism $h: M \longrightarrow N$ in GMod- $S$. 
Moreover, if $\mathcal{B}$ is a differential graded $S$-bocs, the category TGMod- $\mathcal{B}$ is additive too: given $\left(M_{1}, u_{1}\right),\left(M_{2}, u_{2}\right) \in$ TGMod- $\mathcal{B}$, their direct sum is

$$
\left(M_{1} \oplus M_{2}, u\right) \text {, where } u=\left(\begin{array}{cc}
u_{1} & 0 \\
0 & u_{2}
\end{array}\right) \in \operatorname{Hom}_{\mathrm{GMod}-\mathcal{B}}^{1}\left(M_{1} \oplus M_{2}, M_{1} \oplus M_{2}\right) .
$$

The injections are the morphisms $\underline{\sigma}_{i}:\left(M_{i}, u_{i}\right) \longrightarrow\left(M_{1} \oplus M_{2}, u\right)$, while the projections are the morphisms $\underline{\pi}_{i}:\left(M_{1} \oplus M_{2}, u\right) \longrightarrow\left(M_{i}, u_{i}\right)$. Indeed, since $u=\underline{\sigma}_{1} * u_{1} * \underline{\pi}_{1}+\underline{\sigma}_{2} * u_{2} * \underline{\pi}_{2}$, we obtain $u * \underline{\sigma}_{i}=\underline{\sigma}_{i} * u_{i} * \underline{\pi}_{i} * \underline{\sigma}_{i}=\underline{\sigma}_{i} * u_{i}$. Therefore, $\widehat{\delta}\left(\underline{\sigma}_{i}\right)+u * \underline{\sigma}_{i}-\underline{\sigma}_{i} * u_{i}=0$. Similarly, we have $\widehat{\delta}\left(\underline{\pi}_{i}\right)+u_{i} * \underline{\pi}_{i}-\underline{\pi}_{i} * u=0$.

Given a morphism $g: \bigoplus_{i=1}^{m} M_{i} \longrightarrow \bigoplus_{j=1}^{n} N_{j}$ in GMod- $\mathcal{B}$, with associated matrix $\left(g_{j, i}\right)$, from Leibniz rule, we have $\widehat{\delta}(g)=\sum_{i, j} \underline{\sigma}_{j} * \widehat{\delta}\left(g_{j, i}\right) * \underline{\pi}_{i}$. Then, we have $\widehat{\delta}(g)_{t, s}=\underline{\pi}_{t} * \widehat{\delta}(g) * \underline{\sigma}_{s}=\underline{\pi}_{t} * \underline{\sigma}_{t} * \widehat{\delta}\left(g_{t, s}\right) * \underline{\pi}_{s} * \underline{\sigma}_{s}=\widehat{\delta}\left(g_{t, s}\right)$, which means that the image under $\widehat{\delta}$ of the matrix $\left(g_{j, i}\right)$ is the matrix of images $\left(\widehat{\delta}\left(g_{j, i}\right)\right)$.

Lemma 6.3. Let $\mathcal{B}=(C, \mu, \epsilon, \delta)$ be a differential graded $S$-bocs. Assume thate : $(M, u) \longrightarrow(M, u)$ is an idempotent morphism in the category TGMod $^{0}-\mathcal{B}$ such that the idempotent $e: M \longrightarrow M$ splits in $\mathrm{GMod}^{0}-\mathcal{B}$, then e splits in $\mathrm{TGMod}^{0}-\mathcal{B}$.

Proof. By assumption, there is an isomorphism $h: M \longrightarrow M_{1} \oplus M_{2}$ in $\operatorname{GMod}^{0}-\mathcal{B}$ such that the following diagram commutes in $\operatorname{GMod}^{0}-\mathcal{B}$

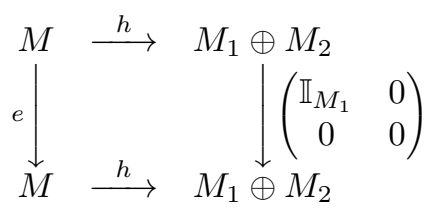

where $M_{1} \oplus M_{2}$ is the direct sum of graded right $\mathcal{B}$-modules.

According to (4.11), there is some $v \in \operatorname{Hom}_{\mathrm{GMod}-\mathcal{B}}^{1}\left(M_{1} \oplus M_{2}, M_{1} \oplus M_{2}\right)$ such that $h:(M, u) \longrightarrow\left(M_{1} \oplus M_{2}, v\right)$ is an isomorphism in TGMod ${ }^{0}-\mathcal{B}$. Then, $h * e * h^{-1}:\left(M_{1} \oplus M_{2}, v\right) \longrightarrow\left(M_{1} \oplus M_{2}, v\right)$ is a morphism in TGMod ${ }^{0}-\mathcal{B}$. Thus, $\widehat{\delta}\left(h * g * h^{-1}\right)+v *\left(h * e * h^{-1}\right)-\left(h * e * h^{-1}\right) * v=0$. But $\widehat{\delta}\left(h * e * h^{-1}\right)=$ $\widehat{\delta}\left(\begin{array}{cc}\mathbb{I}_{M_{1}} & 0 \\ 0 & 0\end{array}\right)=0$, because $\widehat{\delta}\left(\mathbb{I}_{M_{1}}\right)=0$. Hence, $\left(h * e * h^{-1}\right) * v=v *\left(h * e * h^{-1}\right)$. It follows that

$v=\left(\begin{array}{cc}v_{1} & 0 \\ 0 & v_{2}\end{array}\right)$, with $v_{1} \in \operatorname{Hom}_{\mathrm{GMod}-\mathcal{B}}^{1}\left(M_{1}, M_{1}\right)$ and $v_{2} \in \operatorname{Hom}_{\mathrm{GMod}-\mathcal{B}}^{1}\left(M_{2}, M_{2}\right)$.

Then, from the equality $\widehat{\delta}(v)+v * v=0$, we obtain the equalities

$$
\widehat{\delta}\left(v_{1}\right)+v_{1} * v_{1}=0 \text { and } \widehat{\delta}\left(v_{2}\right)+v_{2} * v_{2}=0 .
$$

Then, $\left(M_{1}, v_{1}\right)$ and $\left(M_{2}, v_{2}\right)$ belong to TGMod- $\mathcal{B}$, and

$$
h:(M, u) \longrightarrow\left(M_{1}, v_{1}\right) \oplus\left(M_{2}, v_{2}\right)
$$

is an isomorphism in $\operatorname{TGMod}^{0}-\mathcal{B}$ with $h * e * h^{-1}=\left(\begin{array}{cc}\mathbb{I}_{M_{1}} & 0 \\ 0 & 0\end{array}\right)$, thus $e$ splits in TGMod $^{0}-\mathcal{B}$, as claimed. 
For the sake of computational simplicity, it is convenient to rewrite the morphisms of the category GMod- $\mathcal{B}$ in a slightly different way.

Definition 6.4. Given a normal graded $S$-bocs $\mathcal{B}=(C, \mu, \epsilon)$, we can consider the following category GModI- $\mathcal{B}$. Its objects are the graded right $S$-modules and, for $d \in \mathbb{Z}$, a morphism $f: M \longrightarrow N$ of degree $d$ is a pair $f=\left(f^{0}, f^{1}\right)$, where $f^{0}: M \longrightarrow N$ and $f^{1}: \bar{C} \longrightarrow \operatorname{Hom}_{\mathrm{GMod}-k}(M, N)$ are homogeneous morphisms, $f^{0}$ of right $S$-modules and $f^{1}$ of $S$-S-bimodules, of degree $d$. Thus, the hom spaces are given by

$\operatorname{Hom}_{\mathrm{GModI}-\mathcal{B}}^{d}(M, N)=\operatorname{Hom}_{\mathrm{GMod}-S}^{d}(M, N) \times \operatorname{Hom}_{\mathrm{GMod}-S-S}^{d}\left(\bar{C}, \operatorname{Hom}_{\mathrm{GMod}-k}(M, N)\right)$.

The composition in this category is transfered from the composition in the category GMod- $\mathcal{B}$ with the help of the natural isomorphism

$$
\eta: \operatorname{Hom}_{\mathrm{GMod}-S}(M \otimes \bar{C}, N) \longrightarrow \operatorname{Hom}_{\mathrm{GMod}-S-S}\left(\bar{C}, \operatorname{Hom}_{\mathrm{GMod}-k}(M, N)\right) .
$$

So, the composition of two morphisms $f: M \longrightarrow N$ and $g: N \longrightarrow L$ in GModI- $\mathcal{B}$ is given by $g * f=\left((g * f)^{0},(g * f)^{1}\right)$, where $(g * f)^{0}=g^{0} f^{0}$ and the morphism of graded $S$-S-bimodules $(f * g)^{1}: \bar{C} \longrightarrow \operatorname{Hom}_{\mathrm{GMod}-k}^{*}(M, N)$ is given by

$$
(g * f)^{1}(c)=g^{1}(c) f^{0}+g^{0} f^{1}(c)+\sum_{i} g^{1}\left(c_{i}^{2}\right) f^{1}\left(c_{i}^{1}\right), \text { where } \bar{\mu}(c)=\sum_{i} c_{i}^{1} \otimes c_{i}^{2} .
$$

It is easy to show that the bijection $\left(f^{0}, f^{1}\right) \mapsto\left(f^{0}, \eta\left(f^{1}\right)\right)$ determines an isomorphism of categories GMod- $\mathcal{B} \longrightarrow$ GModI- $\mathcal{B}$. The formula for the composition of morphisms in GModI- $\mathcal{B}$ is the translation of the composition in GMod- $\mathcal{B}$ using the precedent bijection.

Lemma 6.5. For any graded triangular $S$-bocs $\mathcal{B}$, idempotents split in $\mathrm{GMod}^{0}-\mathcal{B}$.

Proof. In this proof, for the sake of notational simplicity, we write $g f$ instead of $g * f$ to indicate the composite morphism in $\mathrm{GM}^{0} \mathrm{II}^{0}-\mathcal{B}$.

It will be enough to show that any idempotent morphism $e=\left(e^{0}, e^{1}\right)$ : $M \longrightarrow M$ splits in GModI ${ }^{0}-\mathcal{B}$. Since idempotents clearly split in GMod- $S$, it will be enough to show that there is an isomorphism $h: M \longrightarrow M$ such that $\left(h e h^{-1}\right)^{1}=0$. Adopt the notation of (4.6), make $\bar{C}_{-1}:=0$, and let us first show the following.

Claim 1: There is a sequence of isomorphisms $M \stackrel{h_{1}}{\longrightarrow} M \stackrel{h_{2}}{\longrightarrow} M \stackrel{h_{3}}{\longrightarrow} \cdots$ in $\mathrm{GModI}^{0}-\mathcal{B}$ such that, for each $i \geq 1$, we have

1. Each isomorphism has the form $h_{i}=\left(i d_{M}, h_{i}^{1}\right)$;

2. $h_{i}^{1}\left(\bar{C}_{i-2}\right)=0$; and

3. For $e_{i}:=h_{i} \cdots h_{2} h_{1} e h_{1}^{-1} h_{2}^{-1} \cdots h_{i}^{-1}$, we have $e_{i}^{1}\left(\bar{C}_{i-1}\right)=0$.

Proof of the Claim 1: The inductive argument is essentially the same as the one given in 2] (5.12), but we recall it for the sake of the reader. At the base 
of the induction, we have the isomorphism $h_{1}=\left(i d_{M}, 0\right): M \longrightarrow M$ such that $h_{1}^{1}\left(\bar{C}_{-1}\right)=0$ and the idempotent $e_{1}:=e$ such that $e_{1}^{1}\left(\bar{C}_{0}\right)=0$.

Assume that we have constructed the isomorphisms $h_{1}, \ldots, h_{i}$ satisfying conditions 1-3. Notice that $e_{i}$ is idempotent, and so is $e_{i}^{0}$. From $e_{i}^{1}\left(\bar{C}_{i-1}\right)=0$ and the triangularity we obtain, for $c \in \bar{C}_{i}$ that $e_{i}^{1}(c)=\left(e_{i}^{2}\right)^{1}(c)=e_{i}^{0} e_{i}^{1}(c)+$ $e_{i}^{1}(c) e_{i}^{0}$. Hence, $e_{i}^{0} e_{i}^{1}(c) e_{i}^{0}=0$. Now, for $c \in \bar{C}$ define $h_{i+1}^{1}(c):=e_{i}^{1}(c) f_{i}^{0}$, where $f_{i}^{0} \in \operatorname{Hom}_{\text {GMod- } S}(M, M)$ will be specified in a moment. Then, clearly $h_{i+1}^{1}\left(\bar{C}_{i-1}\right)=0$ and $h_{i+1}^{1} \in \operatorname{Hom}_{\mathrm{GMod}-S-S}\left(\bar{C}, \operatorname{Hom}_{\mathrm{GMod}-k}(M, M)\right)$. Then, the pair $h_{i+1}=\left(1_{M}, h_{i+1}^{1}\right): M \longrightarrow M$ is a morphism in GModI ${ }^{0}-\mathcal{B}$. From (4.8), we know that $h_{i+1}$ is an isomorphism in $\mathrm{GModI}^{0}-\mathcal{B}$.

Let $g_{i+1}:=h_{i+1}^{-1}: M \longrightarrow M$. Then, $g_{i+1}^{0}=1_{M}$ and, since $\left(g_{i+1} h_{i+1}\right)^{1}=0$, we obtain for $c \in \bar{C}_{i}$ that $g_{i+1}^{1}(c)=-h_{i+1}^{1}(c)$. Then, by triangularity, we have for $c \in \bar{C}_{i}$,

$$
\begin{aligned}
\left(h_{i+1} e_{i} g_{i+1}\right)^{1}(c) & =h_{i+1}^{0}\left(e_{i} g_{i+1}\right)^{1}(c)+h_{i+1}^{1}(c)\left(e_{i} g_{i+1}\right)^{0} \\
& =e_{i}^{0} g_{i+1}^{1}(c)+e_{i}^{1}(c)+h_{i+1}^{1}(c) e_{i}^{0} \\
& =-e_{i}^{0} e_{i}^{1}(c) f_{i}^{0}+e_{i}^{1}(c)+e_{i}^{1}(c) f_{i}^{0} e_{i}^{0} \\
& =-e_{i}^{0} e_{i}^{1}(c) f_{i}^{0}+e_{i}^{0} e_{i}^{1}(c)+e_{i}^{1}(c) e_{i}^{0}+e_{i}^{1}(c) f_{i}^{0} e_{i}^{0} .
\end{aligned}
$$

Since $e_{i}^{0} e_{i}^{1}(c) e_{i}^{0}=0$, choosing $f_{i}^{0}:=1_{M}-2 e_{i}^{0}$ we obtain the equality $e_{i+1}^{1}(c)=$ $\left(h_{i+1} e_{i} g_{i+1}\right)^{1}(c)=0$, as we wanted.

From (1) and (2), we obtain, for $i \geq 1$ and $c \in \bar{C}_{i-1}$, the equality

$$
\left(h_{i+1} \cdots h_{2} h_{1}\right)^{1}(c)=\left(h_{i} \cdots h_{2} h_{1}\right)^{1}(c) .
$$

Indeed, this is clear for $i=1$. For $i \geq 2$, we have $\left(h_{i} \cdots h_{2} h_{1}\right)^{0}=i d_{M}$ and $h_{i+1}^{0}=i d_{M}$. By assumption, $\bar{\mu}(c)=\sum_{t} c_{t}^{1} \otimes c_{t}^{2}$, with $c_{t}^{1}, c_{t}^{2} \in \bar{C}_{i-2}$. Then, we obtain

$$
\begin{aligned}
\left(h_{i+1} \cdots h_{2} h_{1}\right)^{1}(c)= & \left(h_{i+1}\left(h_{i} \cdots h_{2} h_{1}\right)\right)^{1}(c) \\
= & h_{i+1}^{1}(c)+\left(h_{i} \cdots h_{2} h_{1}\right)^{1}(c) \\
& +\sum_{t} h_{i+1}^{1}\left(c_{t}^{2}\right)\left(h_{i} \cdots h_{2} h_{1}\right)^{1}\left(c_{t}^{1}\right) \\
= & \left(h_{i} \cdots h_{2} h_{1}\right)^{1}(c) .
\end{aligned}
$$

Then, we can consider the map $h^{1}: \bar{C} \longrightarrow \operatorname{Hom}_{\mathrm{GMod}-k}(M, M)$ defined by

$$
h^{1}(c)=\left(h_{i} \cdots h_{2} h_{1}\right)^{1}(c), \text { for } c \in \bar{C}_{i-1} .
$$

Since $h^{1}$ is a morphism of graded $S$-S-bimodules, we can consider the isomorphism $h=\left(i d_{M}, h^{1}\right): M \longrightarrow M$ in $\mathrm{GModI}^{0}-\mathcal{B}$.

The statement (4.7) gives us in this context that any morphism of the form $g=\left(0, g^{1}\right): M \longrightarrow M$ satisfies that for any $i \geq 1$ there is some $n_{i} \in \mathbb{N}$ such that for $c \in \bar{C}_{i}$ and $n \geq n_{i}$ we have $\left(g^{n}\right)^{1}(c)=0$. So, we have a well defined morphism $\sum_{n=0}^{\infty} g^{n}: M \longrightarrow M$. So, consider $g:=\left(0,-h^{1}\right)$ and notice that $h=\mathbb{I}_{M}-g$, so $h^{-1}=\sum_{n=0}^{\infty} g^{n}=\mathbb{I}_{M}+\sum_{n=1}^{\infty} g^{n}$. In particular, $\left(h^{-1}\right)^{1}=\sum_{n=1}^{\infty}\left[\left(0,-h^{1}\right)^{n}\right]^{1}$.

Consider the iterated comultiplication $\bar{\mu}^{n}: \bar{C} \longrightarrow \bar{C}^{\otimes(n+1)}$, defined recursively, for $n \geq 1$, by $\bar{\mu}^{0}=i d_{\bar{C}}$ and $\bar{\mu}^{n}=\left(i d_{\bar{C}} \otimes \bar{\mu}^{(n-1)}\right) \bar{\mu}$.

Claim 2: Given any morphism of the form $g=\left(0, g^{1}\right): M \longrightarrow M$ in $\mathrm{GM} \mathrm{dI}^{0}-\mathcal{B}$, the following holds 
1. For $n \geq 1$ and $c \in \bar{C}$, we have

$$
\left(g^{n}\right)^{1}(c)=\sum_{t} g^{1}\left(c_{t}^{n}\right) \cdots g^{1}\left(c_{t}^{1}\right), \text { where } \bar{\mu}^{(n-1)}(c)=\sum_{t} c_{t}^{1} \otimes \cdots \otimes c_{t}^{n} .
$$

2. Moreover, for $n \geq 2, i \geq 1$, and $c_{i} \in \bar{C}_{i}$, we have $c_{t}^{1}, \ldots, c_{t}^{n} \in \bar{C}_{i-1}$.

Proof of the Claim 2: We proceed by induction. Item (1) holds trivially for $n=1$. So assume item (1) holds for $n$. Then, for $c \in \bar{C}$, we have

$$
\begin{aligned}
\bar{\mu}^{n}(c) & =\left(i d_{\bar{C}} \otimes \bar{\mu}^{(n-1)}\right) \bar{\mu}(c) \\
& =\left(i d_{\bar{C}} \otimes \bar{\mu}^{(n-1)}\right)\left(\sum_{s} c_{s}^{1} \otimes c_{s}^{2}\right) \\
& =\sum_{s} c_{s}^{1} \otimes \bar{\mu}^{(n-1)}\left(c_{s}^{2}\right) \\
& =\sum_{s} \sum_{t_{s}} c_{s}^{1} \otimes c_{t_{s}}^{2} \otimes \cdots \otimes c_{t_{s}}^{n+1}
\end{aligned}
$$

where $\bar{\mu}(c)=\sum_{s} c_{s}^{1} \otimes c_{s}^{2}$ and, for each $s, \bar{\mu}^{(n-1)}\left(c_{s}^{2}\right)=\sum_{t_{s}} c_{t_{s}}^{2} \otimes \cdots \otimes c_{t_{s}}^{n+1}$. Thus,

$$
\left(g^{n+1}\right)^{1}(c)=\sum_{s}\left(g^{n}\right)^{1}\left(c_{s}^{2}\right) g^{1}\left(c_{s}^{1}\right)=\sum_{s} \sum_{t_{s}} g^{1}\left(c_{t_{s}}^{n+1}\right) \cdots g^{1}\left(c_{t_{s}}^{2}\right) g^{1}\left(c_{s}^{1}\right) .
$$

Now, if $n \geq 2, i \geq 1$, and $c \in \bar{C}_{i}$, we have from the triangularity condition that $c_{s}^{1} \in \bar{C}_{i-1}$. We also have that $c_{t_{s}}^{2}, \ldots, c_{t_{s}}^{n+1} \in \bar{C}_{i-1}$ by the induction hypothesis for item (2). The Claim 2 is proved.

Assume that $n \geq 1, c \in \bar{C}_{i-1}$, and $\bar{\mu}^{(n-1)}(c)=\sum_{t} c_{t}^{1} \otimes \cdots \otimes c_{t}^{n}$, then

$$
\begin{aligned}
{\left[\left(0,-h^{1}\right)^{n}\right]^{1}(c) } & =(-1)^{n} \sum_{t} h^{1}\left(c_{t}^{n}\right) \cdots h^{1}\left(c_{t}^{1}\right) \\
& =(-1)^{n} \sum_{t}\left(h_{i} \cdots h_{1}\right)^{1}\left(c_{t}^{n}\right) \cdots\left(h_{i} \cdots h_{1}\right)^{1}\left(c_{t}^{1}\right) \\
& =\left[\left(0,-\left(h_{i} \cdots h_{1}\right)^{1}\right)^{n}\right]^{1}(c),
\end{aligned}
$$

and

$\left(h^{-1}\right)^{1}(c)=\sum_{n=1}^{\infty}\left[\left(0,-h^{1}\right)^{n}\right]^{1}(c)=\sum_{n=1}^{\infty}\left[\left(0,-\left(h_{i} \cdots h_{1}\right)^{1}\right)^{n}\right]^{1}(c)=\left[\left(h_{i} \cdots h_{1}\right)^{-1}\right]^{1}(c)$.

Claim 3: Let $p, q: M \longrightarrow M$ be any morphisms in $\mathrm{GModI}^{0}-\mathcal{B}$. Then,

1. Given a family of morphisms $\left\{p_{i}: M \longrightarrow M\right\}_{i \geq 1}$ in $\mathrm{GModI}^{0}-\mathcal{B}$ such that $p_{i}^{0}=p^{0}$ and $p_{i}^{1}(c)=p^{1}(c)$, for $i \geq 1$ and $c \in \bar{C}_{i-1}$, we have

$$
\left(q p_{i}\right)^{1}(c)=(q p)^{1}(c), \text { for } c \in \bar{C}_{i-1} .
$$

2. Given a family of morphisms $\left\{q_{i}: M \longrightarrow M\right\}_{i \geq 1}$ in $\operatorname{GModI}^{0}-\mathcal{B}$ such that $q_{i}^{0}=q^{0}$ and $q_{i}^{1}(c)=q^{1}(c)$, for $i \geq 1$ and $c \in \bar{C}_{i-1}$, we have

$$
\left(q_{i} p\right)^{1}(c)=(q p)^{1}(c), \text { for } c \in \bar{C}_{i-1} .
$$


Proof of Claim 3: (1): For $c \in \bar{C}_{i-1}$, we have $\bar{\mu}(c)=\sum_{t} c_{t}^{1} \otimes c_{t}^{2}$, with $c_{t}^{1}, c_{t}^{2} \in \bar{C}_{i-1}$, so

$$
\begin{aligned}
(q p)^{1}(c) & =q^{0} p^{1}(c)+q^{1}(c) p^{0}+\sum_{t} q^{1}\left(c_{t}^{2}\right) p^{1}\left(c_{t}^{1}\right) \\
& =q^{0} p_{i}^{1}(c)+q^{1}(c) p_{i}^{0}+\sum_{t} q^{1}\left(c_{t}^{2}\right) p_{i}^{1}\left(c_{t}^{1}\right) \\
& =\left(q p_{i}\right)^{1}(c)
\end{aligned}
$$

The proof of (2) is similar.

Then, for $c \in \bar{C}$, say with $c \in \bar{C}_{i-1}$, we have

$$
\left(h e h^{-1}\right)^{1}(c)=\left(h_{i} \cdots h_{1} e h^{-1}\right)^{1}(c)=\left(h_{i} \cdots h_{1} e\left(h_{i} \cdots h_{1}\right)^{-1}\right)^{1}(c)=e_{i}^{1}(c)=0 .
$$

Therefore, $\left(h e h^{-1}\right)^{1}=0$, as we wanted to show.

Definition 6.6. Let $\mathcal{B}=(C, \mu, \epsilon)$ be a graded $S$-bocs. A graded right $\mathcal{B}$ comodule $\left(M, \mu_{M}\right)$ is called induced iff it is isomorphic to a right $\mathcal{B}$-comodule of the form $\operatorname{Ind}_{\mathcal{B}}(N)=\left(N \otimes C, i d_{N} \otimes \mu\right)$ for some $N \in$ GMod- $S$. We denote by $\mathrm{GCoind}-\mathcal{B}$ the full subcategory of GComod- $\mathcal{B}$ formed by the induced $\mathcal{B}$ comodules. So GCoind- $\mathcal{B}$ is an additive subcategory of GComod- $\mathcal{B}$.

If $\mathcal{B}=(C, \mu, \epsilon, \delta)$ is a differential graded $S$-bocs, a differential graded right $\mathcal{B}$ comodule $\left(M, \mu_{M}, \delta_{M}\right)$ is called induced iff $\left(M, \mu_{M}\right) \in$ GCoind- $\mathcal{B}$. The full subcategory of DGComod- $\mathcal{B}$ formed by the differential graded induced $\mathcal{B}$-comodules will be denoted by DGCoind- $\mathcal{B}$. So, DGCoind- $\mathcal{B}$ is an additive subcategory of DGComod- $\mathcal{B}$.

Here again the superindex 0 in $\mathrm{GCoind}^{0}-\mathcal{B}$ and $\mathrm{DGCoind}^{0}-\mathcal{B}$ indicates the subcategory with all objects and only degree zero morphisms.

Proposition 6.7. Let $\mathcal{B}=(C, \mu, \epsilon, \delta)$ be a triangular differential graded $S$-bocs . Then, in the categories $\operatorname{GMod}^{0}-\mathcal{B}, \mathrm{GCoind}^{0}-\mathcal{B}$, $\mathrm{TGMod}^{0}-\mathcal{B}$, and $\mathrm{DGCoind}^{0}-\mathcal{B}$ idempotents split.

Proof. From (6.5), idempotents split in $\operatorname{GMod}^{0}-\mathcal{B}$. But $\operatorname{GMod}^{0}-\mathcal{B}$ is equivalent to this category, so idempotents split in $\mathrm{GMod}^{0}-\mathcal{B}$. By (6.3), idempotents split in TGMod $^{0}-\mathcal{B}$,

By (3.4), we know that the categories $\operatorname{GMod}^{0}-\mathcal{B}$ and $\mathrm{GCoind}^{0}-\mathcal{B}$ are equivalent categories, and that $\operatorname{TGMod}^{0}-\mathcal{B}$ and $\mathrm{DGCoind}^{0}-\mathcal{B}$ are equivalent categories, so idempotents split in $\mathrm{GCoind}^{0}-\mathcal{B}$ and in $\mathrm{DGCoind}^{0}-\mathcal{B}$.

Remark 6.8. Given a triangular graded $S$-bocs $\mathcal{B}$, we have the equivalent categories GMod- $\mathcal{B} \simeq$ GMod- $\mathcal{B} \simeq$ GModI- $\mathcal{B}$. Then, we have canonical embeddings GMod- $S \longrightarrow$ GMod- $\mathcal{B}$ and GMod- $S \longrightarrow$ GModI- $\mathcal{B}$ which we denote with the same symbol $L$, as in (6.1).

Notice that given $h: M \longrightarrow N$ in GMod- $S$, we have $L(h)=(h, 0)$ in GMod- $\mathcal{B}$ and in GModI- $\mathcal{B}$.

The following statement can be proved as in [2](6.2). 
Lemma 6.9. Let $\mathcal{B}$ be a triangular graded $S$-bocs. Then, any exact sequence

$$
0 \longrightarrow M \stackrel{f^{0}}{\longrightarrow} E \stackrel{g^{0}}{\longrightarrow} N \longrightarrow 0
$$

in GMod-S determines an exact pair $M \stackrel{\left(f^{0}, 0\right)}{\longrightarrow} E \stackrel{\left(g^{0}, 0\right)}{\longrightarrow} N$ in GModI- $\mathcal{B}$.

Lemma 6.10. Let $\mathcal{B}$ be a triangular graded $S$-bocs. Then, for any morphism $f=\left(f^{0}, f^{1}\right): M \longrightarrow N$ in GMod $^{0}-\mathcal{B}$ the following holds.

1. If $f^{0}$ is surjective, there is an automorphism $h$ of $M$ such that $(f * h)^{1}=0$.

2. If $f^{0}$ is injective, there is an automorphism $h$ of $N$ such that $(h * f)^{1}=0$.

Proof. We will prove only (1). Assume that $f^{0}: M \longrightarrow N$ is surjective in GMod- $S$. Since $S$ is semisimple, $f^{0}$ is a retraction. Choose a right inverse $t^{0}: N \longrightarrow M$ for $f^{0}$. Adopt the notation of (4.6), skip the star in the notation for the composition in $\operatorname{GModI}^{0}-\mathcal{B}$, and make $\bar{C}_{-1}=0$. We first show the following.

Claim: There is a sequence of isomorphisms $\cdots \stackrel{h_{3}}{\longrightarrow} M \stackrel{h_{2}}{\longrightarrow} M \stackrel{h_{1}}{\longrightarrow} M$ such that, for each $i \geq 1$, we have

1. Each isomorphism has the form $h_{i}=\left(i d_{M}, h_{i}^{1}\right)$;

2. $h_{i}^{1}\left(\bar{C}_{i-2}\right)=0$; and

3. For $f_{i}:=f h_{1} h_{2} \cdots h_{i}$, we have $f_{i}^{1}\left(\bar{C}_{i-1}\right)=0$.

Proof of the Claim. The inductive argument is essentially the same as the one given in [2] (5.7), but we rephrase it here for the sake of the reader. At the base of the induction, we have the isomorphism $h_{1}=\left(i d_{M}, 0\right): M \longrightarrow M$ such that $h_{1}^{1}\left(\bar{C}_{-1}\right)=0$ and the morphism $f_{1}=f$ is such that $f_{1}^{1}\left(\bar{C}_{0}\right)=0$.

Assume that we have constructed the isomorphisms $h_{1}, \ldots, h_{i}$ satisfying conditions 1-3 and define $h_{i+1}^{1}(c):=-t^{0} f_{i}^{1}(c)$, for $c \in \bar{C}$.

Clearly $h_{i+1}^{1}\left(\bar{C}_{i-1}\right)=0$ and $h_{i+1}^{1} \in \operatorname{Hom}_{\mathrm{GMod}-S-S}^{0}\left(\bar{C}, \operatorname{Hom}_{\mathrm{GMod}-k}(M, M)\right)$. Then, the pair $h_{i+1}=\left(i d_{M}, h_{i+1}^{1}\right): M \longrightarrow M$ is a morphism in GModI ${ }^{0}-\mathcal{B}$. From (4.8), we know that $h_{i+1}$ is an isomorphism in $\mathrm{GModI}^{0}-\mathcal{B}$. Moreover, for $c \in \bar{C}_{i}$, we have $\bar{\mu}(c)=\sum_{t} c_{t}^{1} \otimes c_{t}^{2}$, with $c_{t}^{1}, c_{t}^{2} \in \bar{C}_{i-1}$. Notice also that $f_{i}^{0}=\left(f h_{1} \cdots h_{i}\right)^{0}=f^{0}$ and $h_{i+1}^{0}=i d_{M}$. Then, we have

$$
\begin{aligned}
f_{i+1}^{1}(c) & =\left(f_{i} h_{i+1}\right)^{1}(c) \\
& =f_{i}^{0} h_{i+1}^{1}(c)+f_{i}^{1}(c) h_{i+1}^{0}+\sum_{t} f_{i}^{1}\left(c_{t}^{2}\right) h_{i+1}^{1}\left(c_{t}^{1}\right) \\
& =-f^{0} t^{0} f_{i}^{1}(c)+f_{i}^{1}(c)=0
\end{aligned}
$$

as we wanted.

From (1) and (2), we obtain, for $i \geq 1$ and $c \in \bar{C}_{i-1}$, the equality

$$
\left(h_{1} h_{2} \cdots h_{i+1}\right)^{1}(c)=\left(h_{1} h_{2} \cdots h_{i}\right)^{1}(c) .
$$


Indeed, this is clear for $i=1$. For $i \geq 2$, we have $\left(h_{1} h_{2} \cdots h_{i}\right)^{0}=i d_{M}$ and $h_{i+1}^{0}=i d_{M}$. By assumption, $\bar{\mu}(c)=\sum_{t} c_{t}^{1} \otimes c_{t}^{2}$, with $c_{t}^{1}, c_{t}^{2} \in \bar{C}_{i-2}$. Then, we obtain

$$
\begin{aligned}
\left(h_{1} h_{2} \cdots h_{i+1}\right)^{1}(c)= & \left.\left(\left(h_{1} h_{2} \cdots h_{i}\right) h_{i+1}\right)\right)^{1}(c) \\
= & \left(h_{1} h_{2} \cdots h_{i}\right)^{1}(c)+h_{i+1}^{1}(c) \\
& +\sum_{t}\left(h_{1} h_{2} \cdots h_{i}\right)^{1}\left(c_{t}^{2}\right) h_{i+1}^{1}\left(c_{t}^{1}\right) \\
= & \left(h_{1} h_{2} \cdots h_{i}\right)^{1}(c) .
\end{aligned}
$$

Then, we can consider the map $h^{1}: \bar{C} \longrightarrow \operatorname{Hom}_{\mathrm{GMod}-k}(M, M)$ defined by

$$
h^{1}(c)=\left(h_{1} h_{2} \cdots h_{i}\right)^{1}(c) \text {, for } c \in \bar{C}_{i-1} .
$$

Since $h^{1}$ is a morphism of graded $S$-S-bimodules, we can consider the isomorphism $h=\left(i d_{M}, h^{1}\right): M \longrightarrow M$ in $\operatorname{GModI}^{0}-\mathcal{B}$. For $c \in \bar{C}$, say with $c \in \bar{C}_{i-1}$, we have

$$
(f h)^{1}(c)=\left(f h_{1} h_{2} \cdots h_{i}\right)^{1}(c)=f_{i}^{1}(c)=0 .
$$

Proposition 6.11. Let $\mathcal{B}$ be a triangular differential $S$-bocs. Denote by $\mathcal{E}_{0}$ the class of composable pairs $M \stackrel{f}{\longrightarrow} E \stackrel{g}{\longrightarrow} N$ in $\operatorname{GMod}^{0}-\mathcal{B}$ such that $g * f=0$ and

$$
0 \longrightarrow M \stackrel{f^{0}}{\longrightarrow} E \stackrel{g^{0}}{\longrightarrow} N \longrightarrow 0
$$

is an exact sequence in GMod-S. Then we have:

1. The pair $\left(\operatorname{GMod}^{0}-\mathcal{B}, \mathcal{E}_{0}\right)$ is an exact category. The class $\mathcal{E}_{0}$ consists of the split exact pairs in $\mathrm{GMod}^{0}-\mathcal{B}$.

2. A morphism $f: M \longrightarrow E$ in $\operatorname{GMod}^{0}-\mathcal{B}$ is an $\mathcal{E}_{0}$-inflation iff $f^{0}: M \longrightarrow E$ is injective.

3. A morphism $g: E \longrightarrow N$ in $\operatorname{GMod}^{0}-\mathcal{B}$ is an $\mathcal{E}_{0}$-deflation iff $g^{0}: E \longrightarrow N$ is surjective.

Proof. By (6.5), we already know in the category GModI ${ }^{0}-\mathcal{B}$ idempotents split. Follow the argument of the proof of 2 (6.6 and 6.7), using (6.9) and (6.10), to show that $\left(\operatorname{GMod}^{0}-\mathcal{B}, \mathcal{E}_{0}\right)$ is an exact category. From (4.10), (4.8), and (6.9) we get that any composable pair in $\mathcal{E}_{0}$ is a split exact pair in $\operatorname{GMod}^{0}-\mathcal{B}$.

\section{The Frobenius category of twisted modules}

Given a triangular differential graded $S$-bocs $\mathcal{B}=(C, \mu, \epsilon, \delta)$ we will describe a natural structure of a Frobenius category on $\mathrm{TGMod}^{0}-\mathcal{B}$, in the following sense.

Definition 7.1. Let $\mathcal{A}$ be an additive $k$-category where idempotents split, and let $\mathcal{E}$ be an exact structure on $\mathcal{A}$. Then $(\mathcal{A}, \mathcal{E})$ is called a Frobenius category iff it has enough $\mathcal{E}$-projectives and enough $\mathcal{E}$-injectives and, moreover, the class of $\mathcal{E}$-projectives coincides with the class of $\mathcal{E}$-injectives. 
Definition 7.2. Consider the class $\mathcal{E}$ of composable morphisms in $\mathrm{TGMod}^{0}-\mathcal{B}$

$$
(M, u) \stackrel{f}{\longrightarrow}(E, v) \stackrel{g}{\longrightarrow}(N, w)
$$

such that $g * f=0$ and the short sequence of first components

$$
0 \longrightarrow M \stackrel{f^{0}}{\longrightarrow} E \stackrel{g^{0}}{\longrightarrow} N \longrightarrow 0
$$

is an exact sequence in GMod-S. Equivalently, the composable pair

$$
M \stackrel{f}{\longrightarrow} E \stackrel{g}{\longrightarrow} N
$$

is a split exact pair in $\mathrm{GMod}^{0}-\mathcal{B}$.

Lemma 7.3. Any composable pair of morphisms in $\mathrm{TGMod}^{0}-\mathcal{B}$ of the form

$$
\left(M_{1}, u_{1}\right) \stackrel{s=\left(\mathbb{I}_{M_{1}}, 0\right)^{t}}{\longrightarrow}\left(M_{1} \oplus M_{2}, v\right) \stackrel{p=\left(0, \mathbb{I}_{M_{2}}\right)}{\longrightarrow}\left(M_{2}, u_{2}\right)
$$

belongs to $\mathcal{E}$ and is an exact pair in $\mathrm{TGMod}^{0}-\mathcal{B}$.

Proof. Clearly $M_{1} \stackrel{s}{\longrightarrow} M_{1} \oplus M_{2} \stackrel{p}{\longrightarrow} M_{2}$ is a split exact pair in $\mathrm{GMod}^{0}-\mathcal{B}$.

In order to show that $s$ is the kernel of $p$ in $\operatorname{TGMod}^{0}-\mathcal{B}$, take any morphism $t:(N, w) \longrightarrow\left(M_{1} \oplus M_{2}, v\right)$ in $\mathrm{TGM}^{0}-\mathcal{B}$ such that $p * t=0$. Then, there is a morphism $t^{\prime}: N \longrightarrow M_{1}$ in $\operatorname{GMod}^{0}-\mathcal{B}$ such that $t=s * t^{\prime}$.

We have $\widehat{\delta}(t)=\widehat{\delta}\left(s * t^{\prime}\right)=s * \widehat{\delta}\left(t^{\prime}\right)$. Since $s$ is a morphism in $\operatorname{TGMod}^{0}-\mathcal{B}$, we have $\widehat{\delta}(s)+v * s-s * u_{1}=v * s-s * u_{1}$, so $v * s=s * u_{1}$. We also have that $\widehat{\delta}(t)+v * t-t * w=0$. Then,

$$
\begin{aligned}
s *\left[\widehat{\delta}\left(t^{\prime}\right)+u_{1} * t^{\prime}-t^{\prime} * w\right] & =s * \widehat{\delta}\left(t^{\prime}\right)+s * u_{1} * t^{\prime}-s * t^{\prime} * w \\
& =\widehat{\delta}(t)+v * s * t^{\prime}-s * t^{\prime} * w \\
& =\widehat{\delta}(t)+v * t-t * w=0 .
\end{aligned}
$$

Since $s$ is a monomorphism in $\operatorname{GMod}^{0}-\mathcal{B}$, we obtain $\widehat{\delta}\left(t^{\prime}\right)+u_{1} * t^{\prime}-t^{\prime} * w=0$. So $t^{\prime}:(N, w) \longrightarrow\left(M_{1}, u_{1}\right)$ is a morphism in $\operatorname{TGMod}^{0}-\mathcal{B}$. Hence, $s$ is the kernel of $p$ in $\operatorname{TGMod}^{0}-\mathcal{B}$. The proof of the fact that the cokernel of $s$ is $p$ in $\operatorname{TGMod}^{0}-\mathcal{B}$ is dual.

Lemma 7.4. Let $\mathcal{B}$ be a triangular differential $S$-bocs. Then, we have:

1. A morphism $g:(M, u) \longrightarrow\left(M_{2}, u_{2}\right)$ in $\mathrm{TGMOd}^{0}-\mathcal{B}$ is an $\mathcal{E}$-deflation iff $g: M \longrightarrow M_{2}$ is a retraction in $\operatorname{GMod}^{0}-\mathcal{B}$.

2. A morphism $f:\left(M_{1}, u_{1}\right) \longrightarrow(M, u)$ in $\mathrm{TGMod}^{0}-\mathcal{B}$ is an $\mathcal{E}$-inflation iff $f: M_{1} \longrightarrow M$ is a section in $\mathrm{GMod}^{0}-\mathcal{B}$.

3. Every composable pair in $\mathcal{E}$ is an exact pair in $\mathrm{TGMod}^{0}-\mathcal{B}$. 
Proof. (1): Assume that $g: M \longrightarrow M_{2}$ is a retraction in $\mathrm{GM}^{0}-\mathcal{B}$. Since in $\mathrm{GMod}^{0}-\mathcal{B}$ idempotents split, the retraction $g$ has a kernel and, moreover, there is a commutative diagram in $\operatorname{GMod}^{0}-\mathcal{B}$

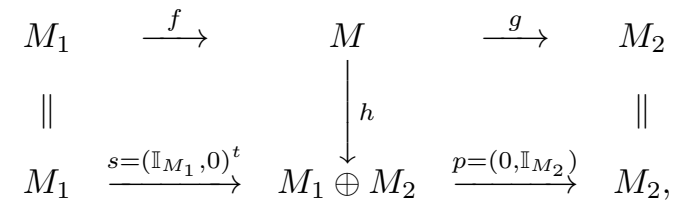

where $h: M \longrightarrow M_{1} \oplus M_{2}$ is an isomorphism. From (4.11), we know there is $v \in \operatorname{Hom}_{\text {GMod- } \mathcal{B}}^{1}\left(M_{1} \oplus M_{2}, M_{1} \oplus M_{2}\right)$ such that $h:(M, u) \longrightarrow\left(M_{1} \oplus M_{2}, v\right)$ is an isomorphism in TGMod ${ }^{0}-\mathcal{B}$. Therefore, $g * h^{-1}:\left(M_{1} \oplus M_{2}, v\right) \longrightarrow\left(M_{2}, u_{2}\right)$ is a morphism in TGMod ${ }^{0}-\mathcal{B}$, so $\widehat{\delta}\left(g * h^{-1}\right)+u_{2} *\left(g * h^{-1}\right)-\left(g * h^{-1}\right) * v=0$. Since $\widehat{\delta}\left(g * h^{-1}\right)=\widehat{\delta}(p)=0$, we obtain $u_{2} *\left(g * h^{-1}\right)=\left(g * h^{-1}\right) * v$. This implies that $v=\left(\begin{array}{cc}u_{1} & x \\ 0 & u_{2}\end{array}\right)$, where $u_{1} \in \operatorname{Hom}_{\mathrm{GMod}-\mathcal{B}}^{1}\left(M_{2}, M_{2}\right)$ and $x \in \operatorname{Hom}_{\mathrm{GMod}-\mathcal{B}}^{1}\left(M_{2}, M_{1}\right)$. Moreover, the equation $\widehat{\delta}(v)+v * v=0$ implies that $\left(M_{1}, u_{1}\right) \in$ TGMod- $\mathcal{B}$. Notice also that $s:\left(M_{1}, u_{1}\right) \longrightarrow\left(M_{1} \oplus M_{2}, v\right)$ is a morphism in $\operatorname{TGMod}^{0}-\mathcal{B}$, because

$$
\widehat{\delta}(s)+v * s-s * u_{1}=\left(\begin{array}{cc}
u_{1} & x \\
0 & u_{2}
\end{array}\right) *\left(\begin{array}{c}
\mathbb{I}_{M_{1}} \\
0
\end{array}\right)-\left(\begin{array}{c}
\mathbb{I}_{M_{1}} \\
0
\end{array}\right) * u_{1}=0 .
$$

Then, the composable pair $\left(M_{1}, u_{1}\right) \stackrel{f}{\longrightarrow}(M, u) \stackrel{g}{\longrightarrow}\left(M_{2}, u_{2}\right)$ in the category TGMod $^{0}-\mathcal{B}$ belongs to $\mathcal{E}$ and $g:(M, u) \longrightarrow\left(M_{2}, u_{2}\right)$ is an $\mathcal{E}$-deflation.

The proof of (2) is similar.

(3): Consider any composable pair $(L, w) \stackrel{f}{\longrightarrow}(M, u) \stackrel{g}{\longrightarrow}\left(M_{2}, u_{2}\right)$ in $\mathcal{E}$. Then, $g: M \longrightarrow M_{2}$ is a retraction in $\operatorname{GMod}^{0}-\mathcal{B}$, so we can apply the preceding argument to construct the following commutative diagram in $\operatorname{TGM}^{0}-\mathcal{B}$

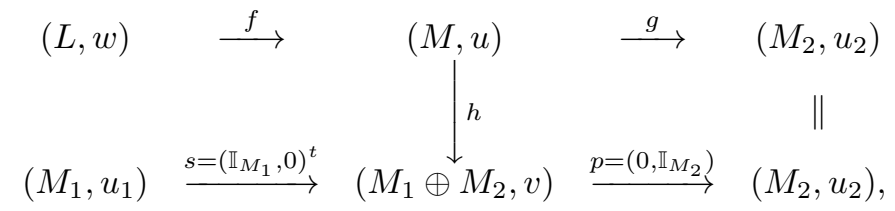

where $h$ is an isomorphism. From (7.3), we know that the second row is an exact pair in $\mathrm{TGMod}^{0}-\mathcal{B}$. Then, since $p * h * f=0$, there is a morphism $t:(L, w) \longrightarrow\left(M_{1}, u_{1}\right)$ in $\operatorname{TGMod}^{0}-\mathcal{B}$ such that $s * t=h * f$. If we consider the first components of the underlying diagram in GMod- $S$, we have the following commutative diagram where the vertical arrows are isomorphisms

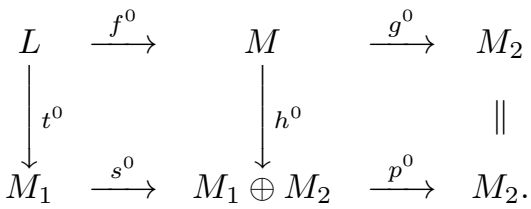

Hence $t:(L, w) \longrightarrow\left(M_{1}, u_{1}\right)$ is an isomorphism in $\mathrm{TGMod}^{0}-\mathcal{B}$. It follows that the composable pair $(L, w) \stackrel{f}{\longrightarrow}(M, u) \stackrel{g}{\longrightarrow}\left(M_{2}, u_{2}\right)$ is exact. 
Proposition 7.5. Let $\mathcal{B}$ be a triangular $S$-bocs, then we have the following.

1. The pair $\left(\operatorname{TGMod}^{0}-\mathcal{B}, \mathcal{E}\right)$ is an exact category.

2. A morphism $f:\left(M, u_{M}\right) \longrightarrow\left(E, u_{E}\right)$ in $\mathrm{TGMod}^{0}-\mathcal{B}$ is an $\mathcal{E}$-inflation iff $f^{0}: M \longrightarrow E$ is injective.

3. A morphism $g:\left(E, u_{E}\right) \longrightarrow\left(N, u_{N}\right)$ in $\mathrm{TGMod}^{0}-\mathcal{B}$ is an $\mathcal{E}$-deflation iff $g^{0}: E \longrightarrow N$ is surjective.

Proof. From the last section, we know that idempotents split in $\operatorname{TGMod}^{0}-\mathcal{B}$. The description of the $\mathcal{E}$-inflations and the $\mathcal{E}$-deflations follows from (7.4) and (6.11). The fact that $\mathcal{E}$ is a class of exact pairs closed under isomorphisms, follows from (7.4).

Consider a morphism $f: \underline{Z^{\prime}} \longrightarrow \underline{Z}$ and a deflation $d: \underline{Y} \longrightarrow \underline{Z}$ in TGMod ${ }^{0}-\mathcal{B}$. Consider the morphism $(f, d): \underline{Z^{\prime}} \oplus \underline{Y} \longrightarrow \underline{Z}$ in TGMod ${ }^{0}-\mathcal{B}$. Since $d: \underline{Y} \longrightarrow \underline{Z}$ is a deflation, the morphism $d: Y \longrightarrow Z$ is a retraction in $\operatorname{GMod}^{0}-\mathcal{B}$ and, therefore, so is $(f, d): Z^{\prime} \oplus Y \longrightarrow Z$. Then, $(f, d): \underline{Z}^{\prime} \oplus \underline{Y} \longrightarrow \underline{Z}$ is a deflation in $\mathrm{TGMod}^{0}-\mathcal{B}$ and it appears in an exact pair of $\mathcal{E}$

$$
\underline{Y}^{\prime} \stackrel{(\widehat{d},-\widehat{f})^{t}}{\longrightarrow} \underline{Z}^{\prime} \oplus \underline{Y} \stackrel{(f, d)}{\longrightarrow} \underline{Z} .
$$

Therefore, we have a pull-back diagram in the category TGMod $^{0}-\mathcal{B}$

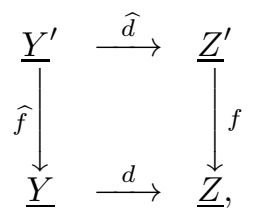

and an exact sequence in GMod- $S$ given by the first components

$$
Y^{\prime} \stackrel{\left(\widehat{d}^{0},-\widehat{f}^{0}\right)^{t}}{\longrightarrow} Z^{\prime} \oplus Y \stackrel{\left(f^{0}, d^{0}\right)}{\longrightarrow} Z .
$$

So we have the pullback-diagram in the category GMod- $S$

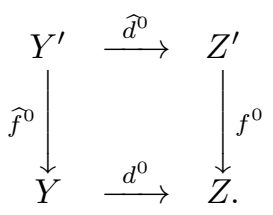

Since $d^{0}$ is surjective, then $\widehat{d}^{0}$ is surjective, thus $\widehat{d}: Y^{\prime} \longrightarrow Z^{\prime}$ is a retraction, and $\hat{d}: \underline{Y^{\prime}} \longrightarrow \underline{Z^{\prime}}$ is an $\mathcal{E}$-deflation.

The other requirements in the definition of an exact structure are easy to verify for $\mathcal{E}$ using (7.4).

We shall see that TGMod $^{0}-\mathcal{B}$ is a Frobenius category with the following additional structure. 
Definition 7.6. A Frobenius category $(\mathcal{A}, \mathcal{E})$ is called special iff there are an exact automorphism $T: \mathcal{A} \longrightarrow \mathcal{A}$ and an endofunctor $J: \mathcal{A} \longrightarrow \mathcal{A}$ such that

1. $J(M)$ is $\mathcal{E}$-projective, for any $M \in \mathcal{A}$.

2. There are natural transformations $\alpha: i d_{\mathcal{A}} \longrightarrow J$ and $\beta: J \longrightarrow T$ such that, for each $M \in \mathcal{A}$ the pair

$$
\xi_{M} \quad: \quad M \stackrel{\alpha_{M}}{\longrightarrow} J(M) \stackrel{\beta_{M}}{\longrightarrow} T(M),
$$

is an exact pair in $\mathcal{E}$.

Remark 7.7. In the following lemma, recall that we have already defined, for each graded $S$-module $M$ its shifting $M[1]$. Thus $M[1]=\bigoplus_{i \in \mathbb{Z}} M[1]_{i}$, with $M[1]_{i}=M_{i+1}$, for $i \in \mathbb{Z}$. We use also the canonical morphism $\sigma_{M}: M \longrightarrow M[1]$ of degree -1 determined by the identity map on $M$. The isomorphism $\underline{\sigma}_{M}:=$ $L\left(\sigma_{M}\right) \in \operatorname{Hom}_{\mathrm{GMod}-\mathcal{B}}^{-1}(M, M[1])$ plays an important role in the following.

Lemma 7.8. Let $\mathcal{B}$ be a triangular differential $S$-bocs. Then, we have:

1. For each $M \in$ GMod- $\mathcal{B}$, make $T(M)=M[1]$ and, for any morphism $f: M \longrightarrow N$ in GMod-B, make

$$
T(f)=f[1]=\underline{\sigma}_{N} * f * \underline{\sigma}_{M}^{-1}: M[1] \longrightarrow N[1] \text { in GMod- } \mathcal{B} .
$$

Then, we have a degree preserving autofunctor $T:$ GMod- $\mathcal{B} \longrightarrow$ GMod- $\mathcal{B}$ with inverse $T^{-1}$ given by $T^{-1}(M)=M[-1]$ and

$$
T^{-1}(f)=f[-1]=\underline{\sigma}_{N[-1]}^{-1} * f * \underline{\sigma}_{M[-1]}: M[-1] \longrightarrow N[-1] \text { in GMod- } \mathcal{B} .
$$

It is called the shifting autofunctor of GMod- $\mathcal{B}$.

2. For each $(M, u) \in$ TGMod- $\mathcal{B}$, make $T(M, u)=(M[1],-u[1])$ and, for any morphism $f:(M, u) \longrightarrow(N, v)$ in TGMod- $\mathcal{B}$, make

$T(f)=f[1]=\underline{\sigma}_{N} * f * \underline{\sigma}_{M}^{-1}:(M[1],-u[1]) \longrightarrow(N[1],-v[1])$ in TGMod- $\mathcal{B}$.

Then, we have a degree preserving autofunctor $T:$ TGMod- $\mathcal{B} \longrightarrow$ TGMod- $\mathcal{B}$ with inverse $T^{-1}$ given by $T^{-1}(M, u)=(M[-1],-u[-1])$ and

$T^{-1}(f)=f[-1]=\underline{\sigma}_{N[-1]}^{-1} * f * \underline{\sigma}_{M[-1]}:(M[-1],-u[-1]) \longrightarrow(N[-1],-v[-1])$.

The functor $T$ is called the shifting autofunctor of TGMod- $\mathcal{B}$.

3. Furthermore, the functor $T:\left(\operatorname{TGMod}^{0}-\mathcal{B}, \mathcal{E}\right) \longrightarrow\left(\operatorname{TGMod}^{0}-\mathcal{B}, \mathcal{E}\right)$ is an automorphism of exact categories, that is

$$
T(M) \stackrel{T(f)}{\longrightarrow} T(E) \stackrel{T(g)}{\longrightarrow} T(N) \in \mathcal{E} \quad \text { if and only if } M \stackrel{f}{\longrightarrow} E \stackrel{g}{\longrightarrow} N \in \mathcal{E} .
$$

As a consequence, the functors $T$ and $T^{-1}$ preserve the clases of $\mathcal{E}$-injectives and $\mathcal{E}$-projectives. 
Proof. The proof of (1) is easy. In order to prove (2), notice that given $h$ : $M \longrightarrow N$ in GMod- $\mathcal{B}$, using that $\widehat{\delta}\left(\underline{\sigma}_{M}\right)=0$ and Leibniz rule, we have

$$
\widehat{\delta}(h[1])=\widehat{\delta}\left[\underline{\sigma}_{N} * h * \underline{\sigma}_{M}^{-1}\right]=-\underline{\sigma}_{N} * \widehat{\delta}(h) * \underline{\sigma}_{M}^{-1}=-\widehat{\delta}(h)[1] .
$$

Then, given $(M, u) \in$ TGMod- $\mathcal{B}$ we have $\widehat{\delta}(u)+u * u=0$. Thus,

$$
\widehat{\delta}(-u[1])+(-u[1]) *(-u[1])=\widehat{\delta}(u)[1]+u[1] * u[1]=(\widehat{\delta}(u)+u * u)[1]=0,
$$

and $(M[1],-u[1]) \in$ TGMod- $\mathcal{B}$. Moreover, given a morphism $f:(M, u) \longrightarrow(N, v)$ in TGMod- $\mathcal{B}$ we have

$$
\begin{aligned}
\widehat{\delta}(f[1])+(-v[1]) * f[1]-f[1] *(-u[1]) & =-\widehat{\delta}(f)[1]-v[1] * f[1]+f[1] * u[1] \\
& =-(\widehat{\delta}(f)+v * f-f * u)[1]=0,
\end{aligned}
$$

so $f[1]:(M[1],-u[1]) \longrightarrow(N[1],-v[1])$ is a morphism in TGMod- $\mathcal{B}$. Now, it is clear that $T$ is an endofunctor of TGMod- $\mathcal{B}$. It is easy to see that $T$ is an autofunctor with inverse functor $T^{-1}$.

(3): Consider a composable pair $(M, u) \stackrel{f}{\longrightarrow}(E, v) \stackrel{g}{\longrightarrow}(N, w)$ in the category TGMod $^{0}-\mathcal{B}$ and its image

$$
(M[1],-u[1]) \stackrel{f[1]}{\longrightarrow}(E[1],-v[1]) \stackrel{g[1]}{\longrightarrow}(N[1],-w[1])
$$

under the functor $T$. Clearly $g * f=0$ iff $g[1] * f[1]=0$. Notice that for any morphism $h: M \longrightarrow N$ in GMod-S, the first component $L(h)^{0}$ of $L(h)$ is precisely $h$. So, $\underline{\sigma}_{M}^{0}=\sigma_{M}$, for any $M \in$ GMod-S. Hence, we have $(f[1])^{0}=$ $\left(\underline{\sigma}_{E} * f * \underline{\sigma}_{M}^{-1}\right)^{0}=\underline{\sigma}_{E}^{0} f^{0}\left(\underline{\sigma}_{M}^{0}\right)^{-1}=\sigma_{E} f^{0} \sigma_{M}^{-1}$ and similarly for $g$. So we have the commutative diagram in GMod- $S$

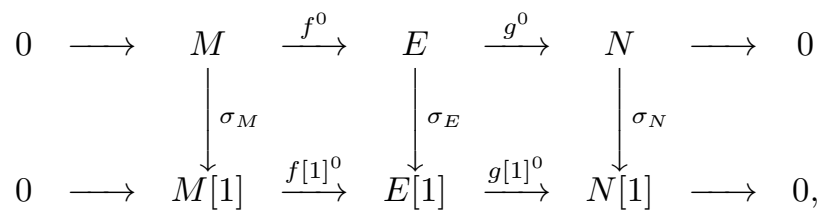

where the vertical morphisms are isomorphisms. Hence, the first row is exact iff the second one is so.

Proposition 7.9. For $\left(M, u_{M}\right) \in$ TGMod- $\mathcal{B}$, we write $u_{M[1]}:=-u_{M}[1]$, so we have $T\left(M, u_{M}\right)=\left(M[1], u_{M[1]}\right)$. There is a degree preserving endofunctor

$$
J: \text { TGMod- } \mathcal{B} \longrightarrow \text { TGMod- } \mathcal{B}
$$

such that, for any $\left(M, u_{M}\right) \in$ TGMod- $\mathcal{B}$ we have

$$
J\left(M, u_{M}\right)=\left(M \oplus M[1],\left(\begin{array}{cc}
u_{M} & \underline{\sigma}_{M}^{-1} \\
0 & u_{M[1]}
\end{array}\right)\right)
$$

and, given a morphism $f:\left(M, u_{M}\right) \longrightarrow\left(N, u_{N}\right)$ in TGMod- $\mathcal{B}$, the morphism $J(f)$ is given by the matrix

$$
J(f)=\left(\begin{array}{cc}
f & 0 \\
0 & f[1]
\end{array}\right): J\left(M, u_{M}\right) \longrightarrow J\left(N, u_{N}\right) .
$$


Proof. Given $(M, u) \in$ TGMod- $\mathcal{B}$, we have

$$
\begin{gathered}
\widehat{\delta}\left(\begin{array}{cc}
u_{M} & \underline{\sigma}_{M}^{-1} \\
0 & u_{M[1]}
\end{array}\right)+\left(\begin{array}{cc}
u_{M} & \underline{\sigma}_{M}^{-1} \\
0 & u_{M[1]}
\end{array}\right) *\left(\begin{array}{cc}
u_{M} & \underline{\sigma}_{M}^{-1} \\
0 & u_{M[1]}
\end{array}\right) \\
=\left(\begin{array}{cc}
\widehat{\delta}\left(u_{M}\right)+u_{M} * u_{M} & u_{M} * \underline{\sigma}_{M}^{-1}+\underline{\sigma}_{M}^{-1} * u_{M[1]} \\
0 & \widehat{\delta}\left(u_{M[1]}\right)+u_{M[1]} * u_{M[1]}
\end{array}\right)=\left(\begin{array}{cc}
0 & 0 \\
0 & 0
\end{array}\right) .
\end{gathered}
$$

So, indeed, we have $J(M, u) \in$ TGMod- $\mathcal{B}$.

Given a morphism $f:\left(M, u_{M}\right) \longrightarrow\left(N, u_{N}\right)$ in TGMod- $\mathcal{B}$, we have

$$
\begin{aligned}
& \widehat{\delta}\left(\begin{array}{cc}
f & 0 \\
0 & f[1]
\end{array}\right)+\left(\begin{array}{cc}
u_{N} & \underline{\sigma}_{N}^{-1} \\
0 & u_{N[1]}
\end{array}\right) *\left(\begin{array}{cc}
f & 0 \\
0 & f[1]
\end{array}\right)-\left(\begin{array}{cc}
f & 0 \\
0 & f[1]
\end{array}\right) *\left(\begin{array}{cc}
u_{M} & \underline{\sigma}_{M}^{-1} \\
0 & u_{M[1]}
\end{array}\right) \\
= & \left(\begin{array}{cc}
\widehat{\delta}(f)+u_{N} * f-f * u_{M} & \widehat{\sigma_{N}^{-1} * f[1]-f * \underline{\sigma}_{M}^{-1}} \\
0 & \widehat{\delta}(f[1])+u_{N[1]} * f[1]-f[1] * u_{M[1]}
\end{array}\right)=\left(\begin{array}{ll}
0 & 0 \\
0 & 0
\end{array}\right) .
\end{aligned}
$$

So, indeed, we have that $J(f): J\left(M, u_{M}\right) \longrightarrow J\left(N, u_{N}\right)$ is a morphism in TGMod- $\mathcal{B}$. Since $T:$ GMod- $\mathcal{B} \longrightarrow$ GMod- $\mathcal{B}$ is a $k$-functor, so is $J$.

Proposition 7.10. For $\left(M, u_{M}\right),\left(N, u_{N}\right) \in$ TGMod- $\mathcal{B}$, we have natural isomorphisms

$$
\eta_{1}: \operatorname{Hom}_{\text {TGMod-B }}^{0}\left(\left(M, u_{M}\right), J\left(N, u_{N}\right)\right) \longrightarrow \operatorname{Hom}_{\text {GMod }-\mathcal{B}}^{0}(M, N)
$$

and

$$
\eta_{2}: \operatorname{Hom}_{\text {TGMod-B }}^{0}\left(J\left(M, u_{M}\right),\left(N, u_{N}\right)\right) \longrightarrow \operatorname{Hom}_{\text {GMod-B }}^{0}(M[1], N) .
$$

Any morphism of twisted graded $\mathcal{B}$-modules $f=\left(f_{1}, f_{2}\right)^{t}:\left(M, u_{M}\right) \longrightarrow J\left(N, u_{N}\right)$, with underlying codomain $N \oplus N[1]$ in GMod- $\mathcal{B}$ is mapped on $\eta_{1}(f)=f_{1}$, and any morphism $g=\left(g_{1}, g_{2}\right): J\left(M, u_{M}\right) \longrightarrow\left(N, u_{N}\right)$ of twisted graded $\mathcal{B}$-modules, with undelying domain $M \oplus M[1]$ in GMod- $\mathcal{B}$ is mapped on $\eta_{2}(g)=g_{2}$.

Proof. (1): Let $f:\left(M, u_{M}\right) \longrightarrow J\left(N, u_{N}\right)$ be a homogeneous morphism of twisted graded $\mathcal{B}$-modules with degree 0 . Thus, $f=\left(f_{1}, f_{2}\right)^{t}: M \longrightarrow N \oplus N[1]$ is a morphism in GMod- $\mathcal{B}$ which satisfies the equation

$$
\widehat{\delta}\left(\begin{array}{l}
f_{1} \\
f_{2}
\end{array}\right)+\left(\begin{array}{cc}
u_{N} & \underline{\sigma}_{N}^{-1} \\
0 & u_{N[1]}
\end{array}\right) *\left(\begin{array}{l}
f_{1} \\
f_{2}
\end{array}\right)-\left(\begin{array}{l}
f_{1} \\
f_{2}
\end{array}\right) * u_{M}=\left(\begin{array}{l}
0 \\
0
\end{array}\right) .
$$

Equivalently, it satisfies the equations:

$$
\begin{array}{ll}
\left(a_{1}\right) & 0=\widehat{\delta}\left(f_{1}\right)+u_{N} * f_{1}+\underline{\sigma}_{N}^{-1} * f_{2}-f_{1} * u_{M} \\
\left(b_{1}\right) & 0=\widehat{\delta}\left(f_{2}\right)+u_{N[1]} * f_{2}-f_{2} * u_{M}
\end{array}
$$

From the equality $\left(a_{1}\right)$, we obtain the following expression of $f_{2}$ in terms of $f_{1}$

$$
f_{2}=\underline{\sigma}_{N} * f_{1} * u_{M}-\underline{\sigma}_{N} * \widehat{\delta}\left(f_{1}\right)-\underline{\sigma}_{N} * u_{N} * f_{1} .
$$


Thus, the linear map $\eta_{1}$ is injective.

Let us see that $\eta_{1}$ is surjective. For this, take $f_{1} \in \operatorname{Hom}_{\mathrm{GMod}-\mathcal{B}}^{0}(M, N)$ and define $f_{2}$ by the equality $\left(a_{1}\right)$, so $f:=\left(f_{1}, f_{2}\right)^{t} \in \operatorname{Hom}_{\mathrm{GMod}-\mathcal{B}}^{0}(M, N \oplus N[1])$. Then,

$$
f \in \operatorname{Hom}_{\mathrm{TGMod}-\mathcal{B}}^{0}\left(\left(M, u_{M}\right), J\left(N, u_{N}\right)\right) \text { iff equality }\left(b_{1}\right) \text { holds. }
$$

We have the following

$$
\begin{aligned}
f_{2} * u_{M} & =\underline{\sigma}_{N} * f_{1} *\left(u_{M} * u_{M}\right)-\underline{\sigma}_{N} * \widehat{\delta}\left(f_{1}\right) * u_{M}-\underline{\sigma}_{N} * u_{N} * f_{1} * u_{M} \\
& =-\underline{\sigma}_{N} *\left[f_{1} * \widehat{\delta}\left(u_{M}\right)+\widehat{\delta}\left(f_{1}\right) * u_{M}+u_{N} * f_{1} * u_{M}\right] \\
u_{N[1]} * f_{2} & =u_{N[1]} * \underline{\sigma}_{N} * f_{1} * u_{M}-u_{N[1]} * \underline{\sigma}_{N} * \widehat{\delta}\left(f_{1}\right)-u_{N[1]} * \underline{\sigma}_{N} * u_{N} * f_{1} \\
& =-\underline{\sigma}_{N} * u_{N} * f_{1} * u_{M}+\underline{\sigma}_{N} * u_{N} * \widehat{\delta}\left(f_{1}\right)+\underline{\sigma}_{N} * u_{N} * u_{N} * f_{1} \\
& =\underline{\sigma}_{N} *\left[-u_{N} * f_{1} * u_{M}+u_{N} * \widehat{\delta}\left(f_{1}\right)-\widehat{\delta}\left(u_{N}\right) * f_{1}\right]
\end{aligned}
$$

and

$$
\begin{aligned}
\widehat{\delta}\left(f_{2}\right) & =-\underline{\sigma}_{N} * \widehat{\delta}\left(f_{1}\right) * u_{M}-\underline{\sigma}_{N} * f_{1} * \widehat{\delta}\left(u_{M}\right)+\underline{\sigma}_{N} * \widehat{\delta}\left(u_{N}\right) * f_{1}-\underline{\sigma}_{N} * u_{N} * \widehat{\delta}\left(f_{1}\right) \\
& =\underline{\sigma}_{N} *\left[-\widehat{\delta}\left(f_{1}\right) * u_{M}-f_{1} * \widehat{\delta}\left(u_{M}\right)+\widehat{\delta}\left(u_{N}\right) * f_{1}-u_{N} * \widehat{\delta}\left(f_{1}\right)\right] .
\end{aligned}
$$

Then, we have that equation $\left(b_{1}\right)$ holds and $\eta_{1}(f)=f_{1}$. So $\eta_{1}$ is an isomorphism, which is clearly a natural transformation.

(2) Let $g: J\left(M, u_{M}\right) \longrightarrow\left(N, u_{N}\right)$ be a homogeneous morphism of twisted graded $\mathcal{B}$-modules with degree 0 . Thus, $g=\left(g_{1}, g_{2}\right): M \oplus M[1] \longrightarrow N$ is a morphism in GMod- $\mathcal{B}$ which satisfies the equation

$$
\widehat{\delta}\left(\begin{array}{ll}
g_{1} & g_{2}
\end{array}\right)+u_{N} *\left(\begin{array}{ll}
g_{1} & g_{2}
\end{array}\right)-\left(\begin{array}{ll}
g_{1} & g_{2}
\end{array}\right) *\left(\begin{array}{cc}
u_{M} & \underline{\sigma}_{M}^{-1} \\
0 & u_{M[1]}
\end{array}\right)=\left(\begin{array}{ll}
0 & 0
\end{array}\right) .
$$

Equivalently, it satisfies the equations:

$$
\begin{array}{ll}
\left(a_{2}\right) & 0=\widehat{\delta}\left(g_{1}\right)+u_{N} * g_{1}-g_{1} * u_{M} \\
\left(b_{2}\right) & 0=\widehat{\delta}\left(g_{2}\right)+u_{N} * g_{2}-g_{1} * \underline{\sigma}_{M}^{-1}-g_{2} * u_{M[1]}
\end{array}
$$

From the equality $\left(b_{2}\right)$, we obtain the following expression of $g_{1}$ in terms of $g_{2}$

$$
g_{1}=\widehat{\delta}\left(g_{2}\right) * \underline{\sigma}_{M}+u_{N} * g_{2} * \underline{\sigma}_{M}-g_{2} * u_{M[1]} * \underline{\sigma}_{M} .
$$

Thus, the linear map $\eta_{2}$ is injective.

Let us see that $\eta_{2}$ is surjective. For this, take $g_{2} \in \operatorname{Hom}_{\text {GMod- } \mathcal{B}}^{0}(M[1], N)$ and define $g_{1}$ by the equality $\left(b_{2}\right)$, so $g:=\left(g_{1}, g_{2}\right) \in \operatorname{Hom}_{\text {GMod- } \mathcal{B}}^{0}(M \oplus M[1], N)$. Then,

$$
g \in \operatorname{Hom}_{\text {TGMod-B }}^{0}\left(J\left(M, u_{M}\right),\left(N, u_{N}\right)\right) \text { iff equality }\left(a_{2}\right) \text { holds. }
$$

We have the equalities

$$
\begin{aligned}
g_{1} * u_{M} & =\widehat{\delta}\left(g_{2}\right) * \underline{\sigma}_{M} * u_{M}+u_{N} * g_{2} * \underline{\sigma}_{M} * u_{M}-g_{2} * u_{M[1]} * \underline{\sigma}_{M} * u_{M} \\
& =-\widehat{\delta}\left(g_{2}\right) * u_{M[1]} * \underline{\sigma}_{M}-u_{N} * g_{2} * u_{M[1]} * \underline{\sigma}_{M}+g_{2} * u_{M[1]} * u_{M[1]} * \underline{\sigma}_{M} \\
& =-\left[\widehat{\delta}\left(g_{2}\right) * u_{M[1]}+u_{N} * g_{2} * u_{M[1]}+g_{2} *\left(u_{M[1]}\right)\right] * \underline{\sigma}_{M}
\end{aligned}
$$




$$
\begin{aligned}
u_{N} * g_{1} & =u_{N} * \widehat{\delta}\left(g_{2}\right) * \underline{\sigma}_{M}+u_{N} * u_{N} * g_{2} * \underline{\sigma}_{M}-u_{N} * g_{2} * u_{M[1]} * \underline{\sigma}_{M} \\
& =\left[u_{N} * \widehat{\delta}\left(g_{2}\right)-\widehat{\delta}\left(u_{N}\right) * g_{2}-u_{N} * g_{2} * u_{M[1]}\right] * \underline{\sigma}_{M}
\end{aligned}
$$

and

$$
\begin{aligned}
\widehat{\delta}\left(g_{1}\right) & =\widehat{\delta}\left(u_{N}\right) * g_{2} * \underline{\sigma}_{M}-u_{N} * \widehat{\delta}\left(g_{2}\right) * \underline{\sigma}_{M}-\widehat{\delta}\left(g_{2}\right) * u_{M[1]} * \underline{\sigma}_{M}-g_{2} * \widehat{\delta}\left(u_{M[1]}\right) * \underline{\sigma}_{M} \\
& =\left[\widehat{\delta}\left(u_{N}\right) * g_{2}-u_{N} * \widehat{\delta}\left(g_{2}\right)-\widehat{\delta}\left(g_{2}\right) * u_{M[1]}-g_{2} * \widehat{\delta}\left(u_{M[1]}\right)\right] * \underline{\sigma}_{M} .
\end{aligned}
$$

Then, we have that equation $\left(a_{2}\right)$ holds and $\eta_{2}(g)=g_{2}$. So $\eta_{2}$ is an isomorphism, which is clearly a natural transformation.

Corollary 7.11. For each $(M, u) \in$ TGMod- $\mathcal{B}$, the twisted graded $\mathcal{B}$-module $J(M, u)$ is $\mathcal{E}$-projective and $\mathcal{E}$-injective in the exact category $\left(\operatorname{TGMod}^{0}-\mathcal{B}, \mathcal{E}\right)$.

Proof. Let us write $\underline{M}=\left(M, u_{M}\right)$ for the objects of TGMod- $\mathcal{B}$. For each exact pair $\underline{M} \stackrel{f}{\longrightarrow} \underline{E} \stackrel{g}{\longrightarrow} \underline{N}$ in $\mathcal{E}$, we have a commutative diagram

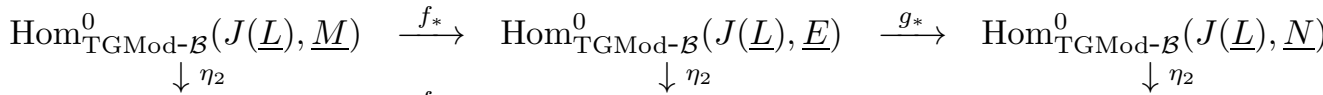

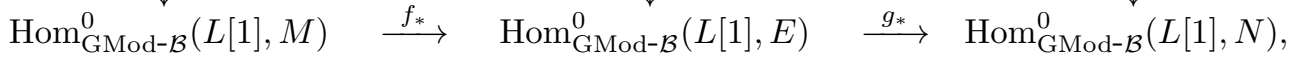

where the second row is exact with $g_{*}$ surjective and $f_{*}$ injective. Since $\eta_{2}$ is a natural isomorphism, the first row is exact with $g_{*}$ surjective and $f_{*}$ injective. So $J(\underline{L})$ is $\mathcal{E}$-projective.

The argument showing that $J(\underline{L})$ is $\mathcal{E}$-injective is similar, now using $\eta_{1}$.

For the rest of this section, we consider the restriction functors

$$
T: \operatorname{TGMod}^{0}-\mathcal{B} \longrightarrow \mathrm{TGMod}^{0}-\mathcal{B} \text { and } J: \mathrm{TGMod}^{0}-\mathcal{B} \longrightarrow \mathrm{TGMod}^{0}-\mathcal{B}
$$

denoted with the same symbols used for the functors $T$ and $J$ considered before.

Proposition 7.12. There are natural transformations $\alpha: i d_{\mathrm{TGMod}^{0} \mathcal{B}} \longrightarrow J$ and $\beta: J \longrightarrow T$ such that for each $\underline{M} \in$ TGMod- $\mathcal{B}$ we have an $\mathcal{E}$-conflation

$$
\underline{M} \stackrel{\alpha_{\underline{M}}}{\longrightarrow} J(\underline{M}) \stackrel{\beta_{\underline{N}}}{\longrightarrow} T(\underline{M}) .
$$

For $\underline{M} \in \mathrm{GM}$ od- $\mathcal{B}$, these morphisms are given by

$$
\alpha_{\underline{M}}=\left(\mathbb{I}_{M}, 0\right)^{t}: \underline{M} \longrightarrow J(\underline{M}) \text { and } \beta_{\underline{M}}=\left(0, \mathbb{I}_{M[1]}\right): J(\underline{M}) \longrightarrow \underline{N} .
$$

Proof. Consider the 0-degree homogeneous morphisms of twisted $\mathcal{B}$-modules

$$
\alpha_{\underline{M}}:=\eta_{1}^{-1}\left(\mathbb{I}_{M}\right) \text { and } \beta_{\underline{M}}:=\eta_{2}^{-1}\left(\mathbb{I}_{M[1]}\right),
$$

which are described explicitely by the formulas for the inverses of $\eta_{1}$ and $\eta_{2}$ given in the proof of (7.10).

From (7.11) and (7.12), we immediately obtain the following. 
Corollary 7.13. The $\mathcal{E}$-projective and the $\mathcal{E}$-injective objects in the exact category $\left(\operatorname{TGMod}^{0}-\mathcal{B}, \mathcal{E}\right)$ are the direct summands of the objects $J(\underline{M})$, for $\underline{M} \in$ TGMod- $\mathcal{B}$.

Proposition 7.14. Let $f: \underline{M} \longrightarrow \underline{N}$ be a morphism in $\mathrm{TGMod}^{0}-\mathcal{B}$. Then, $f$ is homotopically trivial iff it factors through an $\mathcal{E}$-projective twisted $\mathcal{B}$-module.

Proof. Since the $\mathcal{E}$-projective twisted $\mathcal{B}$-modules coincide with the $\mathcal{E}$-injective twisted $\mathcal{B}$-modules, a morphism $f: \underline{M} \longrightarrow \underline{N}$ factors through an $\mathcal{E}$-projective iff it factors through $\alpha_{\underline{M}}$. So we have to show that $f$ factors through $\alpha_{\underline{M}}$ iff it is homotopically trivial.

Suppose first that $f$ factors through $\alpha_{\underline{M}}$ Then there is $h=\left(h_{1}, h_{2}\right) \in$ $\operatorname{Hom}_{\text {TGMod-B }}^{0}(J(\underline{M}), \underline{N})$ such that $f=h * \underline{\alpha}_{\underline{M}}$. Then, $f=h_{1}$ and the first component of $h$ is given by

$$
h_{1}=\widehat{\delta}\left(h_{2}\right) * \underline{\sigma}_{M}+u_{N} * h_{2} * \underline{\sigma}_{M}-h_{2} * u_{M[1]} * \underline{\sigma}_{M} .
$$

Hence,

$$
f=\widehat{\delta}\left(h_{2} * \underline{\sigma}_{M}\right)+u_{N} *\left(h_{2} * \underline{\sigma}_{M}\right)+\left(h_{2} * \underline{\sigma}_{M}\right) * u_{M},
$$

with $h_{2} * \underline{\sigma}_{M} \in \operatorname{Hom}_{\text {GMod- } \mathcal{B}}^{-1}(M, N)$. So, $f$ is homotopically trivial.

Assume now that $f: \underline{M} \longrightarrow \underline{N}$ is homotopically trivial and take a morphism $g \in \operatorname{Hom}_{\text {GMod-B }}^{-1}(M, N)$ such that $f=\widehat{\delta}(g)+u_{N} * g+g * u_{M}$. Now, consider the morphism $h_{2}:=g * \underline{\sigma}_{M}^{-1} \in \operatorname{Hom}_{\mathrm{GMod}-\mathcal{B}}^{0}(M[1], N)$. Then, we have

$$
\begin{aligned}
f & =\widehat{\delta}\left(h_{2} * \underline{\sigma}_{M}\right)+u_{N} *\left(h_{2} * \underline{\sigma}_{M}\right)+\left(h_{2} * \underline{\sigma}_{M}\right) * u_{M} \\
& =\widehat{\delta}\left(h_{2}\right) * \underline{\sigma}_{M}+u_{N} * h_{2} * \underline{\sigma}_{M}-h_{2} * u_{M[1]} * \underline{\sigma}_{M} .
\end{aligned}
$$

Then, $h=\left(f, h_{2}\right) \in \operatorname{Hom}_{\text {TGMod-B }}^{0}(J(\underline{M}), \underline{N})$ satisfies $f=h * \alpha_{\underline{M}}$.

Definition 7.15. Let $\mathcal{B}$ be a triangular differential graded $S$-bocs. Then, a sequence of morphisms

$$
\left(M, u_{M}\right) \stackrel{\underline{f}}{\longrightarrow}\left(E, u_{E}\right) \stackrel{\underline{g}}{\longrightarrow}\left(N, u_{N}\right) \stackrel{\underline{h}}{\longrightarrow} T\left(M, u_{M}\right)
$$

in the homotopic category $\underline{\mathrm{TGMod}}^{0}-\mathcal{B}$ such that

$$
\xi:\left(M, u_{M}\right) \stackrel{f}{\longrightarrow}\left(E, u_{E}\right) \stackrel{g}{\longrightarrow}\left(N, u_{N}\right)
$$

is an exact pair in $\mathcal{E}$ and we have a commutative diagram of the form

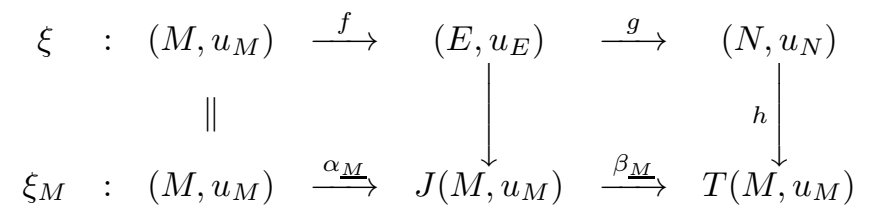

in $\mathrm{TGMod}^{0}-\mathcal{B}$ is called a canonical triangle in $\underline{\mathrm{TGMod}}^{0}-\mathcal{B}$. Notice that, in this case, we have $\xi_{M} h=\xi$. Now, consider the class $\mathcal{T}$ of sequences of morphisms

$$
X \stackrel{u}{\longrightarrow} Y \stackrel{v}{\longrightarrow} Z \stackrel{w}{\longrightarrow} T X
$$


in $\underline{\mathrm{TGMod}}^{0}-\mathcal{B}$ which are isomorphic to canonical triangles. That is, there is a canonical triangle $M \stackrel{\underline{f}}{\longrightarrow} E \stackrel{\underline{g}}{\longrightarrow} N \stackrel{\underline{h}}{\longrightarrow} T M$ and isomorphisms $a, b, c$ such that the following diagram commutes

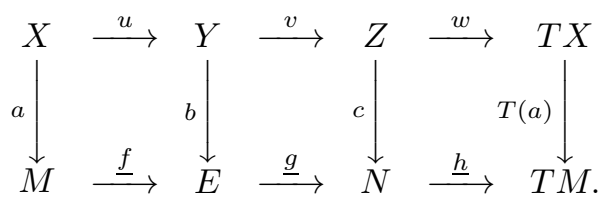

The elements of $\mathcal{T}$ are called the triangles of ${\underline{\mathrm{TGMod}^{0}}}^{0} \mathcal{B}$.

The following result follows from the general theory for special Frobenius categories, see [5] and [1].

Theorem 7.16. Let $\mathcal{B}$ be a triangular differential graded $S$-bocs. Then the category TGMod $^{0}-\mathcal{B}$ is a special Frobenius category with the automorphism $T$ of (7.8) and the endofunctor $J$ described in (7.9), both restricted to $\mathrm{TGMod}^{0}-\mathcal{B}$.

The stable category $\underline{\mathrm{TGMod}}^{0}-\mathcal{B}$ with the automorphism $T$ and the class of triangles $\mathcal{T}$ defined above is a triangulated category.

\section{Quasi-isomorphisms of twisted modules}

Before, the proof of our theorem (8.3), we fix some useful notation.

Remark 8.1. Given a graded differential $S$-bocs $\mathcal{B}=(C, \mu, \epsilon, \delta)$, the equivalence of categories $F:$ GMod- $\mathcal{B} \longrightarrow$ GMod- $\mathcal{B}$ described in (4.4) permits to transfer the differential $\widehat{\delta}$ of the category GMod- $\mathcal{B}$ to a differential $\widehat{\delta}$ on the graded category GModI- $\mathcal{B}$.

Thus, given a homogeneous morphism $f=\left(f^{0}, f^{1}\right): M \longrightarrow N$ in GModI- $\mathcal{B}$, we have $\widehat{\delta}(f)=\left(0, \widehat{\delta}(f)^{1}\right)$, where for any homogeneous elements $m \in M$ and $c \in \bar{C}$, we have

$$
\widehat{\delta}(f)^{1}(c)[m]=(-1)^{|f|+|m|+1} f^{1}(\delta(c))[m] .
$$

If $g: M \longrightarrow N$ is a morphism in GMod- $\mathcal{B}$, then $\widehat{\delta}(F(g))=F(\widehat{\delta}(g))$. So $F$ determines an equivalence of the differential graded categories GMod- $\mathcal{B}$ and GModI- $\mathcal{B}$.

Then, the category of twisted $\mathcal{B}$-modules TGModI- $\mathcal{B}$ is formed by the pairs $\underline{M}=\left(M, u_{M}\right)$, with $u_{M} \in \operatorname{Hom}_{\mathrm{GModI}-\mathcal{B}}^{1}(M, N)$ such that $\widehat{\delta}\left(u_{M}\right)+u_{M} * u_{M}=0$.

A homogeneous morphism $f: \underline{M} \longrightarrow \underline{N}$ of degree $d$ in TGModI- $\mathcal{B}$ is a homogeneous morphism $f: M \longrightarrow N$ of degree $d$ in GModI- $\mathcal{B}$ such that the equality $\widehat{\delta}(f)+u_{N} * f-(-1)^{d} f * u_{M}=0$ holds.

A homogeneous morphism $f: \underline{M} \longrightarrow \underline{N}$ in TGModI ${ }^{0}-\mathcal{B}$ is called homotopically trivial iff there is a homogeneous morphism $h: M \longrightarrow N$ in GModI- $\mathcal{B}$ of degree -1 such that $f=\widehat{\delta}(h)+u_{N} * h+h * u_{M}$.

For the sake of notational simplicity, from now on, given $f=\left(f^{0}, f^{1}\right)$ : $M \longrightarrow N$ and $g=\left(g^{0}, g^{1}\right): N \longrightarrow L$ in GModI- $\mathcal{B}$, we write

$$
g^{0} f^{1}:=\left[\bar{C} \stackrel{f^{1}}{\longrightarrow} \operatorname{Hom}_{\mathrm{GMod}-k}(M, N) \stackrel{g_{*}^{0}}{\longrightarrow} \operatorname{Hom}_{\mathrm{GMod}-k}(M, L)\right],
$$




$$
g^{1} f^{0}:=\left[\bar{C} \stackrel{g^{1}}{\longrightarrow} \operatorname{Hom}_{\mathrm{GMod}-k}(N, L) \stackrel{f_{*}^{0}}{\longrightarrow} \operatorname{Hom}_{\mathrm{GMod}-k}(M, L)\right],
$$

and $g^{1} \cdot f^{1}:=(g * f)^{1}-g^{0} f^{1}-g^{1} f^{0}: \bar{C} \longrightarrow \operatorname{Hom}_{\mathrm{GMod}-k}(M, L)$. Thus, the maps $g^{0} f^{1}, g^{1} f^{0}$ and $g^{1} \cdot f^{1}$ are morphisms of graded $S$-S-bimodules. Then, as mentioned in (6.4), there is a formula to compute $g^{1} \cdot f^{1}$. Namely, given $c \in \bar{C}$ with $\bar{\mu}(c)=\sum_{s} c_{s}^{1} \otimes c_{s}^{2}$, with $c_{s}^{1}, c_{s}^{2} \in \bar{C}$, we have

$$
\left(g^{1} \cdot f^{1}\right)(c)=\sum_{s} g^{1}\left(c_{s}^{2}\right) f^{1}\left(c_{s}^{1}\right)
$$

Therefore, $(g * f)^{0}=g^{0} f^{0}$ and $(g * f)^{1}=g^{0} f^{1}+g^{1} f^{0}+g^{1} \cdot f^{1}$.

Then, the equality $\widehat{\delta}\left(u_{M}\right)+u_{M} * u_{M}=0$ is equivalent to

$$
(A): \quad\left\{\begin{array}{l}
0=\left(u_{M}^{0}\right)^{2} \\
0=\widehat{\delta}\left(u_{M}\right)^{1}+u_{M}^{0} u_{M}^{1}+u_{M}^{1} u_{M}^{0}+u_{M}^{1} \cdot u_{M}^{1} .
\end{array}\right.
$$

A homogeneous morphism $f: \underline{M} \longrightarrow \underline{N}$ of degree 0 in TGModI- $\mathcal{B}$ is a morphism $f=\left(f^{0}, f^{1}\right): M \longrightarrow N$ of degree 0 in GModI- $\mathcal{B}$ such that

$$
\left\{\begin{array}{l}
0=u_{N}^{0} f^{0}-f^{0} u_{M}^{0} \\
0=\widehat{\delta}(f)^{1}+u_{N}^{0} f^{1}+u_{N}^{1} f^{0}+u_{N}^{1} \cdot f^{1}-f^{0} u_{M}^{1}-f^{1} u_{M}^{0}-f^{1} \cdot u_{M}^{1} .
\end{array}\right.
$$

In particular, $f^{0}:\left(M, u_{M}^{0}\right) \longrightarrow\left(N, u_{N}^{0}\right)$ is a morphism of complexes of right $S$-modules.

For the proof of the following result, it is convenient to extend the preceding notations as follows.

Remark 8.2. Let $\mathcal{B}=(C, \mu, \epsilon, \delta)$ be a triangular differential graded $S$-bocs and consider its triangular filtration $0=\bar{C}_{0} \subseteq \bar{C}_{1} \subseteq \cdots \subseteq \bar{C}_{i} \subseteq \bar{C}_{i+1} \subseteq \cdots$

For any $M, N, L \in$ GMod- $S, f_{i}^{1} \in \operatorname{Hom}_{\text {GMod- } S}\left(M \otimes_{S} \bar{C}_{i}, N\right)$, and $g_{i}^{1} \in$ $\operatorname{Hom}_{\text {GMod- } S}\left(N \otimes_{S} \bar{C}_{i}, L\right)$ we can consider the morphism of graded right $S$ modules $g_{i}^{1} * f_{i}^{1} \in \operatorname{Hom}_{\mathrm{GMod}-S}\left(M \otimes_{S} \bar{C}_{i+1}, L\right)$ defined as the composition

$$
M \otimes_{S} \bar{C}_{i+1} \stackrel{i d_{M} \otimes \bar{\mu}}{\longrightarrow} M \otimes_{S} \bar{C}_{i} \otimes_{S} \bar{C}_{i} \stackrel{f_{i}^{1} \otimes i d_{\bar{C}_{i}}}{\longrightarrow} N \otimes_{S} \bar{C}_{i} \stackrel{g_{i}^{1}}{\longrightarrow} L .
$$

For each $i \geq 0$, we have the canonical isomorphism

$$
\eta_{i}: \operatorname{Hom}_{\mathrm{GMod}-S}\left(M \otimes_{S} \bar{C}_{i}, L\right) \longrightarrow \operatorname{Hom}_{\mathrm{GMod}-S-S}\left(\bar{C}_{i}, \operatorname{Hom}_{\mathrm{GMod}-k}(M, L)\right) .
$$

We can define the product morphism: $\eta_{i}\left(g_{i}^{1}\right) \cdot \eta_{i}\left(f_{i}^{1}\right):=\eta_{i+1}\left(g_{i}^{1} * f_{i}^{1}\right)$.

Then, given any two morphisms $f_{i}^{1} \in \operatorname{Hom}_{\mathrm{GMod}-S-S}\left(\bar{C}_{i}, \operatorname{Hom}_{\mathrm{GMod}-k}(M, N)\right)$ and $g_{i}^{1} \in \operatorname{Hom}_{\text {GMod-S-S }}\left(\bar{C}_{i}, \operatorname{Hom}_{\mathrm{GMod}-k}(N, L)\right)$, we have their product morphism $g_{i}^{1} \cdot f_{i}^{1} \in \operatorname{Hom}_{\mathrm{GMod}-S-S}\left(\bar{C}_{i+1}, \operatorname{Hom}_{\mathrm{GMod}-k}(M, L)\right)$ which is computed on each element $c \in \bar{C}_{i+1}$ as

$$
\left(g_{i}^{1} \cdot f_{i}^{1}\right)(c)=\sum_{j} g_{i}^{1}\left(c_{j}^{2}\right) f_{i}^{1}\left(c_{j}^{1}\right), \text { where } \bar{\mu}(c)=\sum_{j} c_{j}^{1} \otimes c_{j}^{2} \text { with } c_{j}^{1}, c_{j}^{2} \in \bar{C}_{i} .
$$


Given $g^{0} \in \operatorname{Hom}_{\text {GMod- } S}(N, L)$ and $f_{i}^{1} \in \operatorname{Hom}_{\text {GMod- } S-S}\left(\bar{C}_{i}, \operatorname{Hom}_{\text {GMod- } k}(M, N)\right)$, the morphism $g^{0} f_{i}^{1} \in \operatorname{Hom}_{\text {GMod-S-S }}\left(\bar{C}_{i}, \operatorname{Hom}_{k}(M, L)\right)$ is defined by $\left(g^{0} f_{i}^{1}\right)(c)=$ $g^{0} f_{i}^{1}(c)$. Similarly, given morphisms $g_{i}^{1} \in \operatorname{Hom}_{\mathrm{GMod}-S-S}\left(\bar{C}_{i}, \operatorname{Hom}_{\mathrm{GMod}-k}(N, L)\right)$ and $f^{0} \in \operatorname{Hom}_{\mathrm{GMod}-S}(M, N), g_{i}^{1} f^{0} \in \operatorname{Hom}_{\mathrm{GMod}-S-S}\left(\bar{C}_{i}, \operatorname{Hom}_{\mathrm{GMod}-k}(M, L)\right)$ is defined by $\left(g_{i}^{1} f^{0}\right)(c)=g_{i}^{1}(c) f^{0}$.

The following formulas hold:

$$
h_{i}^{1} \cdot g^{0} f_{i}^{1}=h_{i}^{1} g^{0} \cdot f_{i}^{1}, \quad h^{0}\left(f_{i}^{1} \cdot g_{i}^{1}\right)=h^{0} f_{i}^{1} \cdot g_{i}^{1}, \text { and }\left(h_{i}^{1} \cdot g_{i}^{1}\right) f^{0}=h_{i}^{1} \cdot g_{i}^{1} f^{0} .
$$

If the morphism $h_{i+1}^{1}$ restricts to $h_{i}^{1}$ and the morphism $f_{i+1}^{1}$ restricts to $f_{i}^{1}$, from the coassociativity of the comultiplication $\bar{\mu}$, we have the associativity formula

$$
h_{i+1}^{1} \cdot\left(g_{i}^{1} \cdot f_{i}^{1}\right)=\left(h_{i}^{1} \cdot g_{i}^{1}\right) \cdot f_{i+1}^{1} .
$$

Since the bocs $\mathcal{B}$ is triangular, we have $\delta\left(\bar{C}_{i+1}\right) \subseteq Z\left(\bar{C}_{i+1}\right)$. Then, for a homogeneous element $\widehat{f}_{i}^{1} \in \operatorname{Hom}_{\mathrm{GMod}-S-S}\left(\bar{C}_{i}+Z\left(\bar{C}_{i+1}\right), \operatorname{Hom}_{\mathrm{GMod}-k}(M, N)\right)$, we can define the morphism $\widehat{d}\left(\widehat{f}_{i}^{1}\right) \in \operatorname{Hom}_{\mathrm{GMod}-S-S}\left(\bar{C}_{i+1}, \operatorname{Hom}_{\mathrm{GMod}-k}(M, N)\right)$ by

$$
\widehat{d}\left(\widehat{f}_{i}^{1}\right)(c)[m]=(-1)^{|m|+\left|\widehat{f}_{i}^{1}\right|+1} \widehat{f}_{i}^{1}(\delta(c))[m],
$$

for any $c \in \bar{C}_{i+1}$ and any homogeneous element $m \in M$.

Given a homogeneous $\widehat{g}_{i}^{1} \in \operatorname{Hom}_{\mathrm{GMod}-S-S}\left(\bar{C}_{i}+Z\left(\bar{C}_{i+1}\right), \operatorname{Hom}_{\mathrm{GMod}-k}(N, L)\right)$, the following Leibniz formula for morphisms $\bar{C}_{i+1} \longrightarrow \operatorname{Hom}_{\text {GMod-k }}(M, L)$ holds

$$
\widehat{d}\left(g_{i}^{1} \cdot f_{i}^{1}\right)=\widehat{d}\left(\widehat{g}_{i}^{1}\right) \cdot f_{i}^{1}+(-1)^{\left|g_{i}^{1}\right|} g_{i}^{1} \cdot \widehat{d}\left(\widehat{f}_{i}^{1}\right),
$$

where $f_{i}^{1}$ and $g_{i}^{1}$ denote the restrictions of $\widehat{f}_{i}^{1}$ and $\widehat{g}_{i}^{1}$ from their common domain $\bar{C}_{i}+Z\left(\bar{C}_{i+1}\right)$ to $\bar{C}_{i}$; thus the morphism $g_{i}^{1} \cdot f_{i}^{1}$ is defined on $\bar{C}_{i+1}$, hence it is defined on $\bar{C}_{i}+Z\left(\bar{C}_{i+1}\right)$; the morphisms $\widehat{d}\left(\widehat{g}_{i}^{1}\right)$ and $\widehat{d}\left(\widehat{f}_{i}^{1}\right)$ are defined on $\bar{C}_{i+1}$, hence on $\bar{C}_{i}$.

Theorem 8.3. Assume that $S$ is a finite product of copies of the field $k$. Let $(M, u) \in$ TGMod-B. Then, the twisted $\mathcal{B}$-module $(M, u)$ is homotopically trivial in $\mathrm{TGModI}^{0}-\mathcal{B}$ iff the complex of right $S$-modules $\left(M, u^{0}\right)$ is acyclic, that is $H^{i}\left(M, u^{0}\right)=0$, for all $i \in \mathbb{Z}$.

Proof. If $(M, u)$ is homotopically trivial, then $\mathbb{I}_{M}=\left(i d_{M}, 0\right)$ is homotopic to the zero map in TGModI ${ }^{0}-\mathcal{B}$. Then, there is a morphism $h \in \operatorname{Hom}_{\mathrm{GModI}-\mathcal{B}}^{-1}(M, M)$ such that $\mathbb{I}_{M}=\widehat{\delta}(h)+u * h+h * u$. Looking at the first components, we obtain $i d_{M}=u^{0} h^{0}+h^{0} u^{0}$. So, $\left(M, u^{0}\right)$ is a homotopically trivial complex of right $S$-modules, which implies that $H^{i}\left(M, u^{0}\right)=0$, for all $i \in \mathbb{Z}$.

Now, assume that $\left(M, u^{0}\right)$ is an acyclic complex of right $S$-modules. Then, for instance from [4] $(0.3)$, we know that $\left(M, u^{0}\right)$ is homotopically trivial. So, there is a homogeneous morphism $h^{0}: M \longrightarrow M$ of right $S$-modules of degree -1 such that the following equality holds

$$
\left(\Delta^{0}\right): \quad i d_{M}=u^{0} h^{0}+h^{0} u^{0} .
$$


We want to show that $(M, u)$ is homotopically trivial, so we are looking for a homogeneous morphism of $S$-S-bimodules $h^{1}: \bar{C} \longrightarrow \operatorname{Hom}_{\mathrm{GMod}-k}(M, M)$ of degree -1 such that $h=\left(h^{0}, h^{1}\right)$ satisfies $\widehat{\delta}(h)+u * h+h * u=\mathbb{I}_{M}$. That is such that the following equality holds

$$
\Delta^{1}:=\widehat{\delta}(h)^{1}+u^{0} h^{1}+u^{1} h^{0}+u^{1} \cdot h^{1}+h^{0} u^{1}+h^{1} u^{0}+h^{1} \cdot u^{1}=0 .
$$

Write $E_{M}:=\operatorname{Hom}_{\text {GMod- } k}(M, M)$ and make $h_{0}^{1}=0 \in \operatorname{Hom}_{\text {GMod-S-S }}^{-1}\left(\bar{C}_{0}, E_{M}\right)$. We will construct, for each $i \geq 0$, a morphism $h_{i+1}^{1} \in \operatorname{Hom}_{\mathrm{GMod}-S-S}^{-1}\left(\bar{C}_{i+1}, E_{M}\right)$ such that

$$
\Delta_{i}^{1}:=\widehat{d}\left(h_{i+1}^{1}\right)+u^{0} h_{i+1}^{1}+u_{i+1}^{1} h^{0}+u_{i}^{1} \cdot h_{i}^{1}+h^{0} u_{i+1}^{1}+h_{i+1}^{1} u^{0}+h_{i}^{1} \cdot u_{i}^{1}=0
$$

and $h_{i+1}^{1}$ restricts to $h_{i}^{1}$, for all $i$. Here, $u_{i}^{1}$ denotes the restriction of $u^{1}$ to $\bar{C}_{i}$. Once we evaluate $u_{i}^{1}$, we can skip the subindex: $u_{i}^{1}(c)=u^{1}(c)$, for $c \in \bar{C}_{i}$.

Once we have done this, we can define $h^{1} \in \operatorname{Hom}_{\text {GMod-S-S }}^{-1}\left(\bar{C}, E_{M}\right)$, by $h^{1}(c)=h_{i}^{1}(c)$, whenever $c \in \bar{C}_{i}$. So $\Delta^{1}(c)=\Delta_{i}^{1}(c)=0$, for $c \in \bar{C}_{i+1}$. Hence $\Delta^{1}=0$ and we are done.

We require for this construction a special vector space basis $\mathbb{B}_{i+1}$ of $\bar{C}_{i+1}$ consisting of homogeneous elements. In order to describe this basis, we will consider, for each $i \geq 0$, decompositions of graded $S$-S-bimodules of the form

$$
\bar{C}_{i+1}=\bar{C}_{i} \oplus V_{i+1} \oplus W_{i+1},
$$

where $\bar{C}_{i}+Z\left(\bar{C}_{i+1}\right)=\bar{C}_{i} \oplus V_{i+1}$ and $V_{i+1} \subseteq Z\left(\bar{C}_{i+1}\right)$. By assumption, we have a decomposition $1=\sum_{s=1}^{n} e_{s}$ of the unit element of the algebra $S$ as a sum of central primitive orthogonal idempotents. The special basis we are interested in has the form $\mathbb{B}_{i+1}=\bigcup_{s, t \in[1, n]} \mathbb{B}_{i+1}(s, t)$, where each subset $\mathbb{B}_{i+1}(s, t)$ is the basis of $e_{t} \bar{C}_{i+1} e_{s}$ defined recursively as follows. At the base $i=0$, we have $\mathbb{B}_{1}(s, t):=\mathbb{B}_{1}^{v}(s, t) \cup \mathbb{B}_{1}^{w}(s, t)$, where $\mathbb{B}_{1}^{v}(s, t)$ and $\mathbb{B}_{1}^{w}(s, t)$ are basis formed by homogeneous elements of $e_{t} V_{1} e_{s}$ and $e_{t} W_{1} e_{s}$, respectively. Once we have defined a basis $\mathbb{B}_{i}$ of $\bar{C}_{i}$, for $i \geq 1$, we define

$$
\mathbb{B}_{i+1}(s, t):=\mathbb{B}_{i}(s, t) \cup \mathbb{B}_{i+1}^{v}(s, t) \cup \mathbb{B}_{i+1}^{w}(s, t),
$$

where $\mathbb{B}_{i+1}^{v}(s, t)$ and $\mathbb{B}_{i+1}^{w}(s, t)$ are basis consisting of homogeneous elements of $e_{t} V_{i+1} e_{s}$ and $e_{t} W_{i+1} e_{s}$, respectively

Step 1: The construction of $h_{1}^{1}: \bar{C}_{1} \longrightarrow E_{M}$.

In order to define the homogeneous morphism of $S$-S-bimodules $h_{1}^{1}$ we want, it will be enough to give, for each homogeneous basic element $c \in \mathbb{B}_{1}(s, t)$, a homogeneous element $h_{1}^{1}(c) \in \operatorname{Hom}_{\mathrm{GMod}-k}\left(M e_{t}, M e_{s}\right)$ of degree $|c|-1$ satisfying the equation $\Delta_{0}^{1}(c)=0$.

Start with a basic element $c \in \mathbb{B}_{1}^{v}(s, t)$, so $c \in Z\left(\bar{C}_{1}\right)$. Consider the homogeneous morphism $f_{0}(c): M e_{t} \longrightarrow M e_{s}$ of degree $|c|$ given by $f_{0}(c):=$ $u_{1}^{1}(c) h^{0}+h^{0} u_{1}^{1}(c)$. Since $i d_{M}=u^{0} h^{0}+h^{0} u^{0}$, we have

$$
\begin{aligned}
u^{0} f_{0}(c)-f_{0}(c) u^{0}= & u^{0} u^{1}(c) h^{0}+u^{0} h^{0} u^{1}(c)-u^{1}(c) h^{0} u^{0}-h^{0} u^{1}(c) u^{0} \\
= & u^{0} u^{1}(c) h^{0}+u^{1}(c)-h^{0} u^{0} u^{1}(c) \\
& -u^{1}(c)+u^{1}(c) u^{0} h^{0}-h^{0} u^{1}(c) u^{0} \\
= & {\left[u^{0} u_{1}^{1}(c)+u_{1}^{1}(c) u^{0}\right] h^{0}-h^{0}\left[u^{0} u_{1}^{1}(c)+u_{1}^{1}(c) u^{0}\right] . }
\end{aligned}
$$


Since $\bar{\mu}(c)=0$ and $\bar{\delta}(c)=0$, from (8.1) (A), we get $u^{0} u^{1}(c)+u^{1}(c) u^{0}=0$. Therefore,

$$
u^{0} f_{0}(c)=f_{0}(c) u^{0} .
$$

In the following, given $N \in$ GMod- $k$, we denote by $\sigma^{[i]}: N \longrightarrow N[i]$ the homogeneous morphism of degree $-i$ which acts as the identity on the underlying non-graded spaces.

Consider the morphism $\tau:=\sigma^{[|c|]}: M e_{s} \longrightarrow M e_{s}[|c|]$. Then, the homogeneous morphism $\tau f_{0}(c): M e_{t} \longrightarrow M e_{s}[|c|]$ of degree 0 satisfies

$$
\tau f_{0}(c) u^{0}-\tau u^{0} \tau^{-1} \tau f_{0}(c)=\tau\left(f_{0}(c) u^{0}-u^{0} f_{0}(c)\right)=0 .
$$

Thus $\tau f_{0}(c)$ is a morphism of complexes $\left(M e_{t}, u_{\mid M e_{t}}^{0}\right) \longrightarrow\left(M e_{s}[|c|], \tau\left(u_{\mid M e_{s}}^{0}\right) \tau^{-1}\right)$.

The complex $\left(M e_{t}, u_{\mid M e_{t}}^{0}\right)$ is acyclic, so it is homotopically trivial. Then, so is the morphism $-\tau f_{0}(c)$. Hence, there is a homogeneous morphism $\underline{h}_{0}^{1}(c) \in$ $\operatorname{Hom}_{\mathrm{GMod}-k}^{-1}\left(M e_{t}, M e_{s}[|c|]\right)$ such that

$$
-\tau f_{0}(c)=\tau u^{0} \tau^{-1} \underline{h}_{0}^{1}(c)+\underline{h}_{0}^{1}(c) u^{0} .
$$

Then, we have the homogeneous morphism $\widehat{h}_{0}^{1}(c):=\tau^{-1} \underline{h}_{0}^{1}(c): M e_{t} \longrightarrow M e_{s}$, with degree $|c|-1$, such that $-f_{0}(c)=u^{0} \widehat{h}_{0}^{1}(c)+\widehat{h}_{0}^{1}(c) u^{0}$. Hence,

$$
\widehat{\Delta}_{0}^{1}(c):=u_{1}^{1}(c) h^{0}+h^{0} u_{1}^{1}(c)+u^{0} \widehat{h}_{0}^{1}(c)+\widehat{h}_{0}^{1}(c) u^{0}=0 .
$$

We have defined $\widehat{h}_{0}^{1}(c)$ for any $c \in \mathbb{B}_{1}^{v}$ such that the preceding equality holds. Then, we can consider the homogeneous morphism

$$
\widehat{h}_{0}^{1}: Z\left(\bar{C}_{1}\right)=V_{1} \longrightarrow \operatorname{Hom}_{\mathrm{GMod}-k}(M, M)
$$

of degree -1 determined by the given values $\widehat{h}_{0}^{1}(c)$ on the basic elements $c \in \mathbb{B}_{1}^{v}$. Thus $\widehat{h}_{0}^{1} \in \operatorname{Hom}_{\text {GMod-S-S }}^{-1}\left(Z\left(\bar{C}_{1}\right), E_{M}\right)$ extends $h_{0}^{1}: \bar{C}_{0} \longrightarrow E_{M}$ and satisfies $\widehat{\Delta}_{0}^{1}(c)=0$, for all $c \in Z\left(\bar{C}_{1}\right)$.

Now, take an element $c \in \mathbb{B}_{1}^{w}(s, t)$, so $\bar{\mu}(c)=0$ and $\delta(c) \in Z\left(\bar{C}_{1}\right)$. Consider the homogeneous morphism $g_{0}(c): M e_{t} \longrightarrow M e_{s}$ of degree $|c|$ given by

$$
g_{0}(c):=\widehat{d}\left(\widehat{h}_{0}^{1}\right)(c)+u_{1}^{1}(c) h^{0}+h^{0} u_{1}^{1}(c) .
$$

Write $\lambda(c):=u_{1}^{1}(c) h^{0}+h^{0} u_{1}^{1}(c)$ and $\gamma(c):=\widehat{d}\left(\widehat{h}_{0}^{1}\right)(c)$. Since $i d_{M}=u^{0} h^{0}+h^{0} u^{0}$, we have

$$
\begin{aligned}
u^{0} \lambda(c)-\lambda(c) u^{0}= & u^{0} u^{1}(c) h^{0}+u^{0} h^{0} u^{1}(c)-u^{1}(c) h^{0} u^{0}-h^{0} u^{1}(c) u^{0} \\
= & u^{0} u^{1}(c) h^{0}+u^{1}(c)-h^{0} u^{0} u^{1}(c) \\
& -u^{1}(c)+u^{1}(c) u^{0} h^{0}-h^{0} u^{1}(c) u^{0} \\
= & {\left[u^{0} u_{1}^{1}(c)+u_{1}^{1}(c) u^{0}\right] h^{0}-h^{0}\left[u^{0} u_{1}^{1}(c)+u_{1}^{1}(c) u^{0}\right] . }
\end{aligned}
$$


Since $\delta(c) \in Z\left(\bar{C}_{1}\right)$, we have $\widehat{\Delta}_{0}^{1}(\delta(c))=0$. Then, for any homogeneous element $m \in M e_{t}$, we have

$$
\begin{aligned}
{\left[u^{0} \gamma(c)-\gamma(c) u^{0}\right](m) } & =\left[u^{0} \widehat{d}\left(\widehat{h}_{0}^{1}\right)(c)-\widehat{d}\left(\widehat{h}_{0}^{1}\right)(c) u^{0}\right](m) \\
& =\left(u^{0} \widehat{d}\left(\widehat{h}_{0}^{1}\right)(c)\right)[m]-\left(\widehat{d}\left(\widehat{h}_{0}^{1}\right)(c)\right)\left(u^{0}[m]\right) \\
& =(-1)^{|m|} u^{0} \widehat{h}_{0}^{1}(\delta(c))[m]-(-1)^{|m|+1} \widehat{h}_{0}^{1}(\delta(c))\left(u^{0}[m]\right) \\
& =(-1)^{|m|}\left[u^{0} \widehat{h}_{0}^{1}(\delta(c))+\widehat{h}_{0}^{1}(\delta(c)) u^{0}\right](m) \\
& =(-1)^{|m|+1}\left[u^{1}(\delta(c)) h^{0}+h^{0} u^{1}(\delta(c))\right](m) \\
& =\left[\widehat{d}\left(u_{1}^{1}\right)(c) h^{0}-h^{0} \widehat{d}\left(u_{1}^{1}\right)(c)\right](m) .
\end{aligned}
$$

Thus, $u^{0} \gamma(c)-\gamma(c) u^{0}=\left[\widehat{d}\left(u_{1}^{1}\right) h^{0}-h^{0} \widehat{d}\left(u_{1}^{1}\right)\right](c)$. Then, from (8.1) (A), we have

$$
\begin{aligned}
u^{0} g_{0}(c)-g_{0}(c) u^{0}= & u^{0} \lambda(c)-\lambda(c) u^{0}+u^{0} \gamma(c)-\gamma(c) u^{0} \\
= & {\left[\widehat{d}\left(u_{1}^{1}\right)(c)+u^{0} u_{1}^{1}(c)+u_{1}^{1}(c) u^{0}\right] h^{0} } \\
& -h_{0}\left[\widehat{d}\left(u_{1}^{1}\right)(c)+u^{0} u_{1}^{1}(c)+u_{1}^{1}(c) u^{0}\right]=0 .
\end{aligned}
$$

Thus $\tau g_{0}(c)$ is a morphism of complexes $\left.\left(M e_{t}, u_{\mid M e_{t}}^{0}\right) \longrightarrow\left(M e_{s}[|c|]\right), \tau\left(u_{\mid M e_{s}}^{0}\right) \tau^{-1}\right)$. Proceeding as before, we get a homogeneous morphism $\widetilde{h}_{0}^{1}(c): M e_{t} \longrightarrow M e_{s}$ with degree $|c|-1$ such that

$$
g_{0}(c)=-u^{0} \widetilde{h}_{0}^{1}(c)-\widetilde{h}_{0}^{1}(c) u^{0} .
$$

Then, we have the equality

$$
\widetilde{\Delta}_{0}^{1}(c):=\widehat{d}\left(\widehat{h}_{0}^{1}\right)(c)+u_{1}^{1}(c) h^{0}+h^{0} u_{1}^{1}(c)+u^{0} \widetilde{h}_{0}^{1}(c)+\widetilde{h}_{0}^{1}(c) u^{0}=0 .
$$

We have the homogeneous morphism $\widetilde{h}_{0}^{1}: W_{1} \longrightarrow \operatorname{Hom}_{\mathrm{GMod}-k}(M, M)$ of degree -1 determined by the given values $\widetilde{h}_{0}^{1}(c)$ on the basic elements $c \in \mathbb{B}_{1}^{w}$. Then, the morphisms $\widehat{h}_{0}^{1}$ and $\widetilde{h}_{0}^{1}$ determine a homogeneous morphism $h_{1}^{1}: \bar{C}_{1}=Z\left(\bar{C}_{1}\right) \oplus$ $W_{1} \longrightarrow E_{M}$ of degree -1 such that the equation $\Delta_{0}^{1}(c)=0$ is satisfied for all $c \in \bar{C}_{1}$, because either $\widehat{\Delta}_{0}^{1}(c)=0$ or $\widetilde{\Delta}_{0}^{1}(c)$ hold on basic elements $c \in \mathbb{B}_{1}$. Clearly, $h_{1}^{1}$ extends $h_{0}^{1}$.

Step 2: The construction of $h_{i+1}^{1}: \bar{C}_{i+1} \longrightarrow E_{M}$, from $h_{i}^{1}: \bar{C}_{i} \longrightarrow E_{M}$.

Assume we have already defined the homogeneous morphism $h_{i}^{1}: \bar{C}_{i} \longrightarrow E_{M}$ of degree -1 such that $\Delta_{i-1}^{1}(c)=0$ holds for all $c \in \bar{C}_{i}$.

Given a basic element $c \in \mathbb{B}_{i+1}^{v}(s, t)$, we consider the homogeneous morphism $f_{i}(c): M e_{t} \longrightarrow M e_{s}$ of degree $|c|$ given by

$$
f_{i}(c):=u_{i+1}^{1}(c) h^{0}+h^{0} u_{i+1}^{1}(c)+\left(u_{i}^{1} \cdot h_{i}^{1}\right)(c)+\left(h_{i}^{1} \cdot u_{i}^{1}\right)(c) .
$$

Write $\lambda(c):=u_{i+1}^{1}(c) h^{0}+h^{0} u_{i+1}^{1}(c)$ and $\rho(c)=\left(u_{i}^{1} \cdot h_{i}^{1}\right)(c)+\left(h_{i}^{1} \cdot u_{i}^{1}\right)(c)$. Then, from (8.1) (A), we have

$$
\begin{aligned}
u^{0} \lambda(c)-\lambda(c) u^{0} & =u^{0} u^{1}(c) h^{0}+u^{0} h^{0} u^{1}(c)-u^{1}(c) h^{0} u^{0}-h^{0} u^{1}(c) u^{0} \\
& =u^{0} u^{1}(c) h^{0}-h^{0} u^{0} u^{1}(c)+u^{1}(c)+u^{1}(c) u^{0} h^{0}-u^{1}(c)-h^{0} u^{1}(c) u^{0} \\
& =\left[u^{0} u^{1}(c)+u^{1}(c) u^{0}\right] h^{0}-h^{0}\left[u^{0} u^{1}(c)+u^{1}(c) u^{0}\right] \\
& =-\left(\widehat{d}\left(u_{i+1}^{1}\right) h^{0}\right)(c)-\left(u_{i}^{1} \cdot u_{i}^{1}\right)(c) h^{0}+\left(h^{0} \widehat{d}\left(u_{i+1}^{1}\right)\right)(c)+h^{0}\left(u_{i}^{1} \cdot u_{i}^{1}\right)(c) \\
& =\left(h^{0} \widehat{d}\left(u_{i+1}^{1}\right)\right)(c)+\left(h^{0} u_{i}^{1} \cdot u_{i}^{1}\right)(c)-\left(\widehat{d}\left(u_{i+1}^{1}\right) h^{0}\right)(c)-\left(u_{i}^{1} \cdot u_{i}^{1} h^{0}\right)(c)
\end{aligned}
$$


and, since $c \in Z\left(\bar{C}_{i+1}\right)$, we obtain

$$
u^{0} \lambda(c)-\lambda(c) u^{0}=\left(h^{0} u_{i}^{1} \cdot u_{i}^{1}\right)(c)-\left(u_{i}^{1} \cdot u_{i}^{1} h^{0}\right)(c) .
$$

Moreover, we have

$$
\begin{aligned}
u^{0} \rho(c)-\rho(c) u^{0} & =u^{0}\left(u_{i}^{1} \cdot h_{i}^{1}\right)(c)+u^{0}\left(h_{i}^{1} \cdot u_{i}^{1}\right)(c)-\left(u_{i}^{1} \cdot h_{i}^{1}\right)(c) u^{0}-\left(h_{i}^{1} \cdot u_{i}^{1}\right)(c) u^{0} . \\
& =\left(u^{0} u_{i}^{1} \cdot h_{i}^{1}\right)(c)+\left(u^{0} h_{i}^{1} \cdot u_{i}^{1}\right)(c)-\left(u_{i}^{1} \cdot h_{i}^{1} u^{0}\right)(c)-\left(h_{i}^{1} \cdot u_{i}^{1} u^{0}\right)(c) .
\end{aligned}
$$

Now, we have $\Delta_{i-1}^{1}=0$. That is the following equality of morphisms from $\bar{C}_{i}$ to $E_{M}$ holds

$$
\widehat{d}\left(h_{i}^{1}\right)+u^{0} h_{i}^{1}+u_{i}^{1} h^{0}+u_{i-1}^{1} \cdot h_{i-1}^{1}+h^{0} u_{i}^{1}+h_{i}^{1} u^{0}+h_{i-1}^{1} \cdot u_{i-1}^{1}=0 .
$$

Mutiplying the equation $\Delta_{i-1}^{1}=0$ on the right by $u_{i}^{1}$, we have the following equality of morphisms from $\bar{C}_{i+1}$ to $E_{M}$

$u^{0} h_{i}^{1} \cdot u_{i}^{1}=-\widehat{d}\left(h_{i}^{1}\right) \cdot u_{i}^{1}-u_{i}^{1} h^{0} \cdot u_{i}^{1}-\left[u_{i-1}^{1} \cdot h_{i-1}^{1}\right] \cdot u_{i}^{1}-h^{0} u_{i}^{1} \cdot u_{i}^{1}-h_{i}^{1} u^{0} \cdot u_{i}^{1}-\left[h_{i-1}^{1} \cdot u_{i-1}^{1}\right] \cdot u_{i}^{1}$.

Evaluating at our fixed element $c$, we obtain

$$
\begin{aligned}
\left(u^{0} h_{i}^{1} \cdot u_{i}^{1}\right)(c)= & -\left(\widehat{d}\left(h_{i}^{1}\right) \cdot u_{i}^{1}\right)(c)-\left(u_{i}^{1} h^{0} \cdot u_{i}^{1}\right)(c)-\left(\left[u_{i-1}^{1} \cdot h_{i-1}^{1}\right] \cdot u_{i}^{1}\right)(c) \\
& -\left(h^{0} u_{i}^{1} \cdot u_{i}^{1}\right)(c)-\left(h_{i}^{1} u^{0} \cdot u_{i}^{1}\right)(c)-\left(\left[h_{i-1}^{1} \cdot u_{i-1}^{1}\right] \cdot u_{i}^{1}\right)(c) .
\end{aligned}
$$

Similarly, multiplying the equation $\Delta_{i-1}^{1}=0$ on the left by $u_{i}^{1}$, we have

$-u_{i}^{1} \cdot h_{i}^{1} u^{0}=u_{i}^{1} \cdot \widehat{d}\left(h_{i}^{1}\right)+u_{i}^{1} \cdot u^{0} h_{i}^{1}+u_{i}^{1} \cdot u_{i}^{1} h^{0}+u_{i}^{1} \cdot\left[u_{i-1}^{1} \cdot h_{i-1}^{1}\right]+u_{i}^{1} \cdot h^{0} u_{i}^{1}+u_{i}^{1} \cdot\left[h_{i-1}^{1} \cdot u_{i-1}^{1}\right]$.

Evaluating at the element $c$, we get

$$
\begin{aligned}
-\left(u_{i}^{1} \cdot h_{i}^{1} u^{0}\right)(c)= & \left(u_{i}^{1} \cdot \widehat{d}\left(h_{i}^{1}\right)\right)(c)+\left(u_{i}^{1} \cdot u^{0} h_{i}^{1}\right)(c)+\left(u_{i}^{1} \cdot u_{i}^{1} h^{0}\right)(c) \\
& +\left(u_{i}^{1} \cdot\left[u_{i-1}^{1} \cdot h_{i-1}^{1}\right]\right)(c)+\left(u_{i}^{1} \cdot h^{0} u_{i}^{1}\right)(c)+\left(u_{i}^{1} \cdot\left[h_{i-1}^{1} \cdot u_{i-1}^{1}\right]\right)(c) .
\end{aligned}
$$

Then, since $u_{i}^{1} h^{0} \cdot u_{i}^{1}=u_{i}^{1} \cdot h^{0} u_{i}^{1}$, we have

$$
\begin{aligned}
u^{0} \rho(c)-\rho(c) u^{0}= & \left(u^{0} u_{i}^{1} \cdot h_{i}^{1}\right)(c)-\left(h_{i}^{1} \cdot u_{i}^{1} u^{0}\right)(c) \\
& -\left(\widehat{d}\left(h_{i}^{1}\right) \cdot u_{i}^{1}\right)(c)-\left(\left[u_{i-1}^{1} \cdot h_{i-1}^{1}\right] \cdot u_{i}^{1}\right)(c) \\
& -\left(h^{0} u_{i}^{1} \cdot u_{i}^{1}\right)(c)-\left(h_{i}^{1} u^{0} \cdot u_{i}^{1}\right)(c)-\left(\left[h_{i-1}^{1} \cdot u_{i-1}^{1}\right] \cdot u_{i}^{1}\right)(c) \\
& +\left(u_{i}^{1} \cdot \widehat{d}\left(h_{i}^{1}\right)\right)(c)+\left(u_{i}^{1} \cdot u^{0} h_{i}^{1}\right)(c)+\left(u_{i}^{1} \cdot u_{i}^{1} h^{0}\right)(c) \\
& +\left(u_{i}^{1} \cdot\left[u_{i-1}^{1} \cdot h_{i-1}^{1}\right]\right)(c)+\left(u_{i}^{1} \cdot\left[h_{i-1}^{1} \cdot u_{i-1}^{1}\right]\right)(c) .
\end{aligned}
$$

Moreover, from the associativity of (8.2), we have

$$
\begin{aligned}
u^{0} \rho(c)-\rho(c) u^{0}= & -\left(\widehat{d}\left(h_{i}^{1}\right) \cdot u_{i}^{1}\right)(c)-\left(h_{i}^{1} \cdot\left[u^{0} u_{i}^{1}+u_{i}^{1} u^{0}+u_{i-1}^{1} \cdot u_{i-1}^{1}\right]\right)(c) \\
& +\left(u_{i}^{1} \cdot \widehat{d}\left(h_{i}^{1}\right)\right)(c)+\left(\left[u^{0} u_{i}^{1}+u_{i}^{1} u^{0}+u_{i-1}^{1} \cdot u_{i-1}^{1}\right] \cdot h_{i}^{1}\right)(c) \\
& +\left(u_{i}^{1} \cdot u_{i}^{1} h^{0}\right)(c)-\left(h^{0} u_{i}^{1} \cdot u_{i}^{1}\right)(c) .
\end{aligned}
$$

Since $h_{i}^{1}, u_{i}^{1}$ are defined on $\bar{C}_{i}$, they are defined on $\bar{C}_{i-1}+Z\left(\bar{C}_{i}\right)$, then the morphisms $\widehat{d}\left(h_{i}^{1}\right)$ and $\widehat{d}\left(u_{i}^{1}\right)$ are defined on $\bar{C}_{i}$. Moreover, the morphisms $h_{i}^{1}$ and 
$u_{i}^{1}$ are defined on $\bar{C}_{i}$, hence their product $h_{i}^{1} \cdot u_{i}^{1}$ is defined on $\bar{C}_{i+1}$, hence it is defined on $\bar{C}_{i}+Z\left(\bar{C}_{i+1}\right)$. Therefore, by the Leibniz formula of (8.2), we have

$$
0=\widehat{d}\left(h_{i}^{1} \cdot u_{i}^{1}\right)(c)=\left[\widehat{d}\left(h_{i}^{1}\right) \cdot u_{i}^{1}-h_{i}^{1} \cdot \widehat{d}\left(u_{i}^{1}\right)\right](c),
$$

where the left equality is due to the fact that $c \in \mathbb{B}_{i+1}^{v}(s, t) \subseteq V_{i+1} \subseteq Z\left(\bar{C}_{i+1}\right)$. Thus, $\left(\widehat{d}\left(h_{i}^{1}\right) \cdot u_{i}^{1}\right)(c)=\left(h_{i}^{1} \cdot \widehat{d}\left(u_{i}^{1}\right)\right)(c)$ and, similarly, we have $\left(\widehat{d}\left(u_{i}^{1}\right) \cdot h_{i}^{1}\right)(c)=$ $\left(u_{i}^{1} \cdot \widehat{d}\left(h_{i}^{1}\right)\right)(c)$. Then, we have

$$
\begin{aligned}
u^{0} \rho(c)-\rho(c) u^{0}= & -\left(h_{i}^{1} \cdot\left[\widehat{d}\left(u_{i}^{1}\right)+u^{0} u_{i}^{1}+u_{i}^{1} u^{0}+u_{i-1}^{1} \cdot u_{i-1}^{1}\right]\right)(c) \\
& +\left(\left[\widehat{d}\left(u_{i}^{1}\right)+u^{0} u_{i}^{1}+u_{i}^{1} u^{0}+u_{i-1}^{1} \cdot u_{i-1}^{1}\right] \cdot h_{i}^{1}\right)(c) \\
& +\left(u_{i}^{1} \cdot u_{i}^{1} h^{0}\right)(c)-\left(h^{0} u_{i}^{1} \cdot u_{i}^{1}\right)(c) .
\end{aligned}
$$

From (8.1) (A), we know that $\widehat{d}\left(u_{i}^{1}\right)+u^{0} u_{i}^{1}+u_{i}^{1} u^{0}+u_{i-1}^{1} \cdot u_{i-1}^{1}=0$ on $\bar{C}_{i}$. It follows that $u^{0} f_{i}(c)-f_{i}(c) u^{0}=u^{0} \lambda(c)-\lambda(c) u^{0}+u^{0} \rho(c)-\rho(c) u^{0}=0$. As before, we have a morphism of complexes $-\tau f_{i}(c):\left(M e_{t}, u_{\mid M e_{t}}^{0}\right) \longrightarrow\left(M e_{s}[|c|], \tau u_{\mid M e_{s}}^{0} \tau^{-1}\right)$ which is homotopically trivial. As a consequence, there is a homogeneous mor$\operatorname{phism} \underline{h}_{i}^{1}(c) \in \operatorname{Hom}_{\mathrm{GMod}-k}^{-1}\left(M e_{t}, M e_{s}[|c|]\right)$ such that

$$
-\tau f_{i}(c)=\tau u^{0} \tau^{-1} \underline{h}_{i}^{1}(c)+\underline{h}_{i}^{1}(c) u^{0} .
$$

The morphism $\widehat{h}_{i}^{1}(c):=\tau^{-1} \underline{h}_{i}^{1}(c): M e_{t} \longrightarrow M e_{s}$ is homogeneous of degree $|c|-1$ such that $-f_{i}(c)=u^{0} \widehat{h}_{i}^{1}(c)+\widehat{h}_{i}^{1}(c) u^{0}$. Hence,

$\widehat{\Delta}_{i}^{1}(c):=u_{i+1}^{1}(c) h^{0}+h^{0} u_{i+1}^{1}(c)+u^{0} \widehat{h}_{i}^{1}(c)+\widehat{h}_{i}^{1}(c) u^{0}+\left(u_{i}^{1} \cdot h_{i}^{1}\right)(c)+\left(h_{i}^{1} \cdot u_{i}^{1}\right)(c)=0$.

We have defined $\widehat{h}_{i}^{1}(c)$ for any $c \in \mathbb{B}_{i+1}^{v}$ such that the preceding equality holds. Then, we can consider the homogeneous morphism

$$
\widehat{h}_{i}^{1}: \bar{C}_{i} \oplus V_{i+1} \longrightarrow \operatorname{Hom}_{\mathrm{GMod}-k}(M, M)
$$

of degree -1 determined by the given values $\widehat{h}_{i}^{1}(c)$ on the basic elements $c \in \mathbb{B}_{i+1}^{v}$ and by $\widehat{h}_{i}^{1}(c):=h_{i}^{1}(c)$ on the basic elements $c \in \mathbb{B}_{i}$. Therefore, the morphism $\widehat{h}_{i}^{1} \in \operatorname{Hom}_{\text {GMod-S-S }}^{-1}\left(\bar{C}_{i}+Z\left(\bar{C}_{i+1}\right), E_{M}\right)$ extends $h_{i}^{1}: \bar{C}_{i} \longrightarrow E_{M}$ and satisfies $\widehat{\Delta}_{i}^{1}(c)=0$, for all $c \in Z\left(\bar{C}_{i+1}\right)$.

Now, take an element $c \in \mathbb{B}_{i+1}^{w}(s, t)$. Consider the homogeneous morphism $g_{i}(c): M e_{t} \longrightarrow M e_{s}$ of degree $|c|$ given by

$$
g_{i}(c):=\widehat{d}\left(\widehat{h}_{i}^{1}\right)(c)+u_{i+1}^{1}(c) h^{0}+h^{0} u_{i+1}^{1}(c)+\left(u_{i}^{1} \cdot h_{i}^{1}\right)(c)+\left(h_{i}^{1} \cdot u_{i}^{1}\right)(c) .
$$

Write $\lambda(c):=u_{i+1}^{1}(c) h^{0}+h^{0} u_{i+1}^{1}(c), \rho(c)=\left(u_{i}^{1} \cdot h_{i}^{1}\right)(c)+\left(h_{i}^{1} \cdot u_{i}^{1}\right)(c)$ and $\gamma(c)=\widehat{d}\left(\widehat{h}_{i}^{1}\right)(c)$. Then, the same calculations used at the beginining of the preceding case show that the following two equalities hold

$u^{0} \lambda(c)-\lambda(c) u^{0}=\left(h^{0} \widehat{d}\left(u_{i+1}^{1}\right)\right)(c)+\left(h^{0} u_{i}^{1} \cdot u_{i}^{1}\right)(c)-\left(\widehat{d}\left(u_{i+1}^{1}\right) h^{0}\right)(c)-\left(u_{i}^{1} \cdot u_{i}^{1} h^{0}\right)(c)$ 
and

$$
\begin{aligned}
u^{0} \rho(c)-\rho(c) u^{0}= & -\left(\widehat{d}\left(h_{i}^{1}\right) \cdot u_{i}^{1}\right)(c)-\left(h_{i}^{1} \cdot\left[u^{0} u_{i}^{1}+u_{i}^{1} u^{0}+u_{i-1}^{1} \cdot u_{i-1}^{1}\right]\right)(c) \\
& +\left(u_{i}^{1} \cdot \widehat{d}\left(h_{i}^{1}\right)\right)(c)+\left(\left[u^{0} u_{i}^{1}+u_{i}^{1} u^{0}+u_{i-1}^{1} \cdot u_{i-1}^{1}\right] \cdot h_{i}^{1}\right)(c) \\
& +\left(u_{i}^{1} \cdot u_{i}^{1} h^{0}\right)(c)-\left(h^{0} u_{i}^{1} \cdot u_{i}^{1}\right)(c) .
\end{aligned}
$$

Applying again Leibniz formula of (8.2), we have

$$
\left(\widehat{d}\left(h_{i}^{1}\right) \cdot u_{i}^{1}\right)(c)=\left(h_{i}^{1} \cdot \widehat{d}\left(u_{i}^{1}\right)\right)(c)+\widehat{d}\left(h_{i}^{1} \cdot u_{i}^{1}\right)(c)
$$

and

$$
\left(u_{i}^{1} \cdot \widehat{d}\left(h_{i}^{1}\right)\right)(c)=\left(\widehat{d}\left(u_{i}^{1}\right) \cdot h_{i}^{1}\right)(c)-\widehat{d}\left(u_{i}^{1} \cdot h_{i}^{1}\right)(c) .
$$

Thus,

$$
\begin{aligned}
u^{0} \rho(c)-\rho(c) u^{0}= & -\left(h_{i}^{1} \cdot\left[\widehat{d}\left(u_{i}^{1}\right)+u^{0} u_{i}^{1}+u_{i}^{1} u^{0}+u_{i-1}^{1} \cdot u_{i-1}^{1}\right]\right)(c) \\
& +\left(\left[\widehat{d}\left(u_{i}^{1}\right)+u^{0} u_{i}^{1}+u_{i}^{1} u^{0}+u_{i-1}^{1} \cdot u_{i-1}^{1}\right] \cdot h_{i}^{1}\right)(c) \\
& +\left(u_{i}^{1} \cdot u_{i}^{1} h^{0}\right)(c)-\left(h^{0} u_{i}^{1} \cdot u_{i}^{1}\right)(c) \\
& -\widehat{d}\left[h_{i}^{1} \cdot u_{i}^{1}+u_{i}^{1} \cdot h_{i}^{1}\right](c) .
\end{aligned}
$$

From (8.1) (A), we obtain

$$
u^{0} \rho(c)-\rho(c) u^{0}=\left(u_{i}^{1} \cdot u_{i}^{1} h^{0}\right)(c)-\left(h^{0} u_{i}^{1} \cdot u_{i}^{1}\right)(c)-\widehat{d}\left[h_{i}^{1} \cdot u_{i}^{1}+u_{i}^{1} \cdot h_{i}^{1}\right](c) .
$$

Since $u^{0} \gamma(c)-\gamma(c) u^{0}=u^{0} \widehat{d}\left(\widehat{h}_{i}^{1}\right)(c)-\widehat{d}\left(\widehat{h}_{i}^{1}\right)(c) u^{0}$, we have

$$
\begin{aligned}
u^{0} g_{i}(c)-g_{i}(c) u^{0}= & u^{0} \lambda(c)-\lambda(c) u^{0}+u^{0} \rho(c)-\rho(c) u^{0}+u^{0} \gamma(c)-\gamma(c) u^{0} \\
= & {\left[u^{0} \widehat{d}\left(\widehat{h}_{i}^{1}\right)-\widehat{d}\left(\widehat{h}_{i}^{1}\right) u^{0}\right](c)-\widehat{d}\left[h_{i}^{1} \cdot u_{i}^{1}+u_{i}^{1} \cdot h_{i}^{1}\right](c) } \\
& +h^{0} \widehat{d}\left(u_{i+1}^{1}\right)(c)-\widehat{d}\left(u_{i+1}^{1}\right) h^{0}(c) .
\end{aligned}
$$

Moreover, $\delta(c) \in Z\left(\bar{C}_{i+1}\right)$, so $\widehat{\Delta}_{i}^{1}(\delta(c))=0$. Hence,

$$
\begin{aligned}
\widehat{d}\left[u_{i}^{1} \cdot h_{i}^{1}+h_{i}^{1} \cdot u_{i}^{1}\right](c)[m]= & (-1)^{|m|+1}\left[u_{i}^{1} \cdot h_{i}^{1}+h_{i}^{1} \cdot u_{i}^{1}\right](\delta(c))[m] \\
= & (-1)^{|m|}\left[u_{i+1}^{1} h^{0}+h^{0} u_{i+1}^{1}+u^{0} \widehat{h}_{i}^{1}+\widehat{h}_{i}^{1} u^{0}\right](\delta(c))[m] \\
= & (-1)^{|m|}\left(u_{i+1}^{1} h^{0}\right)(\delta(c))[m]+(-1)^{|m|}\left(h^{0} u_{i+1}^{1}\right)(\delta(c))[m] \\
& +(-1)^{|m|}\left(u^{0} \widehat{h}_{i}^{1}\right)(\delta(c))[m]+(-1)^{|m|}\left(\widehat{h}_{i}^{1} u^{0}\right)(\delta(c))[m] \\
= & {\left[-\widehat{d}\left(u_{i+1}^{1} h^{0}\right)-\widehat{d}\left(h^{0} u_{i+1}^{1}\right)-\widehat{d}\left(u^{0} \widehat{h}_{i}^{1}\right)-\widehat{d}\left(\widehat{h}_{i}^{1} u^{0}\right)\right](c)[m] . }
\end{aligned}
$$

Thus,

$$
\widehat{d}\left[u_{i}^{1} \cdot h_{i}^{1}+h_{i}^{1} \cdot u_{i}^{1}\right](c)=\left[-\widehat{d}\left(u_{i+1}^{1}\right) h^{0}+h^{0} \widehat{d}\left(u_{i+1}^{1}\right)+u^{0} \widehat{d}\left(\widehat{h}_{i}^{1}\right)-\widehat{d}\left(\widehat{h}_{i}^{1}\right) u^{0}\right](c) .
$$

Therefore, $u^{0} g_{i}(c)-g_{i}(c) u^{0}=0$ and $\tau g_{i}(c)$ is a morphism of complexes $\left.\left(M e_{t}, u_{\mid M e_{t}}^{0}\right) \longrightarrow\left(M e_{s}[|c|]\right), \tau\left(u_{\mid M e_{s}}^{0}\right) \tau^{-1}\right)$. Proceeding as before, we get a homogeneous morphism $\widetilde{h}_{i}^{1}(c): M e_{t} \longrightarrow M e_{s}$ with degree $|c|-1$ such that

$$
g_{i}(c)=-u^{0} \widetilde{h}_{i}^{1}(c)-\widetilde{h}_{i}^{1}(c) u^{0} .
$$


Then, we have the equality

$\widetilde{\Delta}_{i}^{1}(c):=\widehat{d}\left(\widehat{h}_{i}^{1}\right)(c)+u_{i+1}^{1}(c) h^{0}+h^{0} u_{i+1}^{1}(c)+\left(u_{i}^{1} \cdot h_{i}^{1}\right)(c)+\left(h_{i}^{1} \cdot u_{i}^{1}\right)(c)+u^{0} \widetilde{h}_{i}^{1}(c)+\widetilde{h}_{i}^{1}(c) u^{0}=0$.

Consider the homogeneous morphism $\widetilde{h}_{i}^{1}: W_{i+1} \longrightarrow \operatorname{Hom}_{\mathrm{GMod}-k}(M, M)$ of degree -1 determined by the given values $\widetilde{h}_{i}^{1}(c)$ on the basic elements $c \in \mathbb{B}_{i+1}^{w}$. Then, the morphisms $h_{i}^{1}, \widehat{h}_{i}^{1}$ and $\widetilde{h}_{i}^{1}$ determine a homogeneous morphism

$$
h_{i+1}^{1}: \bar{C}_{i+1}=\bar{C}_{i} \oplus V_{i+1} \oplus W_{i+1} \longrightarrow E_{M}
$$

of degree -1 such that the equation $\Delta_{i}^{1}(c)=0$ is satisfied for all $c \in \bar{C}_{i+1}$, because either $\Delta_{i-1}^{1}(c)=0, \widehat{\Delta}_{i}^{1}(c)=0$ or $\widetilde{\Delta}_{i}^{1}(c)=0$ hold on basic elements $c \in \mathbb{B}_{i+1}$. Clearly, $h_{i+1}^{1}$ extends $h_{i}^{1}$.

Theorem 8.4. Assume that $S$ is a finite product of copies of the field $k$. Let $\mathcal{B}$ be a triangular differential graded $S$-bocs and $f=\left(f^{0}, f^{1}\right):\left(M, u_{M}\right) \longrightarrow\left(N, u_{N}\right)$ a morphism in $\mathrm{TGMod}^{0}-\mathcal{B}$. Then $f$ is a homotopy equivalence iff the morphism of complexes of right $S$-modules $f^{0}:\left(M, u_{M}^{0}\right) \longrightarrow\left(N, u_{N}^{0}\right)$ is a quasi-isomorphism.

Proof. Consider the homotopy category $\underline{\mathrm{TGMod}}^{0}-\mathcal{B}$ with its triangulated structure as in (7.16). Then, there is a triangle of the form

$$
\left(M, u_{M}\right) \stackrel{f}{\longrightarrow}\left(N, u_{N}\right) \stackrel{g}{\longrightarrow}\left(L, u_{L}\right) \longrightarrow\left(M, u_{M}\right)[1]
$$

of $\underline{\mathrm{TGMod}}^{0}-\mathcal{B}$. By definition of this triangular structure, the preceding triangle is isomorphic to a triangle of the form

$$
\left(K, u_{K}\right) \stackrel{\varphi}{\longrightarrow}\left(E, u_{E}\right) \stackrel{\underline{\gamma}}{\longrightarrow}\left(Q, u_{Q}\right) \longrightarrow\left(K, u_{K}\right)[1],
$$

where

$$
\xi \quad: \quad\left(K, u_{K}\right) \stackrel{\phi}{\longrightarrow}\left(E, u_{E}\right) \stackrel{\gamma}{\longrightarrow}\left(Q, u_{Q}\right)
$$

is an exact pair in the exact structure $\mathcal{E}$ of $\operatorname{TGMod}^{0}-\mathcal{B}$. Consider an isomorphism of triangles

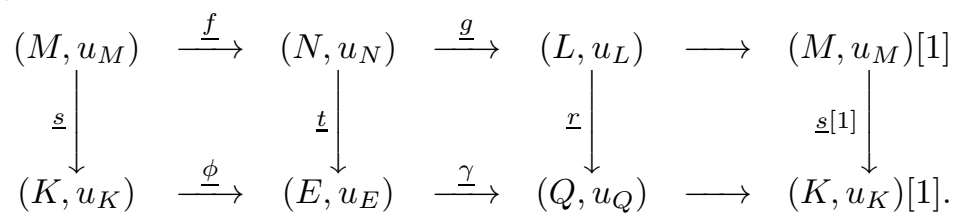

Then, the morphisms $s=\left(s^{0}, s^{1}\right):\left(M, u_{M}\right) \longrightarrow\left(K, u_{K}\right)$ and $t=\left(t^{0}, t^{1}\right)$ : $\left(N, u_{N}\right) \longrightarrow\left(E, u_{E}\right)$ are homotopy equivalences such that $\phi s$ is homotopic to $t f$. Therefore, the morphisms of complexes $\phi^{0} s^{0}$ and $t^{0} f^{0}$ from $\left(M, u_{M}^{0}\right)$ to $\left(E, u_{E}^{0}\right)$ are homotopic. As a consequence

$$
H^{i}\left(\phi^{0}\right) H^{i}\left(s^{0}\right)=H^{i}\left(t^{0}\right) H^{i}\left(f^{0}\right), \text { for all } i \in \mathbb{Z} .
$$

Since $H^{i}\left(s^{0}\right)$ and $H^{i}\left(t^{0}\right)$ are isomorphisms for all $i \in \mathbb{Z}$, then $H^{i}\left(f^{0}\right)$ is an isomorphism if and only if $H^{i}\left(\phi^{0}\right)$ is an isomorphism. Thus $f^{0}$ is a quasiisomorphism iff $\phi^{0}$ is a quasi-isomorphism. Clearly, $f$ is a homotopy equivalence iff $\phi$ is so. 
Since the exact pair $\xi$ belongs to $\mathcal{E}$, we have the exact sequence of graded right $S$-modules

$$
0 \longrightarrow K \stackrel{\phi^{0}}{\longrightarrow} E \stackrel{\gamma^{0}}{\longrightarrow} Q \longrightarrow 0 .
$$

Then, we have the exact sequence of complexes of right $S$-modules

$$
0 \longrightarrow\left(K, u_{K}^{0}\right) \stackrel{\phi^{0}}{\longrightarrow}\left(E, u_{E}^{0}\right) \stackrel{\gamma^{0}}{\longrightarrow}\left(Q, u_{Q}^{0}\right) \longrightarrow 0 .
$$

In the homology long exact sequence associated to the preceding exact sequence of complexes, we see that $H^{i}\left(\phi^{0}\right)$ is an isomorphism for all $i \in \mathbb{Z}$ iff $H^{i}\left(Q, u_{Q}^{0}\right)=0$ for all $i \in \mathbb{Z}$. So $\phi^{0}$ is a quasi-isomorphism iff $\left(Q, u_{Q}^{0}\right)$ is acyclic. Then, by (8.3), we obtain that $\phi^{0}$ is a quasi-isomorphism iff $\left(Q, u_{Q}\right)$ is homotopically trivial in $\operatorname{TGMod}^{0}-\mathcal{B}$, which is equivalent to the fact that $\underline{\phi}:\left(K, u_{K}\right) \longrightarrow\left(E, u_{E}\right)$ is an isomorphism in $\underline{\mathrm{TGMod}}^{0}-\mathcal{B}$.

\section{The Frobenius category of $A_{\infty}$-modules}

Given a fixed $A_{\infty}$-algebra $A$, denote by $\mathcal{B}_{A}$ the differential tensor $S$-coalgebra (or differential tensor $S$-bocs) $\mathcal{B}_{A}=\left(T_{S}(A[1]), \mu, \epsilon, \delta\right)$ given by the bar construction. In order to describe precisely the connection of the category $\operatorname{Mod}_{\infty}-A$ of right $A_{\infty}$-modules over $A$ with the category TGMod- $\mathcal{B}_{A}$ of twisted $\mathcal{B}_{A}$-modules, it is convenient to introduce the following categories.

Definition 9.1. We will denote with GMod- $A$ the following $k$-category. Its class of objects coincides with the class of objects of GMod- $S$. Given two graded right $S$-modules $M$ and $N$, and $d \in \mathbb{Z}$, a homogeneous morphism $f: M \longrightarrow N$ of degree $d$ in GMod- $A$ is a collection of morphisms $f=\left\{f_{n}\right\}_{n \in \mathbb{N}}$, where each

$$
f_{n}: M \otimes_{S} A^{\otimes(n-1)} \longrightarrow N
$$

is a homogeneous morphism of graded right $S$-modules of degree $\left|f_{n}\right|=d+1-n$. We denote by $\operatorname{Hom}_{\text {GMod- } A}^{d}(M, N)$ the space of homogeneous morphisms from $M$ to $N$ in GMod- $A$, and we make

$$
\operatorname{Hom}_{\mathrm{GMod}-A}(M, N)=\bigoplus_{d \in \mathbb{Z}} \operatorname{Hom}_{\mathrm{GMod}-A}^{d}(M, N) .
$$

If $f \in \operatorname{Hom}_{\mathrm{GMod}-A}(M, N)$ and $g \in \operatorname{Hom}_{\mathrm{GMod}-A}(N, L)$ are homogeneous morphisms, their composition

$$
g \circ f=\left\{(g \circ f)_{n}\right\}_{n \in \mathbb{N}}: M \longrightarrow L
$$

is defined, for each $n \in \mathbb{N}$, by

$$
(g \circ f)_{n}=\sum_{\substack{r+s=n \\ r \geq 1 ; s \geq 0}}(-1)^{(|f|+r+1) s} g_{1+s}\left(f_{r} \otimes i d^{\otimes s}\right) .
$$


Given $M \in$ GMod- $A$, the identity morphism $\mathbb{I}_{M}=\left\{h_{n}\right\}: M \longrightarrow M$ is given by $h_{1}=i d_{M}$ and $h_{n}=0$, for all $n \geq 2$.

The category GMod- $A$ is a graded category with differential $\delta_{\infty}$ defined for any homogeneous $f \in \operatorname{Hom}_{\mathrm{GMod}-} A(M, N)$ by

$$
\delta_{\infty}(f)_{n}=\sum_{\substack{r+s+t=n \\ t \geq 0 ; r, s \geq 1}}(-1)^{|f|+r+s t+1} f_{n-s+1}\left(i d^{\otimes r} \otimes m_{s} \otimes i d^{\otimes t}\right),
$$

for $n \geq 1$, thus $\delta_{\infty}(f)_{1}=0$.

A morphism $f=\left\{f_{n}\right\}_{n \in \mathbb{N}}: M \longrightarrow N$ in GMod- $A$ is called strict iff $f_{n}=0$, for all $n \geq 2$.

The fact that the preceding notions give rise indeed to a differential graded category is a consequence of the following.

Proposition 9.2. Let $\mathcal{B}_{A}=\left(T_{S}(A[1]), \mu, \epsilon, \delta\right)$ denote the differential tensor $S$ bocs associated to the $A_{\infty}$-algebra $A$. Then there is an equivalence of differential graded $k$-categories

$$
\mathbb{G}: \text { GMod- } A \longrightarrow \text { GMod- } \mathcal{B}_{A} \text {. }
$$

Given $M \in$ GMod- $A$, by definition $\mathbb{G}(M)=M[1]$, so it acts as the usual translation on objects. Given two graded right $S$-modules $M, N$ and a homogeneous morphism $f=\left\{f_{n}\right\}_{n \in \mathbb{N}}: M \longrightarrow N$ in GMod-A with degree $|f|=d$, we have the family of morphisms of right $S$-modules

$$
\left\{\hat{f}_{n}: M[1] \otimes_{S} A[1]^{\otimes n} \longrightarrow N[1]\right\}_{n \geq 0}
$$

determined by the commutativity of the following diagrams

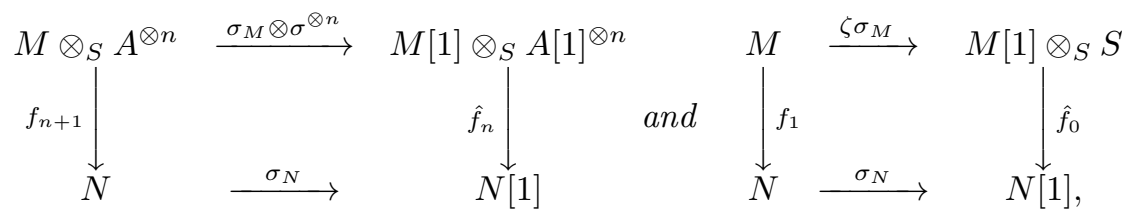

where $n$ runs in $\mathbb{N}$ and $\zeta: M[1] \longrightarrow M[1] \otimes_{S} S$ is the canonical isomorphism. Each morphism $\hat{f}_{n}$ is homogeneous with degree $\left|\hat{f}_{n}\right|=d$. The family of maps $\left\{\hat{f}_{n}\right\}_{n \geq 0}$ extends to a homogeneous morphism of right $S$-modules of degree $d$

$$
\hat{f}: M[1] \otimes T_{S}(A[1]) \longrightarrow N[1] .
$$

By definition, we have $\mathbb{G}(f)=\hat{f} \in \operatorname{Hom}_{\mathrm{GMod}-\mathcal{B}_{A}}^{d}(M[1], N[1])$.

Proof. Step 1: We have indeed a graded $k$-category GMod- $A$ and $\mathbb{G}$ is an equivalence of graded $k$-categories.

Given composable morphisms $f: M \longrightarrow N$ and $g: N \longrightarrow L$ in GMod- $A$, we know that the composition $\mathbb{G}(g) * \mathbb{G}(f)=\hat{g} * \hat{f}: M[1] \longrightarrow L[1]$ in GMod- $\mathcal{B}_{A}$ is given by the composition of maps

$$
\hat{g} * \hat{f}=\left(M[1] \otimes_{S} C \stackrel{i d_{M[1]} \otimes \mu}{\longrightarrow} M[1] \otimes_{S} C \otimes_{S} C \stackrel{\hat{f} \otimes i d_{C}}{\longrightarrow} N[1] \otimes_{S} C \stackrel{\hat{g}}{\longrightarrow} L[1]\right),
$$


where $C=T_{S}(A[1])$. Consider the restrictions $(\hat{g} * \hat{f})_{n}: M[1] \otimes A[1]^{\otimes n} \longrightarrow L[1]$, for $n \geq 0$, and let us verify that the following diagrams commute

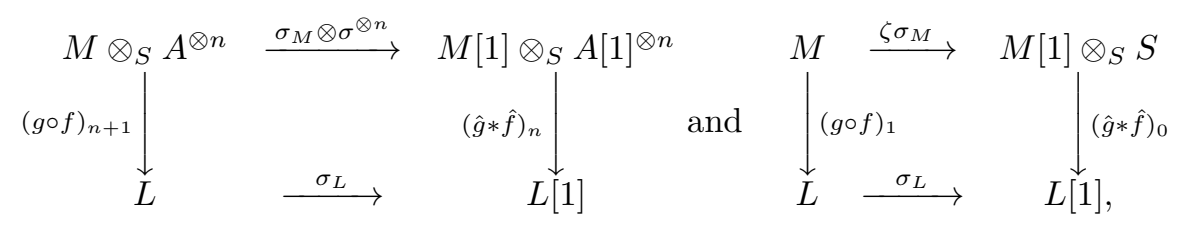

where $n$ runs in $\mathbb{N}$. For this, it is convenient to write the restriction $\mu_{n}$ : $A[1]^{\otimes n} \longrightarrow C \otimes C$ of the bar comultiplication $\mu$ on $T_{S}(A[1])$ as follows

$$
\mu_{n}=\sum_{\substack{r+s=n \\ r, s \geq 1 ;}} i d^{\otimes r} \otimes i d^{\otimes s}+1 \otimes i d^{\otimes n}+i d^{\otimes n} \otimes 1 .
$$

Then, for $n \geq 1$, we have

$$
\begin{aligned}
(\hat{g} * \hat{f})_{n}= & \sum_{r+s=n} \hat{g}_{s}\left(\hat{f}_{r}\left(i d_{M[1]} \otimes i d^{\otimes r}\right) \otimes i d^{\otimes s}\right) \\
& +\hat{g}_{n}\left(\hat{f}_{0}\left(i d_{M[1]} \otimes 1\right) \otimes i d^{\otimes n}\right)+\hat{g}_{0}\left(\hat{f}_{n}\left(i d_{M[1]} \otimes i d^{\otimes n}\right) \otimes 1\right) . \\
= & \sum_{\substack{r+s=n \\
r, s \geq 1 ;}} \hat{g}_{s}\left(\hat{f}_{r} \otimes i d^{\otimes s}\right)+\hat{g}_{n}\left(\hat{f}_{0} \zeta \otimes i d^{\otimes n}\right)+\hat{g}_{0}\left(\zeta \hat{f}_{n}\right) .
\end{aligned}
$$

Write $(\hat{g} * \hat{f})_{n}=X_{n}^{(1)}+X_{n}^{(2)}+X_{n}^{(3)}$ with $X_{n}^{(1)}:=\sum_{r+s=n} \hat{g}_{s}\left(\hat{f}_{r} \otimes i d^{\otimes s}\right)$, $X_{n}^{(2)}=\hat{g}_{0}\left(\zeta \hat{f}_{n}\right)$ and $X_{n}^{(3)}=\hat{g}_{n}\left(\hat{f}_{0} \zeta \otimes i d^{\otimes n}\right)$. We want to show that, for $n \geq 1$, we have

$$
(\hat{g} * \hat{f})_{n}\left(\sigma_{M} \otimes \sigma^{\otimes n}\right)=\sigma_{L}(g \circ f)_{n+1} .
$$

By definition of $g \circ f$, for $n \geq 1$, we have $(g \circ f)_{n}=Y_{n}^{(1)}+Y_{n}^{(2)}+Y_{n}^{(3)}$, where

$$
Y_{n}^{(1)}=\sum_{\substack{r+s=n \\ r \geq 2 ; s \geq 1}}(-1)^{(|f|+r+1) s} g_{1+s}\left(f_{r} \otimes i d^{\otimes s}\right),
$$

$Y_{n}^{(2)}=g_{1}\left(f_{n}\right), Y_{1}^{(3)}=0$ and, for $n \geq 2, Y_{n}^{(3)}=(-1)^{|f|(n-1)} g_{n}\left(f_{1} \otimes i d^{\otimes(n-1)}\right)$. 
We have

$$
\begin{aligned}
& X_{n}^{(1)}\left(\sigma_{M} \otimes \sigma^{\otimes n}\right)=\sum_{r+s=n} \hat{g}_{s}\left(\hat{f}_{r} \otimes i d^{\otimes s}\right)\left(\sigma_{M} \otimes \sigma^{\otimes n}\right) \\
& =\sum_{r+s=n} \hat{g}_{s}\left(\hat{f}_{r} \otimes i d^{\otimes s}\right)\left(\sigma_{M} \otimes \sigma^{\otimes r} \otimes \sigma^{\otimes s}\right) \\
& =\sum_{r+s=n}^{r, s \geq 1} \hat{g}_{s}\left(\hat{f}_{r}\left(\sigma_{M} \otimes \sigma^{\otimes r}\right) \otimes \sigma^{\otimes s}\right) \\
& =\sum_{r+s=n}^{r, s \geq 1} \hat{g}_{s}\left(\sigma_{N} f_{r+1} \otimes \sigma^{\otimes s}\right) \\
& =\sum_{r+s=n}^{r, s \geq 1}(-1)^{(|f|-r) s} \hat{g}_{s}\left(\sigma_{N} \otimes \sigma^{\otimes s}\right)\left(f_{r+1} \otimes i d^{\otimes s}\right) \\
& =\sum_{r+s=n}^{r, s \geq 1}(-1)^{(|f|-r) s} \sigma_{L} g_{1+s}\left(f_{r+1} \otimes i d^{\otimes s}\right) \\
& =\sum_{\substack{r^{\prime}+s=n+1 \\
r^{\prime} \geq 2 ; s \geq 1}}(-1)^{\left(|f|+r^{\prime}-1\right) s} \sigma_{L} g_{1+s}\left(f_{r^{\prime}} \otimes i d^{\otimes s}\right) \\
& =\sigma_{L} Y_{n+1}^{(\overline{1})} \text {. }
\end{aligned}
$$

We also have

$$
\begin{aligned}
X_{n}^{(2)}\left(\sigma_{M} \otimes \sigma^{\otimes n}\right) & =\hat{g}_{0} \zeta \hat{f}_{n}\left(\sigma_{M} \otimes \sigma^{\otimes n}\right) \\
& =\hat{g}_{0} \zeta \sigma_{N} f_{n+1}=\sigma_{L} g_{1}\left(f_{n+1}\right)=\sigma_{L} Y_{n+1}^{(2)}
\end{aligned}
$$

and

$$
\begin{aligned}
X_{n}^{(3)}\left(\sigma_{M} \otimes \sigma^{\otimes n}\right) & =\hat{g}_{n}\left(\hat{f}_{0} \zeta \otimes i d^{\otimes n}\right)\left(\sigma_{M} \otimes \sigma^{\otimes n}\right) \\
& =\hat{g}_{n}\left(\hat{f}_{0} \zeta \sigma_{M} \otimes \sigma^{\otimes n}\right) \\
& =\hat{g}_{n}\left(\sigma_{N} f_{1} \otimes \sigma^{\otimes n}\right) \\
& =(-1)^{|f| n} \hat{g}_{n}\left(\sigma_{N} \otimes \sigma^{\otimes n}\right)\left(f_{1} \otimes i d^{\otimes n}\right) \\
& =(-1)^{|f| n} \sigma_{L} g_{n+1}\left(f_{1} \otimes i d^{\otimes n}\right)=\sigma_{L} Y_{n+1}^{(3)} .
\end{aligned}
$$

Moreover, we have

$$
\begin{array}{rlrl}
(\hat{g} * \hat{f})_{0} \zeta \sigma_{M} & =(\hat{g} * \hat{f})_{0}\left(\sigma_{M} \otimes 1\right) & & \hat{g}\left(\hat{f} \otimes i d_{C}\right)\left(i d_{M[1]} \otimes \mu\right)\left(\sigma_{M} \otimes 1\right) \\
& =\hat{g}\left(\hat{f} \otimes i d_{C}\right)\left(\sigma_{M} \otimes 1 \otimes 1\right) & =\hat{g}\left(\hat{f}\left(\sigma_{M} \otimes 1\right) \otimes 1\right) \\
& =\hat{g}_{0} \zeta \hat{f}_{0} \zeta \sigma_{M} & & =\hat{g}_{0} \zeta \sigma_{N} f_{1} \\
& =\sigma_{L} g_{1} f_{1} & & \sigma_{L}(g \circ f)_{1} .
\end{array}
$$

From the preceding calculations, we obtain that $\mathbb{G}(g \circ f)=\mathbb{G}(g) * \mathbb{G}(f)$. Take any $M \in$ GMod- $A$ and consider the morphism $h=\left\{h_{n}\right\}_{n \in \mathbb{N}}: M \longrightarrow M$ in GMod- $A$ given by $h_{1}=i d_{M}$ and $h_{n}=0$, for $n \geq 2$. Let us show that $\mathbb{G}(h)=\mathbb{I}_{M[1]}$, the identity morphism on the object $\mathbb{G}(M) \in$ GMod- $\mathcal{B}_{A}$.

Clearly, we have $\hat{h}_{0}=\sigma_{M} h_{1}\left(\zeta \sigma_{M}\right)^{-1}=\sigma_{M} \sigma_{M}^{-1} \zeta^{-1}=\zeta^{-1}$ and, for $n \geq 1$, we have that $\hat{h}_{n}=\sigma_{M} h_{n+1}\left(\sigma_{M} \otimes \sigma^{\otimes n}\right)^{-1}=0$. This means that the components $\left(\hat{h}^{0}, \hat{h}^{1}\right)$ of the morphism $\mathbb{G}(h)=\hat{h}$ are $\left(i d_{M[1]}, 0\right)$, so $\mathbb{G}(h)=\mathbb{I}_{M[1]}$.

It is clear that the association $f \longmapsto \hat{f}$ is an isomorphism of graded vector spaces $\operatorname{Hom}_{\mathrm{GMod}-A}(M, N) \longrightarrow \operatorname{Hom}_{\mathrm{GMod}-\mathcal{B}_{A}}(M[1], N[1])$. It follows that GMod- $A$ is a graded $k$-category and that $\mathbb{G}:$ GMod- $A \longrightarrow$ GMod- $\mathcal{B}_{A}$ is an equivalence of categories. 
Step 2: We have indeed a differential $\delta_{\infty}$ on the graded category GMod-A and $\mathbb{G}$ is an equivalence of differential graded $k$-categories.

It will be enough to show that for any homogeneous morphism $f: M \longrightarrow N$ in GMod- $A$, we have

$$
\mathbb{G}\left(\delta_{\infty}(f)\right)=\widehat{\delta}(\mathbb{G}(f)) .
$$

Consider the restrictions $\widehat{\delta}(\hat{f})_{n}: M[1] \otimes A[1]^{\otimes n} \longrightarrow N[1]$, for $n \geq 0$. Let us verify that the following diagrams commute

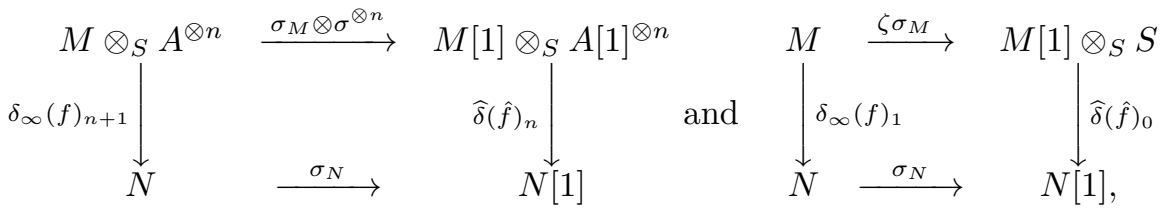

where $n$ runs in $\mathbb{N}$.

By definition, the differential $\widehat{\delta}$ of the category GMod- $\mathcal{B}_{A}$ applied to the morphism $\mathbb{G}(f)=\hat{f}$ is

$$
\widehat{\delta}(\hat{f})=(-1)^{|\hat{f}|+1} \hat{f}\left(i d_{M[1]} \otimes \delta\right) .
$$

Recall that the differential $\delta$ on the tensor $S$-coalgebra $T_{S}(A[1])$ satisfies that $\delta(S)=0$ and is determined by the morphisms of right $S$-modules

$$
\delta_{n}: A[1]^{\otimes n} \longrightarrow \bigoplus_{1 \leq s \leq n} A[1]^{\otimes(n-s+1)}
$$

given, for $n \geq 1$, by the following formula $\delta_{n}=\sum_{\substack{r+s+t=n \\ r, t \geq 0 ; s \geq 1}} i d^{\otimes r} \otimes \hat{m}_{s} \otimes i d^{\otimes t}$, where $\hat{m}_{s}$ is defined, for $s \geq 1$, by the following commutative square

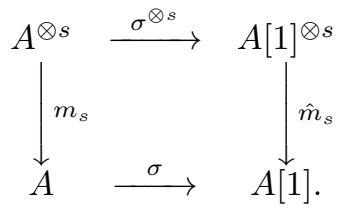

Here, if $r=0$ (or $t=0$ ) then $i d^{\otimes r}$ (resp. $i d^{\otimes t}$ ) is omitted.

Then, for $n \geq 1$, making $\Delta_{n}:=\widehat{\delta}(\hat{f})_{n}\left(\sigma_{M} \otimes \sigma^{\otimes n}\right)$ we have

$$
\begin{aligned}
& \Delta_{n}=\sum_{r+s+t=n}(-1)^{|\hat{f}|+1} \hat{f}\left(i d_{M[1]} \otimes i d^{\otimes r} \otimes \hat{m}_{s} \otimes i d^{\otimes t}\right)\left(\sigma_{M} \otimes \sigma^{\otimes n}\right) \\
& =\sum_{r+s+t=n}(-1)^{|f|+r} \hat{f}_{n-s+1}\left(\sigma_{M} \otimes \sigma^{\otimes r} \otimes \hat{m}_{s} \sigma^{\otimes s} \otimes \sigma^{\otimes t}\right) \\
& r, t \geq 0 ; s \geq 1 \\
& =\sum_{r+s+t=n}(-1)^{|f|+r} \hat{f}_{n-s+1}\left(\sigma_{M} \otimes \sigma^{\otimes r} \otimes \sigma m_{s} \otimes \sigma^{\otimes t}\right) \\
& =\sum_{r+s+t=n}^{r, t \geq 0 ; s \geq 1}(-1)^{|f|+r+s t} \hat{f}_{n-s+1}\left(\sigma_{M} \otimes \sigma^{\otimes n}\right)\left(i d_{M} \otimes i d^{\otimes r} \otimes m_{s} \otimes i d^{\otimes t}\right) \\
& =\quad \begin{array}{c}
r, t \geq 0 ; s \geq 1 \\
r+s+t=n
\end{array}(-1)^{|f|+r+s t} \sigma_{N} f_{n-s+2}\left(i d_{M} \otimes i d^{\otimes r} \otimes m_{s} \otimes i d^{\otimes t}\right) \\
& =\sum_{r+s+t=n+1}^{r, t \geq 0 ; s \geq 1}(-1)^{|f|+r+s t+1} \sigma_{N} f_{(n+1)-s+1}\left(i d^{\otimes r} \otimes m_{s} \otimes i d^{\otimes t}\right) \\
& t \geq 0 ; r, s \geq 1 \\
& =\sigma_{N} \delta_{\infty}(f)_{n+1} \text {. }
\end{aligned}
$$


Finally, we have

$$
\begin{aligned}
\widehat{\delta}(\hat{f})_{0} \zeta \sigma_{M} & =\widehat{\delta}(\hat{f})_{0}\left(\sigma_{M} \otimes 1\right) \\
& =(-1)^{|\hat{f}|+1} \hat{f}\left(i d_{M[1]} \otimes \delta\right)\left(\sigma_{M} \otimes 1\right) \\
& =0=\sigma_{N} \delta_{\infty}(f)_{1} .
\end{aligned}
$$

This finishes the proof.

Definition 9.3. Given an $A_{\infty}$-algebra $A=\left(A,\left\{m_{n}\right\}\right)$, we will denote by TGMod- $A$ the following graded $k$-category. Its objects $\left(M, m^{M}\right)$ are graded right $S$-modules $M$ equipped with a morphism $m^{M}: M \longrightarrow M$ in GMod- $A$ with degree $\left|m^{M}\right|=1$ such that the following Maurer Cartan equation holds

$$
\delta_{\infty}\left(m^{M}\right)+m^{M} \circ m^{M}=0 .
$$

A homogeneous morphism $f:\left(M, m^{M}\right) \longrightarrow\left(N, m^{N}\right)$ in TGMod-A with degree $|f|=d$ is a homogeneous morphism $f: M \longrightarrow N$ is GMod- $A$ with degree $d$ such that the following equation holds

$$
\delta_{\infty}(f)+m^{N} \circ f-(-1)^{|f|} f \circ m^{M}=0 .
$$

The composition in TGMod- $A$ is the same composition of GMod- $A$.

Again, the fact that the preceding notions give rise indeed to a graded $k$ category follows from the following statement, which is quite clear.

Proposition 9.4. Let $\mathcal{B}_{A}$ denote the differential tensor $S$-bocs associated to the $A_{\infty}$-algebra $A$, then there is an equivalence of graded $k$-categories

$$
\mathbb{G}: \text { TGMod- } A \longrightarrow \text { TGMod- } \mathcal{B}_{A} \text {. }
$$

Given $\left(M, m^{M}\right) \in$ GMod- $A$, by definition $\mathbb{G}\left(M, m^{M}\right)=\left(M[1], \mathbb{G}\left(m^{M}\right)\right)$.

Given a homogeneous morphism $f:\left(M, m^{M}\right) \longrightarrow\left(N, m^{N}\right)$ in TGMod- $A$ with degree $d$, we look at the underlying morphism $f: M \longrightarrow N$ in GMod- $A$ and apply $\mathbb{G}$ to obtain a morphism $\mathbb{G}(f): \mathbb{G}(M) \longrightarrow \mathbb{G}(N)$ in GMod- $\mathcal{B}_{A}$, such that $\mathbb{G}(f): \mathbb{G}\left(M, m^{M}\right) \longrightarrow \mathbb{G}\left(N, m^{N}\right)$ is a morphism in TGMod- $\mathcal{B}_{A}$.

Proposition 9.5. Let $\mathcal{B}_{A}$ denote the differential tensor $S$-bocs associated to an $A_{\infty}$-algebra $A$. Then, we have:

1. The preceding requirements for a pair $\left(M, m^{M}\right) \in \mathrm{TGMod}-A$ mean that $\left(M, m^{M}\right)$ is a right $A_{\infty}$-module over $A$ in the usual sense of (1.3).

2. The preceding requirements for a family of morphisms $f=\left\{f_{n}\right\}_{n \in \mathbb{N}}$ to be a homogeneous morphism of degree $d$ from $\left(M, m^{M}\right)$ to $\left(N, m^{N}\right)$ in TGMod- $A$ translate into the following. They mean that $f$ is a family $f=\left\{f_{n}\right\}_{n \in \mathbb{N}}$, where each

$$
f_{n}: M \otimes_{S} A^{\otimes(n-1)} \longrightarrow N
$$


is a homogeneous morphism of graded right $S$-modules of degree $\left|f_{n}\right|=$ $d+1-n$ such that, for each $n \in \mathbb{N}$, the equality $\Sigma_{n}^{f+}+\Sigma_{n}^{f 0}+\Sigma_{n}^{f-}=0$ holds, where

$$
\begin{gathered}
\Sigma_{n}^{f+}=\sum_{\substack{r+s+t=n \\
t \geq 0 ; r, s \geq 1}}(-1)^{|f|+r+s t} f_{r+1+t}\left(i d^{\otimes r} \otimes m_{s} \otimes i d^{\otimes t}\right), \\
\Sigma_{n}^{f 0}=\sum_{\substack{s+t=n \\
t \geq 0 ; s \geq 1}}(-1)^{|f|+s t} f_{1+t}\left(m_{s}^{M} \otimes i d^{\otimes t}\right),
\end{gathered}
$$

and

$$
\Sigma_{n}^{f-}=\sum_{\substack{r+s=n \\ r \geq 1 ; s \geq 0}}(-1)^{(|f|+r+1) s+1} m_{1+s}^{N}\left(f_{r} \otimes i d^{\otimes s}\right) .
$$

The condition in case $n=1$ is equivalent to $m_{1}^{N} f_{1}=f_{1} m_{1}^{M}$, that is, to the requirement that the map $f_{1}: M \longrightarrow N$ is a morphism of complexes of right $S$-modules.

Then, if we restrict to the case where $f$ has degree $|f|=0$, we have that $f:\left(M, m^{M}\right) \longrightarrow\left(N, m^{N}\right)$ is a morphism of right $A_{\infty}$-modules in the usual sense of (1.3).

Therefore, the usual category $\operatorname{Mod}_{\infty}-A$ of right $A_{\infty}$-modules over the $A_{\infty}$-algebra $A$ is the subcategory $\mathrm{TGMod}^{0}-A$ of TGMod- $A$ with the same objects and the homogeneous morphisms of degree 0 between them. Then, we can restrict the functor $\mathbb{G}$ to an equivalence functor $\mathbb{G}: \operatorname{Mod}_{\infty}-A \longrightarrow \mathrm{TGMod}^{0}-\mathcal{B}_{A}$.

Proof. Item (1) follows from the observation that, for $n \geq 1$, we have

$$
\delta_{\infty}\left(m^{M}\right)_{n}=\Sigma_{n}^{+} \text {and }\left(m^{M} \circ m^{M}\right)_{n}=\Sigma_{n}^{0} .
$$

Item (2) follows from the observation that, for $n \geq 1$, we have

$$
\delta_{\infty}(f)_{n}=-\sum_{n}^{f+}, \quad\left(m^{N} \circ f\right)_{n}=-\sum_{n}^{f-}, \text { and }(-1)^{|f|}\left(f \circ m^{M}\right)_{n}=\sum_{n}^{f 0} .
$$

Proposition 9.6. Let $A$ be an $A_{\infty}$-algebra. Then, the category $\mathrm{GMod}^{0}-A$ is additive with split idempotents. Denote by $\mathcal{E}_{0}$ the class of composable morphisms $M \stackrel{f}{\longrightarrow} E \stackrel{g}{\longrightarrow} N$ in $\mathrm{GMod}^{0}-A$ such that $g \circ f=0$ and

$$
0 \longrightarrow M \stackrel{f_{1}}{\longrightarrow} E \stackrel{g_{1}}{\longrightarrow} N \longrightarrow 0
$$

is an exact sequence in GMod-S. Then we have:

1. The pair $\left(\operatorname{GMod}^{0}-A, \mathcal{E}_{0}\right)$ is an exact category. The class $\mathcal{E}_{0}$ consists of the split exact pairs in $\mathrm{GMod}^{0}-A$. 
2. A morphism $f: M \longrightarrow E$ in $\operatorname{GMod}^{0}-A$ is an $\mathcal{E}_{0}$-inflation iff $f_{1}: M \longrightarrow E$ is injective.

3. A morphism $g: E \longrightarrow N$ in $\mathrm{GMod}^{0}-A$ is an $\mathcal{E}_{0}$-deflation iff $g_{1}: E \longrightarrow N$ is surjective.

Proof. Recall that the coalgebra $\mathcal{B}_{A}=\left(T_{S}(A[1]), \mu, \epsilon, \delta\right)$ associated by the bar construction to the $A_{\infty}$-algebra $A$ is a triangular differential graded $S$-bocs. Then, we have (6.7) and (6.11), and we can translate them to $\mathrm{GMod}^{0}-A$ with the equivalence $\mathbb{G}: \mathrm{GMod}^{0}-A \longrightarrow \mathrm{GMod}^{0}-\mathcal{B}_{A}$.

Proposition 9.7. Let $A$ be an $A_{\infty}$-algebra. Then, the category $\operatorname{Mod}_{\infty}-A$ is additive with split idempotents. Denote by $\mathcal{E}_{\infty}$ the class of composable morphisms in $\operatorname{Mod}_{\infty}-A$

$$
\left(M, m^{M}\right) \stackrel{f}{\longrightarrow}\left(E, m^{E}\right) \stackrel{g}{\longrightarrow}\left(N, m^{N}\right)
$$

such that $g \circ f=0$ and the short sequence of first components

$$
0 \longrightarrow M \stackrel{f_{1}}{\longrightarrow} E \stackrel{g_{1}}{\longrightarrow} N \longrightarrow 0
$$

is an exact sequence in GMod-S. Then we have the following.

1. The pair $\left(\operatorname{Mod}_{\infty}-A, \mathcal{E}_{\infty}\right)$ is an exact category. The class $\mathcal{E}_{\infty}$ coincides with the class of composable morphisms such that when we forget the second components of the objects, we have a split exact pair in $\operatorname{GMod}^{0}-A$.

2. A morphism $f:\left(M, m^{M}\right) \longrightarrow\left(E, m^{E}\right)$ in $\operatorname{Mod}_{\infty}-A$ is an $\mathcal{E}_{\infty}$-inflation iff $f_{1}: M \longrightarrow E$ is injective.

3. A morphism $g:\left(E, m^{E}\right) \longrightarrow\left(N, m^{N}\right)$ in $\operatorname{Mod}_{\infty}-A$ is an $\mathcal{E}_{\infty}$-deflation iff $g_{1}: E \longrightarrow N$ is surjective.

4. The functor $\mathbb{G}:\left(\operatorname{Mod}_{\infty}-A, \mathcal{E}_{\infty}\right) \longrightarrow\left(\operatorname{TGMod}^{0}-\mathcal{B}_{A}, \mathcal{E}\right)$ is an equivalence of exact categories.

Proof. As before, the $S$-coalgebra $\mathcal{B}_{A}$ associated by the bar construction to the $A_{\infty}$-algebra $A$ is a triangular differential graded $S$-bocs. Then, we can apply (6.7) to $\mathrm{TGMod}^{0}-\mathcal{B}_{A}$. Moreover, we can translate (7.5) to $\operatorname{Mod}_{\infty}-A=$ TGMod ${ }^{0}-A$ with the equivalence functor $\mathbb{G}: \operatorname{Mod}_{\infty}-A \longrightarrow \operatorname{TGMod}^{0}-\mathcal{B}_{A}$.

Remark 9.8. Given an $A_{\infty}$-algebra $A$, as we did in (7.7), we can associate to any given $M \in$ GMod- $A$ the homogeneous isomorphism $\underline{\sigma}_{M}: M \longrightarrow M[1]$ in GMod- $A$ of degree -1 such that $\underline{\sigma}_{M}=\left\{\sigma_{n}\right\}_{n \in \mathbb{N}}$, where $\sigma_{1}:=\sigma_{M}: M \longrightarrow M[1]$ and, for $n \geq 2, \sigma_{n}:=0: M \otimes A^{\otimes(n-1)} \longrightarrow M[1]$.

Lemma 9.9. Let $A$ be an $A_{\infty}$-algebra. Then, we have:

1. For each $M \in$ GMod- $A$, make $T(M)=M[1]$ and, for any morphism $f: M \longrightarrow N$ in GMod- $A$, make

$$
T(f)=f[1]=\underline{\sigma}_{N} \circ f \circ \underline{\sigma}_{M}^{-1}: M[1] \longrightarrow N[1] \text { in GMod }-A .
$$


Then, we have an autofunctor $T:$ GMod- $A \longrightarrow$ GMod- $A$ with inverse $T^{-1}$ given by $T^{-1}(M)=M[-1]$ and

$$
T^{-1}(f)=f[-1]=\underline{\sigma}_{N[-1]}^{-1} \circ f \circ \underline{\sigma}_{M[-1]}: M[-1] \longrightarrow N[-1] \text { in GMod- } A .
$$

It is called the shifting autofunctor of GMod-A.

2. For each $\left(M, m^{M}\right) \in$ TGMod- $A$, make $T\left(M, m^{M}\right)=\left(M[1],-m^{M}[1]\right)$ and, for any morphism $f:\left(M, m^{M}\right) \longrightarrow\left(N, m^{N}\right)$ in GMod- $A$, make

$T(f)=f[1]=\underline{\sigma}_{N} \circ f \circ \underline{\sigma}_{M}^{-1}:\left(M[1],-m^{M}[1]\right) \longrightarrow\left(N[1],-m^{N}[1]\right)$ in TGMod- $A$.

Then, we have an autofunctor $T:$ TGMod- $A \longrightarrow$ TGMod- $A$ with inverse $T^{-1}$ given by $T^{-1}\left(M, m^{M}\right)=\left(M[-1],-m^{M}[-1]\right)$ and

$T^{-1}(f)=f[-1]=\underline{\sigma}_{N[-1]}^{-1} \circ f \circ \underline{\sigma}_{M[-1]}:\left(M[-1],-m^{M}[-1]\right) \longrightarrow\left(N[-1],-m^{N}[-1]\right)$.

It is called the shifting autofunctor of TGMod-A.

3. The functor $\mathbb{G}:$ GMod- $A \longrightarrow$ GMod- $\mathcal{B}_{A}$ commutes with the corresponding shifting autofunctors, and the functor $\mathbb{G}:$ TGMod- $A \longrightarrow$ TGMod- $\mathcal{B}_{A}$ commutes with the corresponding shifting autofunctors.

4. Furthermore, the shifting functor $T:\left(\operatorname{Mod}_{\infty}-A, \mathcal{E}_{\infty}\right) \longrightarrow\left(\operatorname{Mod}_{\infty}-A, \mathcal{E}_{\infty}\right)$ is an automorphism of exact categories. As a consequence, $T$ and $T^{-1}$ preserve the classes of $\mathcal{E}_{\infty}$-injectives and $\mathcal{E}_{\infty}$-projectives.

Proof. It follows from (7.8).

Proposition 9.10. For $\left(M, m^{M}\right) \in$ TGMod- $A$, we write $m^{M[1]}:=-m^{M}[1]$, so we have $T\left(M, m^{M}\right)=\left(M[1], m^{M[1]}\right)$. There is an endofunctor

$$
J: \text { TGMod- } A \longrightarrow \text { TGMod- } A
$$

such that, for any $\left(M, m^{M}\right) \in$ TGMod-A we have

$$
J\left(M, m^{M}\right)=\left(M \oplus M[1],\left(\begin{array}{cc}
m^{M} & \underline{\sigma}_{M}^{-1} \\
0 & m^{M[1]}
\end{array}\right)\right)
$$

and, given a morphism $f:\left(M, m^{M}\right) \longrightarrow\left(N, m^{N}\right)$ in TGMod- $A$, the morphism $J(f)$ is given by the matrix

$$
J(f)=\left(\begin{array}{cc}
f & 0 \\
0 & f[1]
\end{array}\right): J\left(M, m^{M}\right) \longrightarrow J\left(N, m^{N}\right) .
$$

The equivalence $\mathbb{G}:$ TGMod- $A \longrightarrow$ TGMod- $\mathcal{B}_{A}$ satisfies $\mathbb{G} J=J \mathbb{G}$.

Moreover, there are natural transformations $\alpha: i d_{\operatorname{Mod}_{\infty}-A} \longrightarrow J$ and $\beta$ : $J \longrightarrow T$ of endofunctors of $\operatorname{Mod}_{\infty}-A$ such that, for each $\underline{M}=\left(M, m^{M}\right) \in$ $\operatorname{Mod}_{\infty}-A$, we have an $\mathcal{E}_{\infty}$-conflation

$$
\underline{M} \stackrel{\alpha_{\underline{M}}}{\longrightarrow} J(\underline{M}) \stackrel{\beta_{\underline{N}}}{\longrightarrow} T(\underline{M}) .
$$


For $\underline{M} \in \operatorname{Mod}_{\infty}-A$, the morphisms $\alpha_{\underline{M}}$ and $\beta_{\underline{M}}$ are strict morphisms with

$$
\left(\alpha_{\underline{M}}\right)_{1}=\left(i d_{M}, 0\right)^{t}: M \longrightarrow M \oplus M[1]
$$

and

$$
\left(\beta_{\underline{M}}\right)_{1}=\left(0, i d_{M[1]}\right): M \oplus M[1] \longrightarrow M[1] .
$$

Then, the category $\operatorname{Mod}_{\infty}-A$ has enough $\mathcal{E}_{\infty}$-projectives and enough $\mathcal{E}_{\infty}$-injectives and, moreover, the class of $\mathcal{E}_{\infty}$-projectives coincides with the class of $\mathcal{E}_{\infty}$-injectives. So, we obtain that $\operatorname{Mod}_{\infty}-A$ is a special Frobenius category.

Proof. It follows from (7.9), and (7.12), which are translated into $\operatorname{Mod}_{\infty}-A$ with the help of the functor $\mathbb{G}$. Then, we use (9.7) and (9.9).

Lemma 9.11. Let $A$ be an $A_{\infty}$-algebra. Then, we have the following explicit descriptions for the shifting functor $T$ and the functor $J$.

1. Given $\left(M, m^{M}\right) \in$ TGMod- $A$, its translate $\left(M, m^{M}\right)[1]=\left(M[1], m^{M[1]}\right)$ is given for each $n \in \mathbb{N}$, by

$$
m_{n}^{M[1]}=(-1)^{n} \sigma_{M} m_{n}^{M}\left(\sigma_{M}^{-1} \otimes i d^{\otimes(n-1)}\right) .
$$

2. Given a morphism $f:\left(M, m^{M}\right) \longrightarrow\left(N, m^{N}\right)$ in TGMod- $A$, its translate $f[1]:\left(M[1], m^{M[1]}\right) \longrightarrow\left(N[1], m^{N[1]}\right)$ is given, for each $n \in \mathbb{N}$, by

$$
f[1]_{n}=(-1)^{n-1} \sigma_{N} f_{n}\left(\sigma_{M}^{-1} \otimes i d^{\otimes(n-1)}\right) .
$$

3. Given $\left(M, m^{M}\right) \in$ TGMod- $A$, we have $J\left(M, m^{M}\right)=\left(M \oplus M[1], m^{J(M)}\right)$, where

$$
m_{n}^{J(M)}: M \otimes A^{\otimes(n-1)} \oplus M[1] \otimes A^{\otimes(n-1)} \longrightarrow M \oplus M[1]
$$

with

$$
m_{1}^{J(M)}=\left(\begin{array}{cc}
m_{1}^{M} & \sigma_{M}^{-1} \\
0 & -m_{1}^{M}[1]
\end{array}\right) \text { and } m_{n}^{J(M)}=\left(\begin{array}{cc}
m_{n}^{M} & 0 \\
0 & -m_{n}^{M}[1]
\end{array}\right) \text { for } n \geq 2 \text {. }
$$

4. Given a morphism $f:\left(M, m^{M}\right) \longrightarrow\left(N, m^{N}\right)$ in TGMod-A, its translate $J(f):\left(M \oplus M[1], m^{J(M)}\right) \longrightarrow\left(N \oplus N[1], m^{J(N)}\right)$ is given, for each $n \in \mathbb{N}$, by

$$
J(f)_{n}: M \otimes A^{\otimes(n-1)} \oplus M[1] \otimes A^{\otimes(n-1)} \longrightarrow M \oplus M[1]
$$

with matrix

$$
J(f)_{n}=\left(\begin{array}{cc}
f_{n} & 0 \\
0 & (-1)^{n-1} \sigma_{N} f_{n}\left(\sigma_{M}^{-1} \otimes i d^{(n-1)}\right)
\end{array}\right) .
$$


Proof. (1) and (2): It will be enough to show that for any morphism $f: M \longrightarrow N$ in GMod- $A$, its translate $f[1]: M[1] \longrightarrow N[1]$ in GMod- $A$ is given, for each $n \in \mathbb{N}$, by

$$
f[1]_{n}=(-1)^{n-1} \sigma_{N} f_{n}\left(\sigma_{M}^{-1} \otimes i d^{\otimes(n-1)}\right) .
$$

Indeed, from the definition of $f[1]$ and of the composition in GMod- $A$, we have

$$
\begin{aligned}
& f[1]_{n}=\left(\underline{\sigma}_{N} \circ f \circ \underline{\sigma}_{M}^{-1}\right)_{n} \\
& =\sum_{\substack{r+s=n \\
r \geq 1 ; s \geq 0}}(-1)^{\left(\left|f \circ \underline{\sigma}_{M}^{-1}\right|+r+1\right) s}\left(\underline{\sigma}_{N}\right)_{1+s}\left(\left(f \circ \underline{\sigma}_{M}^{-1}\right)_{r} \otimes i d^{\otimes s}\right) \\
& =\sigma_{N}\left(f \circ \underline{\sigma}_{M}^{-1}\right)_{n} \\
& =\sigma_{N}\left(\sum_{r+s=n}(-1)^{\left(\left|\underline{\sigma}_{M}^{-1}\right|+r+1\right) s} f_{1+s}\left(\left(\underline{\sigma}_{M}^{-1}\right)_{r} \otimes i d^{\otimes s}\right)\right. \\
& =(-1)^{n-1} \sigma_{N} f_{n}\left(\sigma_{M}^{-1} \otimes i d^{\otimes(n-1)}\right) .
\end{aligned}
$$

Items (3) and (4) clearly follow from (1) and (2).

Remark 9.12. Let $A$ be an $A_{\infty}$-algebra and consider a pair of morphisms $f, g \in$ $\operatorname{Hom}_{\operatorname{Mod}_{\infty}-A}\left(\left(M, m^{M}\right),\left(N, m^{N}\right)\right)$. Then, there is a homotopy $h=\left\{h_{n}\right\}_{n \in \mathbb{N}}$ from $f$ to $g$, as in definition (1.4), iff there is a morphism $h \in \operatorname{Hom}_{\mathrm{GMod}-A}^{-1}(M, N)$ satisfying

$$
f-g=\delta_{\infty}(h)+m^{N} \circ h+h \circ m^{M} .
$$

Indeed, the preceding formula translates into the usual formulas given in (1.4): For $n \geq 1$, we have $\delta_{\infty}(h)_{n}=H_{n}^{(3)},\left(m^{N} \circ h\right)_{n}=H_{n}^{(1)}$, and $\left(h \circ m^{M}\right)_{n}=H_{n}^{(2)}$.

Proposition 9.13. Let $A$ be an $A_{\infty}$-algebra. Then, the functor

$$
\mathbb{G}: \operatorname{Mod}_{\infty}-A \longrightarrow \mathrm{TGMod}^{0}-\mathcal{B}_{A}
$$

preserves and reflects homotopies. Therefore, it induces an equivalence of these categories modulo homotopy

$$
\mathbb{G}: \underline{\operatorname{Mod}}_{\infty}-A \longrightarrow{\underline{\mathrm{TGMod}^{0}}}^{-} \mathcal{B}_{A}
$$

Definition 9.14. Let $A$ be an $A_{\infty}$-algebra. Then, a sequence of morphisms

$$
\left(M, m^{M}\right) \stackrel{\underline{f}}{\longrightarrow}\left(E, m^{E}\right) \stackrel{\underline{g}}{\longrightarrow}\left(N, m^{N}\right) \stackrel{\underline{h}}{\longrightarrow} T\left(M, m^{M}\right)
$$

in the homotopy category $\underline{\operatorname{Mod}}_{\infty}-A$ such that

$$
\xi:\left(M, m^{M}\right) \stackrel{f}{\longrightarrow}\left(E, m^{E}\right) \stackrel{g}{\longrightarrow}\left(N, m^{N}\right)
$$

is an exact pair in $\mathcal{E}_{\infty}$ and we have a commutative diagram of the form

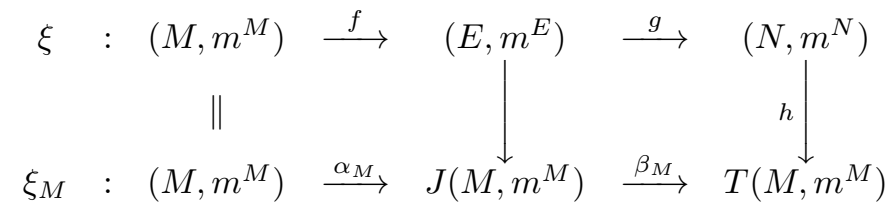


in $\operatorname{Mod}_{\infty}-A$ is called a canonical triangle in $\underline{\operatorname{Mod}}_{\infty}-A$. Now, consider the class $\mathcal{T}_{\infty}$ of sequences of morphisms

$$
X \stackrel{u}{\longrightarrow} Y \stackrel{v}{\longrightarrow} Z \stackrel{w}{\longrightarrow} T X
$$

in $\underline{\operatorname{Mod}}_{\infty}-A$ which are isomorphic to canonical triangles. That is, there is a canonical triangle $M \stackrel{f}{\longrightarrow} E \stackrel{\underline{g}}{\longrightarrow} N \stackrel{\underline{h}}{\longrightarrow} T M$ and isomorphisms $a, b, c$ such that the following diagram commutes

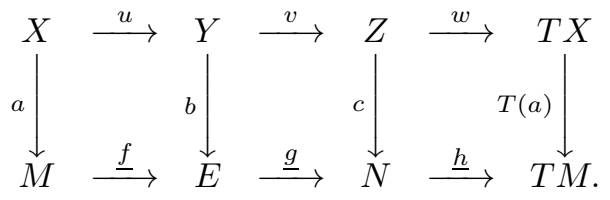

The elements of $\mathcal{T}_{\infty}$ are called the triangles of $\underline{\operatorname{Mod}}_{\infty}-A$.

As in (7.16), the next statement follows from the general result for special Frobenius categories.

Theorem 9.15. Let $A$ be an $A_{\infty}$-algebra and consider its associated differential tensor $S$-bocs $\mathcal{B}_{A}$. The stable category $\underline{\operatorname{Mod}}_{\infty}-A$ with the automorphism $T$ and the class of triangles $\mathcal{T}_{\infty}$ defined above is a triangulated category. The functor $\mathbb{G}: \operatorname{Mod}_{\infty}-A \longrightarrow \operatorname{TGMod}^{0}-\mathcal{B}_{A}$ induces an equivalence of triangulated categories

$$
\underline{\mathbb{G}}: \underline{\operatorname{Mod}}_{\infty}-A \longrightarrow \underline{\mathrm{TGMod}}^{0}-\mathcal{B}_{A} \text {. }
$$

From the preceding theorem and (8.4), we immediately obtain the following important statement, see [6](5.2).

Theorem 9.16. Assume that $S$ is a finite product of copies of the field $k$ and let $A$ be an $A_{\infty}$-algebra. Then, every quasi-isomorphism of $A_{\infty}$-modules is a homotopy equivalence, hence an invertible morphism in $\underline{\operatorname{Mod}}_{\infty}-A$.

\section{Restriction functors for $A_{\infty}$-modules}

In this section we show how the result (5.5) on restriction functors for triangular differential graded $S$-bocses translates into the corresponding result for restriction functors for $A_{\infty}$-algebras. We start by recalling the following link between homotopies in $\mathrm{Alg}_{\infty}$ and DGCoalg, as defined in (1.5) and (5.1).

Lemma 10.1. The functor $\Psi: \mathrm{Alg}_{\infty} \longrightarrow$ DGCoalg preserves and reflects nullhomotopic morphisms.

For the sake of completeness, we notice that this follows, for instance, from the following statement.

Lemma 10.2. Given morphisms of $A_{\infty}$-algebras $f, g: A \longrightarrow B$ and $d \in \mathbb{Z}$, we consider the set $\mathcal{H}_{\infty}^{d}(A, B)$ formed by the families $h=\left\{h_{n}\right\}_{n \in \mathbb{N}}$, such that, for each $n \in \mathbb{N}$, the map $h_{n}: A^{\otimes n} \longrightarrow B$ is a homogeneous morphism of $S$-Sbimodules of degree $\left|h_{n}\right|=d+1-n$. Make $\phi:=\Psi(f)$ and $\psi=\Psi(g)$. Then, 
1. We have a linear isomorphism

$$
\Delta=\Delta_{f, g}: \mathcal{H}_{\infty}^{d}(A, B) \longrightarrow \operatorname{Coder}_{\phi, \psi}^{d}\left(\mathcal{B}_{A}, \mathcal{B}_{B}\right)
$$

constructed as follows. Given $h \in \mathcal{H}_{\infty}^{d}(A, B)$, we consider the morphism of $S$-S-bimodules $\widehat{h}: \bar{T}_{S}(A[1]) \longrightarrow B[1]$ of degree $|\widehat{h}|=d$ determined by the commutativity of the squares

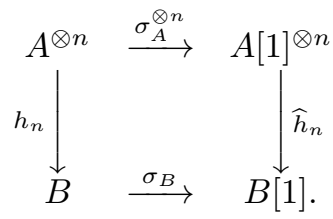

Then, consider the $\bar{\Psi}(f)-\bar{\Psi}(g)$-coderivation $\bar{\Delta}(\widehat{h}): \bar{T}_{S}(A[1]) \longrightarrow \bar{T}_{S}(B[1])$ given by $\widehat{h}$ and the universal property of the reduced tensor $S$-coalgebra $\bar{T}_{S}(A[1])$. Finally, extend $\bar{\Delta}(\widehat{h})$ to a $\Psi(f)-\Psi(g)$-coderivation of degree $d$

$$
\Delta(h): T_{S}(A[1]) \longrightarrow T_{S}(B[1]) .
$$

2. If $h \in \mathcal{H}_{\infty}^{-1}(A, B)$, with the notation of (1.5), we have

$$
h^{\odot}:=H(h)-H_{f, g}(h) \in \mathcal{H}_{\infty}^{0}(A, B) .
$$

3. We have $\Psi(f)-\Psi(g) \in \operatorname{Coder}_{\phi, \psi}^{0}\left(\mathcal{B}_{A}, \mathcal{B}_{B}\right)$.

4. For any $\phi-\psi$-coderivation $\xi \in \operatorname{Coder}_{\phi, \psi}^{-1}\left(\mathcal{B}_{A}, \mathcal{B}_{B}\right)$, we have

$$
\xi^{\odot}:=\delta_{B} \xi+\xi \delta_{A} \in \operatorname{Coder}_{\phi, \psi}^{0}\left(\mathcal{B}_{A}, \mathcal{B}_{B}\right)
$$

5. For any $h \in \mathcal{H}_{\infty}^{-1}(A, B)$, we have $\Delta\left(h^{\odot}\right)=\Delta(h)^{\odot}$.

6. We have $\Delta(f-g)=\phi-\psi$.

7. The map $\Delta$ induces a bijection between the homotopy sets $\mathcal{H}(f, g)$ and $\mathcal{H}(\phi, \psi)$.

As a consequence, the morphisms $f$ and $g$ are homotopic in $\mathrm{Alg}_{\infty}$ if and only if the morphisms $\phi$ and $\psi$ are homotopic in DGCoalg.

Proof. The map $\mathcal{H}_{\infty}^{d}(A, B) \longrightarrow \operatorname{Hom}_{\text {GMod-S-S }}^{d}\left(\bar{T}_{S}(A[1]), B[1]\right)$ is clearly an isomorphism, and so is the map

$$
\bar{\Delta}: \operatorname{Hom}_{\mathrm{GMod}-S-S}^{d}\left(\bar{T}_{S}(A[1]), B[1]\right) \longrightarrow \operatorname{Coder}_{\bar{\Psi}(f), \bar{\Psi}(g)}^{d}\left(\bar{T}_{S}(A[1]), \bar{T}_{S}(B[1])\right)
$$

given by the universal property of the reduced tensor $S$-coalgebra. Recall that the inverse of $\bar{\Delta}$ maps each coderivation $\xi$ onto $p_{B} \xi$, where $p_{B}: \bar{T}_{S}(B[1]) \longrightarrow B[1]$ is the projection. Finally, the extension $\bar{\Delta}(\widehat{h}) \mapsto \Delta(h)$ is also bijective. 
(2)-(4) are easy to show. In order to show (5), take $h \in \mathcal{H}_{\infty}^{-1}(A, B)$. Make $\bar{\Delta}(\widehat{h})^{\odot}:=\bar{\delta}_{B} \bar{\Delta}(\hat{h})+\bar{\Delta}(\hat{h}) \bar{\delta}_{A}$. Notice that it will be enough to show the equality $\bar{\Delta}\left(\widehat{h^{\odot}}\right)=\bar{\Delta}(\widehat{h})^{\odot}$. Since both terms are $\phi$ - $\psi$-coderivations, it will be enough to show that $p_{B} \bar{\Delta}\left(\widehat{h^{\odot}}\right)_{n}=p_{B} \bar{\Delta}(\widehat{h})_{n}^{\odot}$, for all $n \geq 1$. This means that

$$
(\widehat{h \odot})_{n}=p_{B}\left(\bar{\delta}_{B} \bar{\Delta}(\widehat{h})+\bar{\Delta}(\widehat{h}) \bar{\delta}_{A}\right)_{n}, \text { for all } n \geq 1 .
$$

We have $p_{B}\left(\bar{\delta}_{B} \bar{\Delta}(\hat{h})+\bar{\Delta}(\hat{h}) \bar{\delta}_{A}\right)_{n}=D_{n}+R_{n}$ where

$$
D_{n}=p_{B} \bar{\delta}_{B} \bar{\Delta}(\hat{h})_{n} \text { and } R_{n}=p_{B} \bar{\Delta}(\hat{h})\left(\bar{\delta}_{A}\right)_{n} .
$$

From explicit description of $\bar{\Delta}(\widehat{h})$, we have

$$
\begin{aligned}
D_{n}= & \sum \begin{array}{c}
r, t \geq 0 ; s \geq 1 \\
i_{1}+\cdots+i_{r}+s \\
+j_{1}+\cdots+j_{t}=n
\end{array} \\
= & p_{B} \bar{\delta}_{B}\left(\hat{f}_{i_{1}} \otimes \cdots \otimes \hat{f}_{i_{r}} \otimes \hat{h}_{s} \otimes \hat{g}_{j_{1}} \otimes \cdots \otimes \hat{g}_{j_{t}}\right) \\
\begin{array}{c}
r, t \geq 0 ; s \geq 1 \\
i_{1}+\cdots+i_{r}+s \\
+j_{1}+\cdots+j_{t}=n
\end{array} & \widehat{m}_{r+1+t}^{B}\left(\hat{f}_{i_{1}} \otimes \cdots \otimes \hat{f}_{i_{r}} \otimes \hat{h}_{s} \otimes \hat{g}_{j_{1}} \otimes \cdots \otimes \hat{g}_{j_{t}}\right)
\end{aligned}
$$

and

$$
\begin{aligned}
R_{n} & =\sum_{\substack{r+s+t=n \\
r, t \geq 0 ; s \geq 1}} p_{B} \bar{\Delta}(\hat{h})\left(i d^{\otimes r} \otimes \widehat{m}_{s}^{A} \otimes i d^{\otimes t}\right) \\
& =\sum_{\substack{r+s+t=n \\
r, t \geq 0 ; s \geq 1}} \hat{h}_{r+1+t}\left(i d^{\otimes r} \otimes \widehat{m}_{s}^{A} \otimes i d^{\otimes t}\right) .
\end{aligned}
$$

We have

$$
\sigma_{B}^{-1} R_{n} \sigma_{A}^{\otimes n}=\sum_{\substack{r+s+t=n \\ r, t \geq 0 ; s \geq 1}}(-1)^{r+s t} h_{r+1+t}\left(i d^{\otimes r} \otimes m_{s}^{A} \otimes i d^{\otimes t}\right)=H(h)_{n} .
$$

Moreover, we have

$$
\sigma_{B}^{-1} D_{n} \sigma_{A}^{\otimes n}=\sum_{\begin{array}{c}
r, t \geq 0 ; s \geq 1 \\
i_{1}+\cdots+i_{r}+s \\
+j_{1}+\cdots+j_{t}=n
\end{array}} \sigma_{B}^{-1} \widehat{m}_{r+1+t}^{B} Z_{n}\left(i_{1}, \ldots, i_{r}, s, j_{1}, \ldots, j_{t}\right)
$$

where

$$
Z_{n}\left(i_{1}, \ldots, i_{r}, s, j_{1}, \ldots, j_{t}\right)=\left(\hat{f}_{i_{1}} \otimes \cdots \otimes \hat{f}_{i_{r}} \otimes \hat{h}_{s} \otimes \hat{g}_{j_{1}} \otimes \cdots \otimes \hat{g}_{j_{t}}\right) \sigma_{A}^{\otimes n} .
$$

Fix a vector $v=\left(i_{1}, \ldots, i_{r}, s, j_{1}, \ldots, j_{t}\right)$ and let us examine $Z_{n}(v)$. We have $\left(\hat{f}_{i_{1}} \otimes \cdots \otimes \hat{f}_{i_{r}} \otimes \hat{h}_{s} \otimes \hat{g}_{j_{1}} \otimes \cdots \otimes \hat{g}_{j_{t}}\right)\left(\sigma_{A}^{\otimes i_{1}} \otimes \cdots \otimes \sigma_{A}^{\otimes i_{r}} \otimes \sigma_{A}^{\otimes s} \otimes \sigma_{A}^{\otimes j_{1}} \otimes \cdots \otimes \sigma_{A}^{\otimes j_{t}}\right)$ equals

$$
(-1)^{\mathrm{sgn}^{\prime}}\left(\hat{f}_{i_{1}} \sigma_{A}^{\otimes i_{1}} \otimes \cdots \otimes \hat{f}_{i_{r}} \sigma_{A}^{\otimes i_{r}} \otimes \hat{h}_{s} \sigma_{A}^{\otimes k} \otimes \hat{g}_{j_{1}} \sigma_{A}^{\otimes j_{1}} \otimes \cdots \otimes \hat{g}_{j_{t}} \sigma_{A}^{\otimes j_{t}}\right)
$$


and

$$
(-1)^{\mathrm{sgn}^{\prime}}\left(\sigma_{B} f_{i_{1}} \otimes \cdots \otimes \sigma_{B} f_{i_{r}} \otimes \sigma_{B} h_{s} \otimes \sigma_{B} g_{j_{1}} \otimes \cdots \otimes \sigma_{B} g_{j_{t}}\right),
$$

where $\operatorname{sgn}^{\prime}=\sum_{u=1}^{r} i_{u}$. The last term equals

$$
(-1)^{\operatorname{sgn}\left(i_{1}, \ldots, i_{r}, s, j_{1}, \ldots, j_{t}\right)} \sigma_{B}^{\otimes(r+1+t)}\left(f_{i_{1}} \otimes \cdots \otimes f_{i_{r}} \otimes h_{s} \otimes g_{j_{1}} \otimes \cdots \otimes g_{j_{t}}\right),
$$

where the sign was defined in (1.5). Hence, $\sigma_{B}^{-1} D_{n} \sigma_{A}^{\otimes n}=H_{f, g}(h)_{n}$

Therefore, for $n \geq 1$, we get $\sigma_{B}^{-1}\left(D_{n}+R_{n}\right) \sigma_{A}^{\otimes n}=\left(h^{\odot}\right)_{n}$, or, equivalently,

$$
p_{B}\left(\bar{\delta}_{B} \bar{\Delta}(\hat{h})+\bar{\Delta}(\hat{h}) \bar{\delta}_{A}\right)_{n}=\left(\widehat{h^{\odot}}\right)_{n} .
$$

(6): Since $\bar{\Psi}(f)-\bar{\Psi}(g)$ is a $\bar{\Psi}(f)-\bar{\Psi}(g)$-coderivation, from (1), in order to prove that $\bar{\Psi}(f)-\bar{\Psi}(g)=\bar{\Delta}(\widehat{f-g})$, we have to show that

$$
p_{B}(\bar{\Psi}(f)-\bar{\Psi}(g))_{n}=p_{B} \bar{\Delta}(\widehat{f-g})_{n}, \text { for all } n \in \mathbb{N} .
$$

This is clear, because, for $n \in \mathbb{N}$, both terms coincide with $\widehat{f}_{n}-\widehat{g}_{n}$.

(7): If $h$ is a homotopy from $f$ to $g$, then $f-g=h^{\odot}$. Then, we have

$$
\bar{\Psi}(f)-\bar{\Psi}(g)=\bar{\Delta}(\widehat{f-g})=\bar{\Delta}(\widehat{h \odot})=\bar{\Delta}(\widehat{h})^{\odot}=\bar{\delta}_{B} \xi+\xi \bar{\delta}_{A},
$$

where $\xi=\bar{\Delta}(\widehat{h})$ is a homotopy from $\bar{\Psi}(f)$ to $\bar{\Psi}(g)$.

Conversely, if $\xi: \bar{C}_{A} \longrightarrow \bar{C}_{B}$ is a homotopy from $\bar{\Psi}(f)$ to $\bar{\Psi}(g)$, we have $\xi=\bar{\Delta}(\widehat{h})$, for some $h \in \mathcal{H}_{\infty}^{-1}(A, B)$ and

$$
\bar{\Delta}(\widehat{f-g})=\bar{\Psi}(f)-\bar{\Psi}(g)=\bar{\delta}_{A} \xi+\xi \bar{\delta}_{B}=\bar{\Delta}(\widehat{h})^{\odot}=\bar{\Delta}\left(\widehat{h^{\odot}}\right) .
$$

It follows that $f-h=h^{\odot}$, so $h$ is a homotopy from $f$ to $g$.

Proposition 10.3. Let $A$ and $B$ be $A_{\infty}$-algebras and consider a morphism $\phi: A \longrightarrow B$ of $A_{\infty}$-algebras. Then,

1. The morphism $\phi$ induces a restriction functor of differential graded categories

$$
R_{\phi}: \text { GMod- } B \longrightarrow \text { GMod- } A
$$

defined as follows. Given $M \in$ GMod-B, the A-module $R_{\phi}(M)=M$. Given a morphism $f: M \longrightarrow N$ in GMod- $B$, the morphism $R_{\phi}(f)=$ $\left\{R_{\phi}(f)_{n}\right\}_{n \in \mathbb{N}}: R_{\phi}(M) \longrightarrow R_{\phi}(N)$ in GMod- $A$ is given, for $n \geq 2$, by

$$
R_{\phi}(f)_{n}=\sum_{\substack{1 \leq r \leq n-1 \\ i_{1}+\cdots+i_{r}=n-1}}(-1)^{\operatorname{sgn}\left(i_{1}, \ldots, i_{r}\right)} f_{r+1}\left(i d_{M} \otimes \phi_{i_{1}} \otimes \cdots \otimes \phi_{i_{r}}\right),
$$

and $R_{\phi}(f)_{1}=f_{1}$, where $\operatorname{sgn}\left(i_{1}, \ldots, i_{r}\right)$ is as in (1.1). 
2. The morphism $\phi$ induces a restriction functor of graded categories

$$
R_{\phi}: \text { TGMod- } B \longrightarrow \text { TGMod- } A \text {. }
$$

If $\left(M,\left\{m_{n}^{M}\right\}\right) \in$ TGMod-B, by definition, $R_{\phi}\left(M, m^{M}\right)=\left(M, R_{\phi}\left(m^{M}\right)\right)$. Given a morphism $f:\left(M, m^{M}\right) \longrightarrow\left(N, m^{N}\right)$ in TGMod- $B$, the morphism $R_{\phi}(f)=\left\{R_{\phi}(f)_{n}\right\}_{n \in \mathbb{N}}: R_{\phi}(M) \longrightarrow R_{\phi}(N)$ in GMod- $A$ is in fact a morphism $R_{\phi}\left(M, m^{M}\right) \longrightarrow R_{\phi}\left(N, m^{N}\right)$ in TGMod- $A$.

3. Let $\overline{\mathcal{B}}_{A}$ and $\overline{\mathcal{B}}_{B}$ denote the reduced tensor $S$-coalgebras associated to $A$ and $B$ respectively, and denote by $\mathcal{B}_{A}$ and $\mathcal{B}_{B}$ the corresponding tensor $S$-coalgebras. Then the morphism of $A_{\infty}$-algebras $\phi: A \longrightarrow B$ determines a morphism of differential $S$-coalgebras $\bar{\Psi}(\phi): \overline{\mathcal{B}}_{A} \longrightarrow \overline{\mathcal{B}}_{B}$, which extends to a morphism $\Psi(\phi): \mathcal{B}_{A} \longrightarrow \mathcal{B}_{B}$, as in (5.2). Then, we have commutative squares of functors

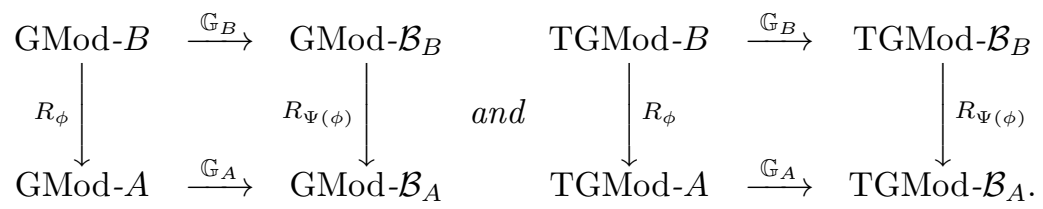

where $\mathbb{G}_{A}$ and $\mathbb{G}_{B}$ are the functors defined in (9.2) and 9.4).

4. Moreover, $R_{\phi}$ maps null-homotopic morphisms of $\operatorname{Mod}_{\infty}-B$ onto nullhomotopic morphisms of $\operatorname{Mod}_{\infty}-A$. So, it induces a functor $\underline{R}_{\phi}$ and a commutative square of functors

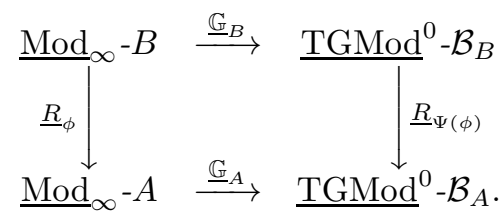

Proof. In order to prove (1), it is enough to show the commutativity of the first diagram in (3), because we already know that $R_{\Psi(\phi)}$ is a functor and $\mathbb{G}_{A}$ and $\mathbb{G}_{B}$ are equivalences, and they all preserve the differentials of the categories. Clearly, $\mathbb{G}_{A} R_{\phi}$ and $R_{\Psi(\phi)} \mathbb{G}_{B}$ coincide on objects. Take a morphism $f: M \longrightarrow N$ in GMod- $B$ and let us show that $\mathbb{G}_{A} R_{\phi}(f)=R_{\Psi(\phi)} \mathbb{G}_{B}(f)$. For this we have to show that, for each $n \geq 0$, we have $\mathbb{G}_{A}\left(R_{\phi}(f)\right)_{n}=R_{\Psi(\phi)} \mathbb{G}_{B}(f)_{n}$. Since $R_{\Psi(\phi)} \mathbb{G}_{B}(f)_{n}=R_{\Psi(\phi)}(\widehat{f})_{n}=\widehat{f}\left(i d_{M[1]} \otimes \Psi(\phi)\right)_{n}$, the former is equivalent to show that ${\widehat{R_{\phi}(f)}}_{n}=\widehat{f}\left(i d_{M[1]} \otimes \Psi(\phi)\right)_{n}$. For this we will show that the following diagrams commute

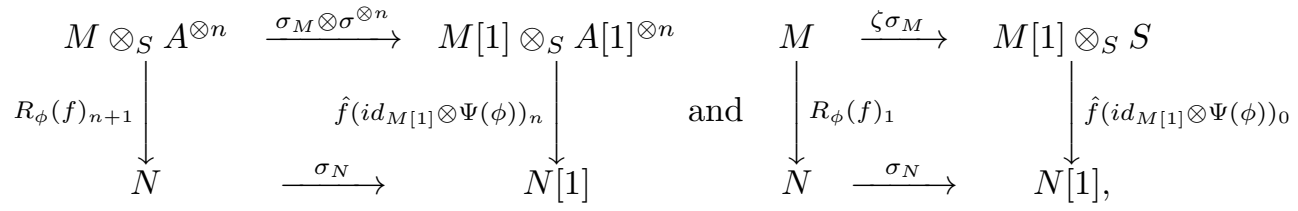


where $n$ runs in $\mathbb{N}$ and $\zeta: M[1] \longrightarrow M[1] \otimes_{S} S$ is the canonical isomorphism.

Recall that, for $n \geq 1$, we have

$$
\Psi(\phi)_{n}=\sum_{\substack{1 \leq r \leq n \\ i_{1}+\cdots+i_{r}=n}} \widehat{\phi}_{i_{1}} \otimes \cdots \otimes \widehat{\phi}_{i_{r}},
$$

where $\widehat{\phi}_{i} \sigma^{\otimes i}=\sigma \phi_{i}$, for all $i \in \mathbb{N}$. Then, if $Q:=\widehat{f}\left(i d_{M[1]} \otimes \Psi(\phi)\right)_{n}\left(\sigma_{M} \otimes \sigma^{\otimes n}\right)$, we have

$$
\begin{aligned}
& Q=\sum_{\substack{1 \leq r \leq n \\
i_{1}+\cdots+i_{r}=n}} \widehat{f}_{r}\left(i d_{M[1]} \otimes \widehat{\phi}_{i_{1}} \otimes \cdots \otimes \widehat{\phi}_{i_{r}}\right)\left(\sigma_{M} \otimes \sigma^{\otimes n}\right) \\
& =\sum_{\substack{1 \leq r \leq n \\
i_{1}+\cdots+i_{r}=n}} \widehat{f}_{r}\left(\sigma_{M} \otimes \widehat{\phi}_{i_{1}} \sigma^{\otimes i_{1}} \otimes \cdots \otimes \widehat{\phi}_{i_{r}} \sigma^{\otimes i_{r}}\right) \\
& =\sum_{\substack{1 \leq r \leq n \\
i_{1}+\cdots+i_{r}=n}} \widehat{f}_{r}\left(\sigma_{M} \otimes \sigma \phi_{i_{1}} \otimes \cdots \otimes \sigma \phi_{i_{r}}\right) \\
& =\sum_{\substack{1 \leq r \leq n \\
i_{1}+\cdots+i_{r}=n}}(-1)^{\mathrm{sgn}} \widehat{f}_{r}\left(\sigma_{M} \otimes \sigma^{\otimes r}\right)\left(i d_{M} \otimes \phi_{i_{1}} \otimes \cdots \otimes \phi_{i_{r}}\right) \\
& =\sum_{\substack{1 \leq r \leq n \\
i_{1}+\cdots+i_{r}=n}}(-1)^{\mathrm{sgn}} \sigma_{N} f_{r+1}\left(i d_{M} \otimes \phi_{i_{1}} \otimes \cdots \otimes \phi_{i_{r}}\right) \\
& =\sigma_{N} R_{\phi}(f)_{n+1} \text {. }
\end{aligned}
$$

For $n=0$, we have

$$
\widehat{f}\left(i d_{M[1]} \otimes \Psi(\phi)\right)_{0} \zeta \sigma_{M}=\widehat{f}_{0}\left(\sigma_{M} \otimes 1\right)=\widehat{f}_{0} \zeta \sigma_{M}=\sigma_{N} f_{1}=\sigma_{N} R_{\phi}(f)_{1} .
$$

(2) and the commutativity of the second square in (3) follow from the commutativity of the first square and the fact that TGMod- $A$ is constructed from GMod- $A$ in a similar way that TGMod- $\mathcal{B}_{A}$ is constructed from GMod- $\mathcal{B}_{A}$, using the corresponding differentials, and the functor $R_{\phi}$ : TGMod- $B \longrightarrow$ TGMod- $A$ is constructed from $R_{\phi}$ : GMod- $B \longrightarrow$ GMod- $A$ in a similar way that the functor $R_{\Psi(\phi)}$ : TGMod- $\mathcal{B}_{B} \longrightarrow$ TGMod- $\mathcal{B}_{A}$ is constructed from the functor $R_{\Psi(\phi)}$ : GMod- $\mathcal{B}_{B} \longrightarrow$ GMod- $\mathcal{B}_{A}$.

(4) Given morphisms $f, g:\left(M, m^{M}\right) \longrightarrow\left(N, m^{N}\right)$ in $\operatorname{Mod}_{\infty}-A$, hence in TGMod $^{0}-A$, any homotopy $h$ from $f$ to $g$ is a homogeneous morphism in GMod- $A$ with degree $|h|=-1$ and is mapped by the restriction functor $R_{\phi}$ : GMod- $B \longrightarrow$ GMod- $A$ onto the homotopy $R_{\psi}(h)$ from $R_{\psi}(f)$ to $R_{\psi}(g)$, see (9.12). Hence, there is an induced functor $\underline{R}_{\phi}$ which clearly makes the diagram of (4) to commute.

Corollary 10.4. Let $f, g: A \longrightarrow B$ be homotopic morphisms of $A_{\infty}$-algebras. Then, there is an isomorphism of functors

$$
\underline{R}_{f} \cong \underline{R}_{g}
$$

where $\underline{R}_{f}, \underline{R}_{g}: \underline{\operatorname{Mod}}_{\infty}-B \longrightarrow \underline{\operatorname{Mod}}_{\infty}-A$ are the functors induced on the homotopy categories by the restriction functors $R_{f}, R_{g}: \operatorname{Mod}_{\infty}-B \longrightarrow \operatorname{Mod}_{\infty}-A$, respectively. Any homotopy equivalence $f: A \longrightarrow B$ of $A_{\infty}$-algebras determines an equivalence of categories $\underline{R}_{f}: \underline{\operatorname{Mod}}_{\infty}-B \longrightarrow \underline{\operatorname{Mod}}_{\infty}-A$. 
Proof. Let $\mathcal{B}_{A}$ and $\mathcal{B}_{B}$ be the differential tensor $S$-coalgebras (or differential tensor $S$-bocses) associated to $A$ and $B$, respectively. Then, $\phi=\Psi(f)$ and $\psi=\Psi(g)$ are homotopic morphisms of graded $S$-coalgebras $\mathcal{B}_{A} \longrightarrow \mathcal{B}_{B}$. We have the following commutative diagram

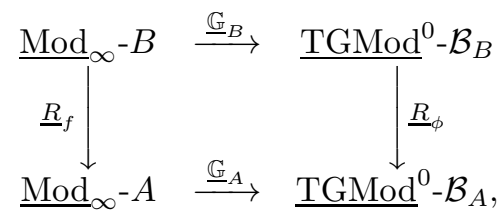

and similarly for the morphisms $g$ and $\psi$. From (5.4), we know there is an isomorphism of functors $\underline{R}_{\phi} \cong \underline{R}_{\psi}$. Denote by $\mathbb{G}_{A}^{\prime}$ a quasi inverse for the equivalence $\mathbb{G}_{A}$. From the isomorphism of functors $\underline{R}_{\phi} \underline{\mathbb{G}}_{B} \cong \underline{R}_{\psi} \underline{\mathbb{G}}_{B}$, we obtain

$$
\underline{R}_{f} \cong \underline{\mathbb{G}}_{A}^{\prime} \underline{\mathbb{G}}_{A} \underline{R}_{f}=\underline{\mathbb{G}}_{A}^{\prime} \underline{R}_{\phi} \underline{\mathbb{G}}_{B} \cong \underline{\mathbb{G}}_{A}^{\prime} \underline{R}_{\psi} \underline{\mathbb{G}}_{B}=\underline{\mathbb{G}}_{A}^{\prime} \underline{\mathbb{G}}_{A} \underline{R}_{g} \cong \underline{R}_{g} .
$$

Acknowledgements. The second author acknowledges the hospitality of Centro de Ciencias Matemáticas, UNAM, during his sabbatical year and the support of CONACyT sabbatical grant 2018-000008-01NACV.

\section{References}

[1] Bautista, R. and Souto, M.J. Categorías Derivadas, Preliminary version, 2016.

[2] Bautista, R., Salmerón, L. and Zuazua, R. Differential Tensor Algebras and their Module Categories. London Math. Soc. Lecture Note Series 362, Cambridge University Press, 2009.

[3] Bondal, A.I. and Kapranov M.M. Enhanced triangulated categories, Math. USSR Sbornik Vol. 70 (1991), No. 1, 93-107.

[4] Brown, K.S. Cohomology of Groups, GTM 87, Springer, 1982.

[5] Happel, D. Triangulated Categories in the Representation Theory of Finite Dimensional Algebras. London Math. Soc. Lecture Note Series 119, Cambridge University Press, 1988.

[6] Keller, B. Introduction to $A_{\infty}$-algebras. Homology, Homotopy and Applications, vol. 3, 1, 2001, pp. 135 .

[7] Lefèvre-Hasegawa, K. Sur les $A_{\infty}$-catégories, Thèse de Doctorat, 2003. 
R. Bautista

Centro de Ciencias Matemáticas

Universidad Nacional Autónoma de México

Morelia, México

raymundo@matmor.unam.mx

E. Pérez

Facultad de Matemáticas

Universidad Autónoma de Yucatán

Mérida, México

jperezt@correo.uady.mx

L. Salmerón

Centro de Ciencias Matemáticas

Universidad Nacional Autónoma de México

Morelia, México

salmeron@matmor.unam.mx 